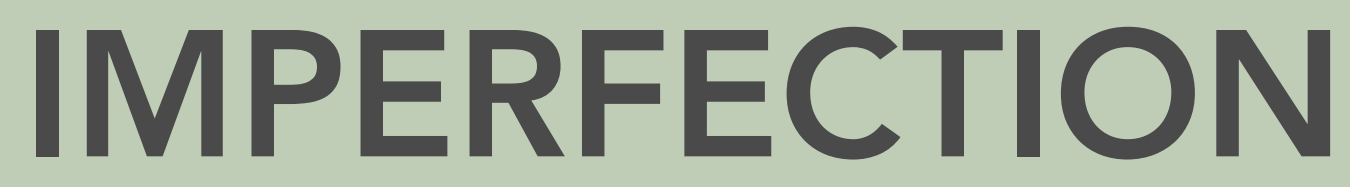
HOLES AND DEFECTS IN SILICON QUANTUM DOTS

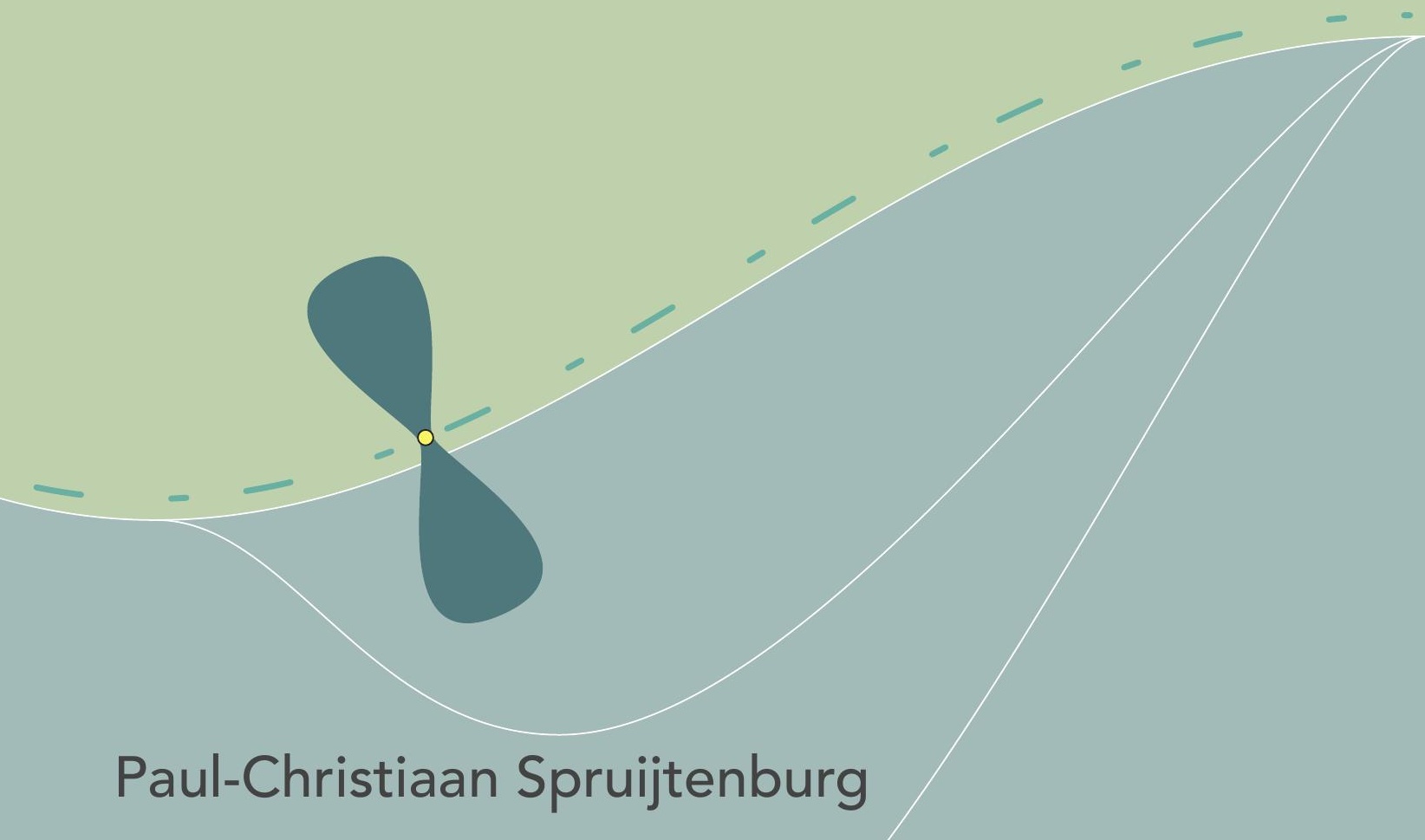





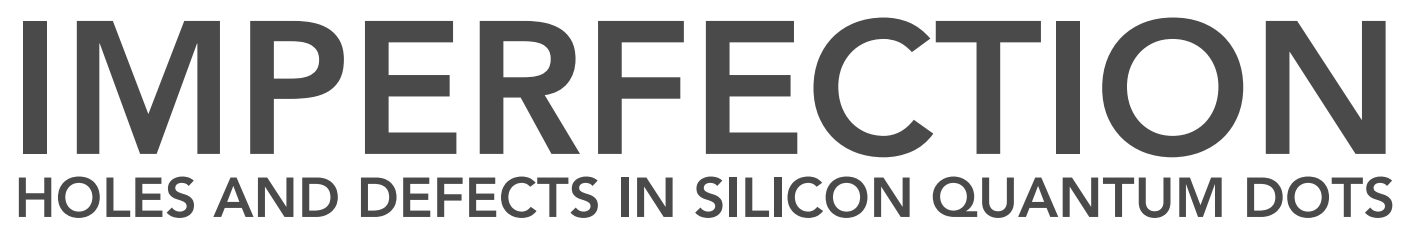

Paul-Christiaan Spruijtenburg 


\section{Ph.D. committee}

\section{Chairman}

prof. dr. P.M.G. Apers University of Twente

Supervisor

prof. dr. ir. W.G. van der Wiel University of Twente

Members
dr. D. Culcer
University of New South Wales
prof. dr. L.M.K. Vandersypen
Delft University of Technology
prof. dr. S.J.G. Lemay
University of Twente
prof. dr. J. Schmitz
University of Twente
dr. ir. F.A. Zwanenburg
University of Twente

\section{Cover}

Design by P.C. Spruijtenburg and Anouk Koster.

\section{Acknowledgements}

The research described in this thesis was performed at the faculty of Electrical Engineering, Mathematics and Computer Science, and the MESA+ Insititute for NanoTechnology at the University of Twente. The main work was financially supported by the Foundation for Fundamental Research on Matter (FOM), which is part of the Netherlands Organization for Scientific Research (NWO).

Imperfection - Holes and defects in silicon quantum dots

Ph.D. thesis, University of Twente, Enschede, The Netherlands

Printed by Gildeprint Drukkerijen

ISBN: 978-90-365-4336-1

DOI: $10.3990 / 1.9789036543361$

(C) P.C. Spruijtenburg, 2017 


\title{
IMPERFECTION - HOLES AND DEFECTS IN SILICON QUANTUM DOTS
}

\author{
DISSERTATION \\ to obtain \\ the degree of doctor at the University of Twente, \\ on the authority of the rector magnificus, \\ prof. dr. T.T.M. Palstra \\ on account of the decision of the graduation committee, \\ to be publicly defended \\ on Wednesday, the $19^{\text {th }}$ of April 2017, at 14:45
}

by

Paul-Christiaan Spruijtenburg

born on the $5^{\text {th }}$ of November 1982

in Groningen, the Netherlands 
Dit proefschrift is goedgekeurd door de promotor: prof. dr. ir. W.G. van der Wiel 


\section{Preface}

The concept of "imperfection" is loaded, and I should be careful not to venture too far into the realm of philosophy, since I am not a philosopher. Even though this little booklet will, with high certainty, result in me obtaining a degree in it.

To talk about imperfection is to talk about perfection. A concept which has come to mean either "that which is complete" (as in subtance) or "that which has attained its purpose". Through these two definitions, a duality rises where even imperfect substances can achieve perfection. For the purpose of the scientific part of this thesis, we will resolve this duality by only considering the former definition and come to talk about crystals and order. At first sight, crystals are a great example of substantive perfection. Completely regular, millions upon millions of atoms show no sign of deviating from their crystal lattice in which they are arranged. In the long run though, even the most regular crystals are not immune to imperfection. Every so often, one can find a single atom slightly out of place, or a wayward atom of a different element has wandered into your carefully prepared lattice. One realizes that in the natural world the concept of "perfection" only holds for a limited time.

As for attaining purpose; well... I remember as a child being fascinated by the glistening pieces in the rocks I found, browsing my little book of naturally occurring crystals and their crystal orientations. Little did I realize how imperfections like impurities were giving these crystals colors. I didn't care about any of that, for the purpose of looking at them they were perfect to me already. I remember years later first being introduced to the concept of the crystal structure in diamonds, and not understanding much of it. But I was fascinated. And so life carries one forward, and here we are today. I would be remiss if I did not thank all the shiny people that were and are in my life; without them I would not be here. Specifically for the last four years, I've met a great deal of people whom I would like to thank for their input, support, and love.

To make a thesis, one first needs to have the space to do the research. The NanoElectronics group gave ample room to do this, and not only physical space. The NE-family is a warm and welcoming bunch. Thank you for making my fourand-a-bit years a very pleasant one.

Of course main instigator in this, and not-at-all-related to Sinterklaas is Wilfred. From our earliest encounters in granting me a great multi-month internship stay 
in Japan, to having me in the group, your impact-factor can be said to be high. You gave great off the cuff suggestions while still managing to always say "hoi" to every single office when you left. ビうもありがとう.

Any thesis is incomplete without a good supervisor. Floris, giving many great tips and input, really fullfiled this role, and in the first year we wrote the fastest written article I've still seen to date. You lured me into the group talking about single-electrons. Speaking of which, I have yet to see one in person. Thank you as well for giving the space and support to carry out my research and voice my ideas. As we understood more and more about the silicon quantum dot system we also understood more about eachother. I also really appreciated the rigour with which you went through all I wrote with a fine-tooth sonic screwdriver and always made the best of a bad sentence. Allons-y!

Home within home was our office, that I had the pleasure of sharing with Elmer and Matthias. With the old "hup-tilly-doooo" in the morning from Elmer, and the occasional "Büro-beben" that Matthias had nothing to do with, it was always a great homecoming. The office-party was ofcourse the pinnacle of our achievement in this, where I finally got to display all that great electronic junk I found somewhere.

Elmer, besides your delightful british morning accent your general happy demeanor was always a good force around. Never too shabby to talk physics, we had some great discussions, beers, the best really bad jokes, and other shenanigans. Pip-pip to you old chap.

Matthias, thank you for welcoming me around the lab when we first got started, and getting me to know the ropes quickly, and for always being a formidable scientific "opponent" to spar against. I really enjoyed the time in our office and outside of it, skiing in the snow during the conferences or when going out for a beer. I got to learn even more excentric german, while you were becoming more and more a true dutch Deichkind. Ksenia, and Matthias, together you made the greatest baking team of NE that no-one knew about. Still the best birthday cakes I've tasted to date!

As far as connections go, the city of Groningen is not a bad one to have. There is something in the spirit of the north that creates connections. So it went with Joost. Of course your EBL- and mad JScript skills deserve mentioning, as they also stood at the cradle of the silicon quantum dot project. From our mildly successfull stint as EBL-inspectors in the "double-bake" greater Britain area, to getting 180 degrees turned around the wrong way in the streets of Japan: thanks for all the laughs. The north remembers.

Beginnings are always hard, and I owe a debt of gratitude to Filipp, who figured out the in-house fabrication of our quantum dots in Twente before I came around. When I had questions about fabrication you were never too shy to explain the exact process steps to me again. The papers we wrote would not have been possible without you.

When my $\mathrm{PhD}$ was at its most discouraging, and I was alone in the project 
after Filipp left, Sergey swooped in and saved the day. Your indomitable energy and spirit not only made samples and wafers but infused me with new ideas and a hope that I might actually finish this darn thing. Never too taken aback by me having a crazy idea, we schemed and plotted. It made me realize that indeed, science does not happen in a vacuum. Don't ever lose that enthusiasm, it is your biggest strength.

In a group there are also always the people that have been around longer than you have, and seen the countless PhDs, postdocs, and other students come and go. I can tell you, it's in any case also not easy from this side to leave.

Jonny, somehow I've never seen a more calm and collected technician. I really enjoyed the atmosphere that you brought to the group and talking to you about everything and nothing. Martin, thank you for all the fun components you ordered or had lying around that I could make crazy things with. The office party would have been a different place without it. Thijs, thank you for a lot of things around the lab, but most of all thank you for getting me that laptop of that fruit-related brand. I know it was not easy. But know I could not have finished my thesis without it! One could say a great administrative help is seen by not being seen. Thank you Karen therefore, for your sunny personality and doing all the countless paperwork I never did see. Michel, unfortunately we only got the chance to collaborate on a 7-minute voronoi-doohickey. Even so, I really enjoyed the discussions at the coffee table and your great insights into physics.

On the fabrication side I would like to thank the entire cleanroom staff, without which all the beautiful nano would not be possible. In particular though, I would like to thanks Hans, who somehow always managed to beat your expectations. The zesty spirit you brought with you into the cleanroom and outside of it was much appreciated. Ik heb dan altijd met veel plezier met je gebakkeleid over vanalles en nogwat. En dat ondanks dat ik een ouwe hippie ben. Het ga je goed. I would also like to thank Rico, for his dilligence and going beyond the call of duty and measuring things just in time to make it to this thesis. I don't think there is a better way to make TEM measurements, with Rico's calm demeanor and classical music in the background. I will most definitely try and replicate this most relaxing environment should I ever get the opportunity. Tom Aarnink also deserves a word of gratitude for building the great ALD-clusters in the cleanroom, and for always being open and not being afraid to try some of those new processes we came up with.

As students go, a good quality to find is the ability to ask questions. Abla never found a reason to stop asking them, so I would like to mention her. I would also like to thank the other students that made valuable measurements and gave new insights: Ton, Bram, Max, and Yizhen.

Outside the confines of the academic world I spent most of my time roaming around Enschede for a good 10-odd years and a little more. It was a great experience which only got better with time. As excursions from the city took me to some beautiful festivals, big cities and who knows where else, I always met the nicest 
people. A red thread (as they say) running through this time was also art. I think everyone could stand with a little more art in their lives, and the academic world could stand to learn from these processess just a little more. So, I don't know how I would have even started writing my thesis if I didn't get to call the cafe at the Robson my atelier for the evenings. So I would like to thank Wouter and Marloes, for turning the whole Robson ex-pyjama factory into an atelier space. In doing so giving so many people a second home, building a community, with room to grow. In this vein, I would like also to thank Miguel for the coffees there, and for the blue(s) paintings to stare at and get inspiration during the nights.

Another big part of my life in the past years was bikes! From starting to ride fixed-gear bikes with Rob, riding the streets every "fixed-friday" to playing bike polo. We made the evolution, rode the dream. My bike and polo-homies and allround super friends: Robbert, Mark, Laura, Kay, Karen, Philip, Natalia, Anna, Franz, Matthijs, Dario. For taking us places to play weird tournaments, and building the awesome court in the polaroid. Such a great bunch of friends to talk to and have fun games with. I'm surprised we didn't break more legs!

I would count good friends as people that, even while losing track of time, you would find again, finding no time has actually passed. Marco, thank you for your timeless friendship, the food, the quilombo, the polo, the granada, the girlfriend, talking theses, talking future, plumbuses, where do I even end this list?

Finally, I get to thank my parents in writing. Mama, Papa, thank you for always providing a safe environment and for giving me the opportunity to look at the glistening pieces on the shores, valleys, and countless mountains. Thank you, for listening and supporting me through my stints of youthful exhuberance. Just the three of us.

To all the perfectly imperfect,

Paul-Christiaan Spruijtenburg

March 2017 



\section{Contents}

1 Introduction 1

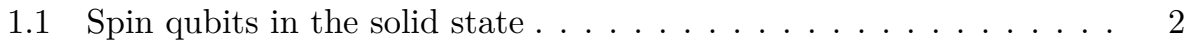

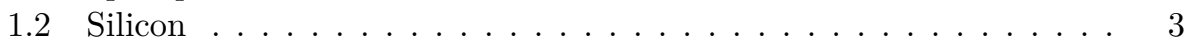

L.3 Holes in silicon . . . . . . . . . . . . . . . . . . . . . . 3

1.4 Imperfection. . . . . . . . . . . . . . . . . . . . 4

$\square .5$ Outlind . . . . . . . . . . . . . . . . . 4

$\begin{array}{lllll}2 & \text { Theoretical background } & 7\end{array}$

2.1 Quantum dots . . . . . . . . . . . . . . . . . . 7

22 Holes in silicon . . . . . . . . . . . . . . . . . . . . 12

2.3 Defects . . . . . . . . . . . . . . . . . . . 15

3 Quantum dot heterostructures 19

3.1 The electrostatically defined quantum dot heterostructure . . . . . 20

3.2 Layer synthesis techniques $\ldots \ldots \ldots \ldots \ldots \ldots \ldots$

3.3 Defects in quantum dot heterostructures $\ldots \ldots \ldots \ldots \ldots$

3.4 The annealing process $\ldots \ldots \ldots \ldots \ldots \ldots \ldots \ldots$

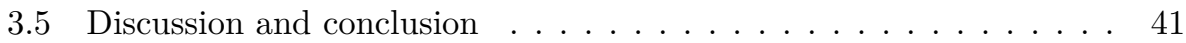

4 Single-hole tunneling through a two-dimensional hole gas in in$\begin{array}{ll}\text { trinsic silicon } & 45\end{array}$

4.1 Fabrication . . . . . . . . . . . . . . . . 46

42 Holes . . . . . . . . . . . . . . . . . . . . . . . 47

4.3 Discussion and conclusion . . . . . . . . . . . . . . 50

5 Passivation and characterization of charge defects in ambipolar silicon quantum dots 53

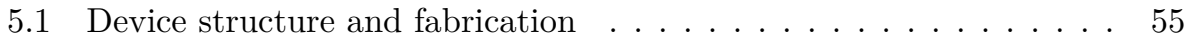

5.2 properties of $\mathrm{Al}_{2} \mathrm{O}_{3}$ grown by atomic layer deposition $\ldots \ldots \ldots . \quad \ldots 7$

5.3 Quantum dot formation before and after annealing . . . . . . . 57

5.4 Isolated charge defects $\ldots \ldots \ldots \ldots \ldots \ldots \ldots$

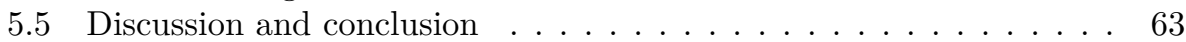


6 g-tensor anisotropy in a planar silicon hole quantum dot $\quad 65$

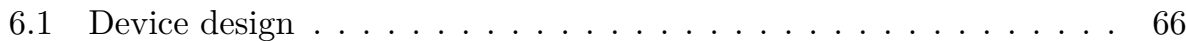

6.2 Initial characterization and dot formation . . . . . . . . . . 67

6.3 Fitting procedures $\ldots \ldots \ldots \ldots \ldots \ldots \ldots \ldots$

6.4 Magnetospectroscopy $\ldots \ldots \ldots \ldots \ldots \ldots \ldots$

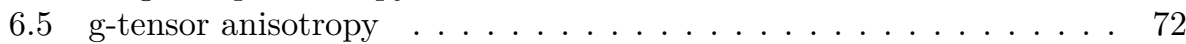

6.6 Discussion and conclusion . . . . . . . . . . . . . 78

7 Single-layer depletion-mode hole quantum dots in intrinsic silicon 83

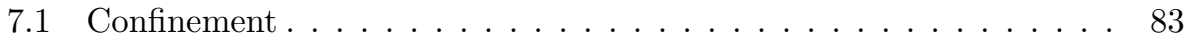

$7.2 \quad$ Gate design $\ldots \ldots \ldots \ldots \ldots \ldots \ldots \ldots \ldots \ldots$

7.3 Tuning between a single and double quantum-dot . . . . . . . . . 85

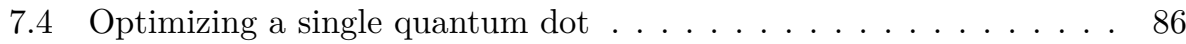

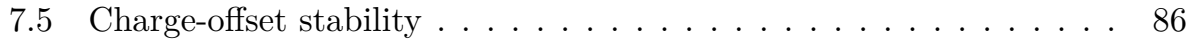

7.6 Discussion and conclusion . . . . . . . . . . . . . . . 89

8 Conclusions and outlook 93

8.1 Hole quantum dots . . . . . . . . . . . . . . . . . . . . . . 93

8.2 Heterostructures $\ldots \ldots \ldots \ldots \ldots \ldots \ldots$. . . . . . . . . . 94

8.3 Depletion-mode quantum dots . . . . . . . . . . . . . . . . 95

8.4 Single-electron/single-hole transistors $\ldots \ldots \ldots \ldots \ldots . \ldots 96$

8.5 Final thoughts $\ldots \ldots \ldots \ldots \ldots \ldots \ldots \ldots \ldots$

$\begin{array}{ll}\text { References } & 97\end{array}$

\begin{tabular}{ll}
\hline Appendices & 111
\end{tabular}

A Design of high-frequency transmission lines for low electron $\begin{array}{ll}\text { temperature } & 113\end{array}$

\begin{tabular}{ll}
\hline B g-tensor anisotropy & 117
\end{tabular}

B.1 simple Gaussian fitting method . . . . . . . . . . . . . . . . . . 117

B.2 parametric fitting method . . . . . . . . . . . . . . . . . 117

B.3 Uncorrected anisotropy data accross principal planes . . . . . . . . 118

$\begin{array}{ll}\text { Summary } & 121\end{array}$

$\begin{array}{ll}\text { Samenvatting } & 125\end{array}$ 



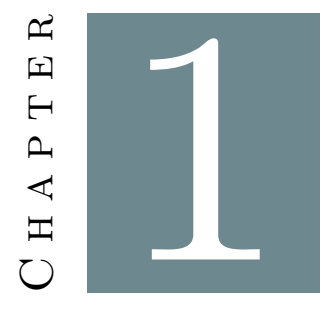

\section{Introduction}

There is plenty of room at the bottom. But where is the bottom, and how much room is there, exactly?

Feynman's lecture $(1959)^{\boxplus}$ articulated and drew a logical conclusion to a process which was already underway at the time: the manipulation and control of materials at ever decreasing scales. He postulated that there is no physical reason, why we cannot control and exploit materials at their smallest scales, the scale of atoms, where quantum mechanics holds sway. This is - and probably will be for several hundred years more - our bottom.

It is exactly these fundamental physical laws which the semiconductor industry has run into, while inching the size of transistors ever closer to the atomic scale. At these length scales, the tunneling of electrons through thin dielectrics cause gate leakages, and has already forced chipmakers to adopt high-k dielectrics in order to maintain gateability of transistors and keep leakage currents manageable.

But instead of fighting these laws, why not embrace these quantum mechanical effects? The idea of basing computation on quantum mechanics was already proposed as far back as 1969 by Steven Wiesner as a way to better accomplish cryptologic tasks. ${ }^{\text {[I }}$ But it was Feynman, in 1982, who was among the first to put forth a quantum system which could be used to do computations. ${ }^{[1}$ The first universal quantum Turing machine followed suit and was proposed by Deutsch in 1985, and paved the way for the quantum circuit model. ${ }^{4}$

In the quantum circuit model, information is encoded in a quantum bit or qubit, which can be in a linear superposition of its basis states: $\psi=\alpha|0\rangle+\beta|1\rangle$ (with coefficients $\alpha$ and $\beta$ complex numbers). The information is encoded in the coeffi- 
cients $\alpha$ and $\beta$, which is why $\mathrm{N}$ qubits can encode $2^{N}$ bits of classical information - that's a lot of room.

The information stored in the qubits can then be manipulated using quantum algorithms, the most famous of which are Grover's algorithm (1996), and Shor's algorithm (1994). ${ }^{\text {. }}$ Shor's algorithm is capable of number factorization in polynomial time. This tremendous "speed-up" as compared to the fastest known classical solution is one of the prime factors which lead to the interest in quantum computation.

Other paradigms to utilize quantum resources to speed-up computation have also emerged, such as adiabatic algorithms, Topological-Quantum-Field-Theory (TQFT) Algorithms, and measurement-based algorithms. ${ }^{\square}$ It is interesting to note that it is still not entirely clear what exactly constitutes the resource a quantum algorithm uses to speed-up certain classes of computational problems.

Thankfully, the development of a quantum computer need not be solely contingent on these well-known algorithms. The use of quantum computers in e.g. quantum chemistry would greatly improve the accuracy and speed in simulations of molecules or other intrinsically quantum mechanical systems. A working quantum computer would also allow foundational explorations of quantum mechanics, and one could ask questions such as: What actually is the quantum resource in use in a quantum computer, or, can we scale a quantum state indefinitely? Where does the classical world begin and the quantum world end, and most importantly, can we make a macroscopic cat state? ${ }^{\mathbb{W}}$ Whether or not a real quantum computer will ever be successfully built, or have the "magical" properties we hope for it - in the process building of it, we inevitably learn more about the nature of quantum mechanics and the limitations of man.

\subsection{Spin qubits in the solid state}

Given the prospects of quantum computation, work began on creating these quantum bits. The first experiments were performed in cold-ion traps, ${ }^{\underline{Q}}$ and liquid-state nuclear magnetic resonance. ${ }^{\text {II }}$

It is one thing to make a one or two-qubit system, but the success of a future quantum computer hinges on reliably and controllably scaling up from several qubits to several hundred qubits, or even millions in a quantum error correction scheme such as surface code. Even with error correction the requirements placed on these qubits is on the order of $99 \%$ readout fidelity, and trades off the number of qubits against a tolerance for errors. ${ }^{\text {WI }}$

So, our qubits have to be reproducible, with a long-lived spin, and we need to have a larger number of them close together. The solid state lends itself well to this, as the CMOS industry has already solved the scaling-up problem to a large

\footnotetext{
${ }^{1}$ Although Hawking seems to object to the notion of cat states ${ }^{\sqrt{t}}$ and is noted for stating: "When I hear of Schrödinger's cat, I reach for my pistol"
} 
degree. In the context of quantum computing this means that devices can be scaled up, reliably and even have the option to integrate classical control- and readout-logic close by.

A concrete proposal was done by Loss and DiVincenzo [1:2], who suggested quantum logic gates could perform operations on electron spins in neighbouring quantum dots (QD).

Following this proposal a whole zoo of experiments in GaAs was done, with the first shell-filling of a quantum dot reported by Tarucha et al. [13]. Spinreadout, a crucial part for spin-based quantum computation, was demonstrated by Elzerman et al. [14] using a nearby quantum point contact. The coherent control and manipulation of electron spins followed soon after.

\subsection{Silicon}

One of the problems of embedding spin qubits in III-V materials, such as GaAs, is the abundance of nuclear isotopes with a non-zero nuclear spin. The spinqubits couple to this nuclear spin-bath, and as a result suffer from decoherence. The reduction of this effect is the main motivation for the introduction of $\mathrm{Si}$ as a host material. Silicon already has a relatively low concentration of nonzero nuclear spin isotopes, and can be isotopically purified to ${ }^{28} \mathrm{Si}$. The lack of spin decoherence mechanisms for states inside the bandgap has led to the term "semiconductor vacuum". "Single atoms lend themselves extremely well to this environment, as their levels are deep inside the Si bandgap and thus strongly decouple from the environment. This was shown in spin-resonance experiments on a single phosphorus atom implanted in $\mathrm{Si}$. 18

The first planar quantum dots based on Si were demonstrated in Si/SiGe quantum wells ${ }^{\square 9}$ and in electrostatically-defined quantum dots ${ }^{\sqrt{21}}$ Follow-up work showed it was possible to create double quantum dots, 2,221 and to coherently drive Rabi oscillations and implement a two-qubit logic gate with $99.6 \%$ control fidelity. $2.3,24$ This architecture is essentially an extremely small MOSFET and therefore eminently compatible with CMOS processes.

\subsection{Holes in silicon}

Thus far, all quantum dot systems discussed were based on the spins of electrons. Holes, the counterparts of electrons in the valence band, have properties that make them an interesting candidate for quantum computation. Hole quantum dots were first demonstrated in Si nanowires, ${ }^{[25}$ with later reaching the single-hole regime. ${ }^{[26}$ More recently, Pauli-spin blockade ${ }^{[\pi]}$ and a CMOS compatible hole quantum dot were shown. $\frac{28}{}$ 
Holes have p-like orbitals which exhibit only a small overlap with the nucleus. Therefore the contact hyperfine interaction - the nuclear spin that the hole spin

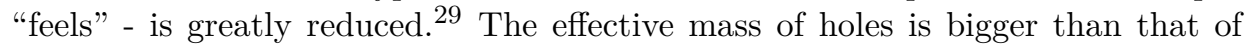
electrons, and expected to behave in a non-trivial manner as a function of strain. Perhaps the most interesting aspect of holes is that whereas the spin-orbit coupling for electrons in $\mathrm{Si}$ is effectively zero, the behaviour of holes is deeply affected by it. The spin-orientation of holes is expected to be preferentially oriented in $2 \mathrm{D}$ quantum wells ${ }^{31}$ due to spin-orbit coupling, and the coupling to magnetic field expected to be very dependent on the shape and size of the quantum dot. $\underline{\text { B. }}$

These properties make holes as the carrier of spin in a quantum dot a very interesting system, both technologically and fundamentally.

\subsection{Imperfection}

So far, we have assumed that these quantum dots live in perfectly ordered model systems, and are decoupled from their environment. The reality is of course somewhat less ideal.

Known sources for decoherence that relate to the environment are e.g. chargeand spin-noise, $\stackrel{3.3}{ }$ and glassy two-level systems that cause long-term fluctuations of the electric-field. ${ }^{[34}$

We see that the properties of the host environment become important as the coherence of the quantum states is affected. No material is ever perfect, and imperfections - defects - remain, since they give the system the chance to lower its free energy. Defects are not always unwanted however, and sometimes even induced intentionally like with impurity doping of semiconductors, or with nitrogen-vacancy (NV) centers in diamond. Every material, or every combination of materials, has its own specific type of defects. For Si based platforms the most technologically relevant defects have turned out to form at the $\mathrm{Si} / \mathrm{SiO}_{2}$ interface and are called $\mathrm{P}_{\mathrm{b}}$ centers. ${ }^{[5,, 36}$

The control, influence, and/or mitigation of these imperfections relate to the performance and feasibility of a scaled-up quantum computer and cannot be seen separately.

\subsection{Outline}

This thesis will focus on the transport and fundamental properties of holes in silicon quantum dots and discuss the role of defects in the formation of quantum dots.

We will start by explaining the theoretical concepts governing quantum dot transport measurements and point defects in Chapter ㄱ. 
Chapter [3 will attempt to give an in-depth picture of the heterostructures that planar quantum dots are based on. It will relate the properties of heterostructures to the requirements for successful quantum dot formation.

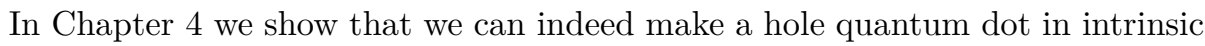
silicon, by demonstrating single-hole tunneling through an electrostatically defined quantum dot.

Having demonstrated single-hole tunneling, we set out to improve these results and investigate defects in our heterostructure. We do this in Chapter $\mathbf{0}$, where we attempt to passivate defects using $\mathrm{Al}_{2} \mathrm{O}_{3}$ grown by atomic-layer deposition. The hydrogen in this oxide has passivating properties, and we investigate remaining charge defects in our heterostructure.

Chapter $\mathbf{6}$ focuses on the behaviour of holes as carriers of spin, as related to their predicted spin-orientation in a semi-2D quantum well. We do this by studying the Zeeman splitting in a magnetic field capable of rotating $360^{\circ}$ over all 3 degrees of freedom.

In Chapter $\mathbf{\square}$ we will discuss a proof-of-principle of a single-layer depletion-mode hole quantum dot, made possible by negative fixed charge in ALD-grown $\mathrm{Al}_{2} \mathrm{O}_{3}$.

In the final chapter (Chapter 8 ) we discuss the main results and place them in the context of the quantum computing field. Here we will also look at possible avenues for future scientific exploration. 



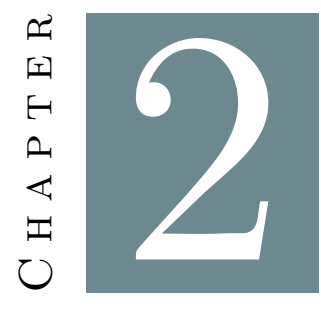

\section{Theoretical background}

In this chapter we will consider the most important theoretical aspects that underlie this work. First, we will describe quantum dots and the criteria necessary to observe single-electron tunneling in quantum dots. Then, we will focus on the constant-interaction model which describes charge stability on quantum dots. We will discuss the properties of holes in silicon, and finally will describe the theoretical background of defects at the $\mathrm{Si} / \mathrm{SiO}_{2}$ interface.

\subsection{Quantum dots}

Quantum dots are artificially constructed objects in the solid state, confining a well-defined number of charge carriers to a region small enough, such that the quantum energy levels are no longer degenerate at the energy scales under consideration. These discrete energy states in many ways resemble the energy states associated with atoms, and even exhibit orbital shell filling, which is why quantum dots are also colloquially referred to as artificial atoms.

\subsubsection{Single-electron tunneling}

In this work quantum dots are created in between two tunnel junctions, and measured by observing the transport behaviour of single electrons (or holes) through the discrete energy states of the quantum dot.

To understand the criteria for the observation of single-electron tunneling, first 


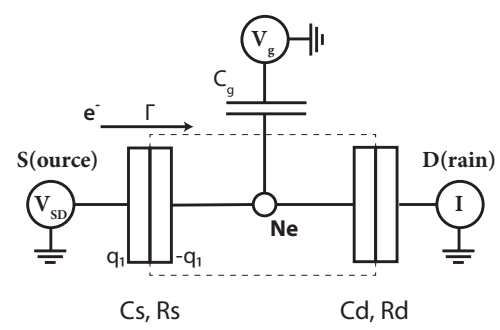

Figure 2.1: Quantum dot between two tunneling junctions. Tunneling rate $\Gamma$, source-drain bias $V_{S D}$, current $I$, charge on the dot $N e$, tunnel junctions with equivalent capacitance $C_{s, d}$ and $R_{s, d}$

consider that the energy stored on a capacitor, $E=q^{2} / 2 C$, is not quantized but consists of the polarization charges $q$ generated by displacing the electron gas with respect to positive lattice charge. ${ }^{[37}$ The fractional polarization charge on a capacitor can be continuously increased, up to the point where the Coulomb repulsion is overcome and it is energetically favourable for an electron to tunnel onto the dot. Before tunneling can occur, the system is in Coulomb blockade. The energy needed to overcome Coulomb blockade and add an electron to the dot is defined as the charging energy $E_{C}$. To avoid multiple electrons tunneling at the same time, the thermal energy should be negligible compared to the charging energy: $E_{C} \gg k_{B} T$. The second consideration is that the uncertainty in energy $d E$ is determined by the Heisenberg uncertainty principle: $d E d t=\left(e^{2} / C\right) R C>$ $h$. Therefore, the tunneling resistance should be higher than the conductance quantum $R \gg h / e^{2}$ or, written in terms of charging energy $E_{C} \gg h \Gamma$

The charging energy $E_{C}=e^{2} / C$ of the quantum dot should be larger than the thermal energy $k_{B} T$ and the tunnel coupling $h \Gamma: E_{C} \gg k_{B} T, h \Gamma$. Here $C$ being the sum of all capacitances to the quantum dot, $k_{B}$ the Boltzmann constant, $T$ the temperature, $h$ the Planck constant, and $\Gamma$ the tunneling rate.

The second criterion concerns the word "quantum" in quantum dot and requires the quantization of the wavefunctions of the carriers, completely analogous to the perennial example of the quantum mechanical particle-in-a-box. This is achieved when the carriers are confined to a region $L$ small enough, such that the eigenstate energies $E_{n}=(n \kappa \pi)^{2} /\left(m^{*} L^{2}\right)$ are separated by at least tunnel broadening $h \Gamma$ and thermal broadening $k_{B} T$.

The orbital level spacing on the quantum dot should be larger than the thermal energy and tunnel broadening, $\Delta E \gg h \Gamma, k_{B} T$. 


\subsubsection{Constant-interaction model}

The constant-interaction model describes the energy spectrum of a quantum dot as a function of the number of electrons on the dot.

(a)

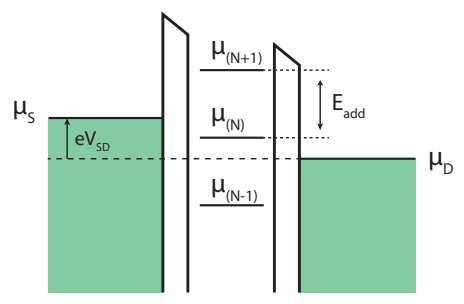

(b)

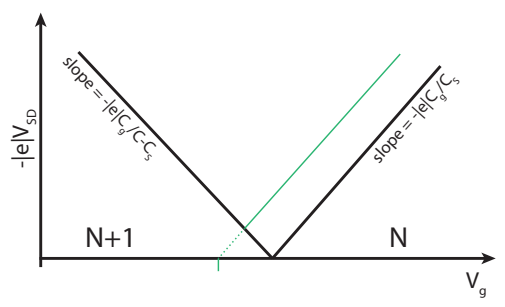

Figure 2.2: Constant-interaction model (a) Transport occurs when the electrochemical potential of the dot is in the bias window: $\mu_{s}<\mu(N)<\mu_{d}+e V_{S D}$. (b) Stability diagram for holes, relating the voltage on the gate $V_{g}$ to the transport properties and Coulomb blockaded regions of constant electron occupation N. The red line indicates transport through an orbital excited state.

The model makes two assumptions. The first assumption is that the Coulomb interactions among electrons in the dot, and of the electrons in the dot to the environment can be parametrized by the sum of all capacitances to the dot. $C_{\Sigma}=$ $C=C_{s}+C_{d}+C_{g}$. The second assumption is that the single-particle energy level spacings are not dependent on the occupation of the $\operatorname{dot}^{38}$

This means that the total energy of the dot can be described as the sum of the energy stored in electrostatic- and quantum-confinement:

$$
U(N)=\frac{\left(-|e|\left(N-N_{0}\right)+C_{g} V_{g}+C_{s} V_{s}+C_{d} V_{d}\right)^{2}}{2 C}+\sum_{n=1}^{N} E_{n}
$$

A convenient model to describe transport through the quantum dot can be obtained by switching to a representation in electrochemical potential $\mu(N)$. The electrochemical potential of a body with a fixed number of electrons $\mathrm{N}$ is defined in the canonical ensemble $\mathrm{e}^{\mathbf{3 9}}$ as:

$$
\begin{aligned}
\mu(N) \equiv & U(N)-U(N-1) \\
= & \left(N-N_{0}-1 / 2\right) E_{C} \\
& -\frac{E_{C}}{|e|}\left(C_{s} V_{s}+C_{d} V_{d}+C_{g} V_{g}\right)+E n
\end{aligned}
$$

The second term of this equation implies that the electrochemical levels of the states can be linearly and continuously shifted by applying a voltage on any of the terminals. 
The energy needed to add an electron to the dot is $E_{a d d}=\mu(N+1)-\mu(N)=$ $E_{C}+\Delta E$, and is comprised of the electrostatic part and the orbital level spacing $\Delta E$. When adding a second electron to the same spin-degenerate level the contribution of $\Delta E$ vanishes.

\section{Transport and tunneling features for a single quantum dot}

Transport through the dot is now easily understood through the electrochemical potential picture in the constant-interaction model. We draw this schematically in Fig. [2.2. The gate voltage $V_{g}$ controls the electrochemical potential ladder on the dot, and the source-drain bias $V_{S D}$ the level of the electrochemical potential in the source $\mu_{S}$. When a level on the quantum dot is between the electrochemical potential levels of the source $\mu_{s}$ and the drain $\mu_{d}$, transport through the dot can occur. If none of the ground-state levels are in the bias window, the dot is in Coulomb blockade.

The interplay of the voltages applied through the terminals can be represented in a stability diagram. This stability diagram is typically a function of the sourcedrain bias $V_{S D}$ and the gate voltage $V_{g}$. Regions where the charge is constant, because the electrons do not have enough energy to overcome Coulomb repulsion, and no electrons are tunneling on or off of the quantum dot, are seen as parallelograms called "Coulomb diamonds". When a level enter the bias window $|e| V_{S D}$, single-electron tunneling takes place. Fig. $2.2 b$ schematically shows the resonant tunneling features in the stability diagram for holes, which is (because of the positive charge of holes) the point-symmetric mirror image of the electron stability diagram. The Coulomb diamond edges are when the ground state is resonant with either the source or drain and are given by $-|e| C_{g} /\left(C-C_{s}\right)$ and $-|e| C_{g} / C_{s}$, respectively. When an orbital excited state with energy $\Delta E$ is resonant with either source or drain, this is seen as a line of increased conductance, parallel to one of the Coulomb diamond edges.

\subsubsection{Quantum dots in MOSFETs}

Quantum dots made in planar metal-oxide-semiconductor field-effect transistor (MOSFET) structures are for almost all intents equal to traditional MOSFET structures. The classic MOSFET consists of a semiconductor, an insulator, and a metal gate (Fig. [2.31).

In this structure, the metal and the semiconductor material have a different work function and therefore bringing these two materials into thermal equilibrium requires compensation charges in order to equilibriate the fermi level. The amount of charge necessary for this is determined by the workfunctions of the metal $\Phi_{m}$ and the semiconductor $\Phi_{s}$. The local compensation charges lead to what is called band bending. The band-structure of the semiconductor has moved up or down in 
terms of energy close to the insulator. Thus, choosing different materials for the metal gate leads to different band bending.

(a)

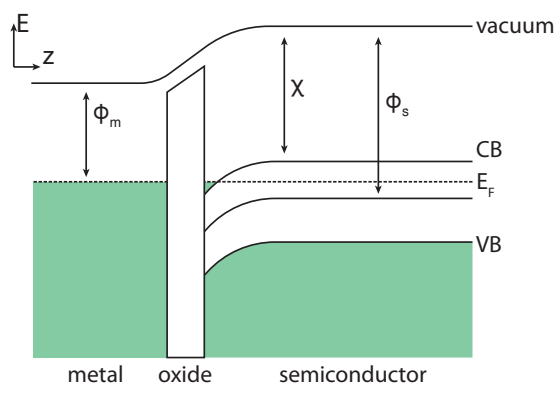

(b)

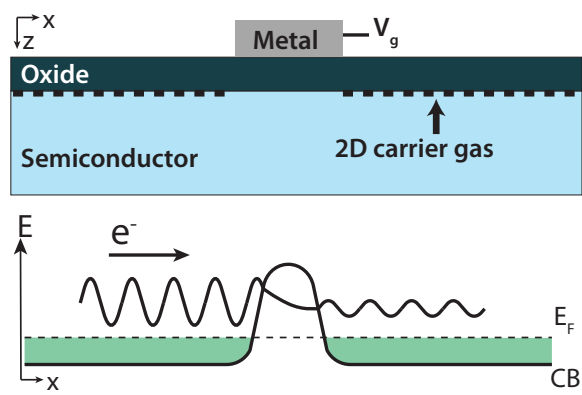

Figure 2.3: Band bending in a MOSFET. (a) Band bending after Fermi level $E_{F}$ equilibriation. The conduction band close to the oxide-semiconductor interface dips below the Fermi level, and thus electron states (green) are available for transport. [b] Creating a tunnel barrier by local band modulation by applying voltage $V_{g}$ to an electrode. Here the 2DEG is assumed to be intrinsically present in the semiconductor and locally depleted by a chosen potential on the metal gate (bottom panel).

A convenient quantity to compare band-bending is the flatband voltage. This is defined as the voltage where band-bending is reduced to zero, and in the case of an intrinsic semiconductor given by:

$$
V_{F B}=\left(\Phi_{m}-\Phi_{s}\right) / e
$$

with $e$ the elementary charge.

As an example, the two metals used in this work have the work functions $\Phi_{m}(\mathrm{Al})=4.1 \mathrm{eV}, \Phi_{m}(\mathrm{Pd}) \approx 5.4 \mathrm{eV}$. For our semiconductor $\mathrm{Si}$, the workfunction is assumed as $\Phi_{S i}=\chi+E_{g} / 2=4.15+0.56=4.71 \mathrm{eV}$. Here the electron affinity $\chi$ is the energy difference between the conduction band and the vacuum level and $E_{g}$ is the band gap. This leads to the flatband voltages $V_{F B}(\mathrm{Al})=-0.61 \mathrm{~V}$ and $V_{F B}(\mathrm{Pd})=0.69 \mathrm{~V}$. Since the threshold voltage of the MOSFET is given by $V_{t h}=V_{F B}+2 \phi_{F}$, with $\phi_{F}$ the surface potential, the threshold voltage shifts accordingly with a change in material chosen for the gate metal. Any changes in the intrinsic Fermi level of the semiconductor (due to e.g. charged defects or doping) also influences the band bending, since the work function of the semiconductor is defined by the electron affinity $\chi$.

The creation of a quantum dot requires two tunnel junctions, which are easily created in MOSFET structures by locally controlling the potential using electrodes. When the potential pulls the conduction band far enough above the Fermi level, a potential barrier is created for the electrons (see Fig. 22.3b). With the correct width and height of the potential barrier, electrons can tunnel through the barrier. Simply speaking, the only remaining challenge is to pattern the elec- 
trodes close enough together to satisfy the requirement for size quantization of the wavefunctions on the quantum dot.

\subsection{Holes in silicon}

The use of silicon as a host material for quantum dots has been spurred by the promise of creating a nuclear-spin free environment by isotopic purification to ${ }^{28} \mathrm{Si}$, which would essentially create, for states inside the bandgap, a semiconductor vacuum. The promise of holes as carriers of spin, as opposed to electrons, is for several reasons. The wavefunction of holes in the valence-band is p-type, and therefore the overlap of the wavefunction with the Si host nuclei is greatly reduced through a reduced contact hyperfine interaction, theoretically increasing the spin-lifetimes when used in quantum computation even further. The p-type wavefunction also implies that the orbital angular momentum is $l=1$, resulting in a finite spin-orbit coupling. This enables electron-dipole spin resonance (EDSR) and possibly g-tensor modulation, which we will touch on in Chapter 6l. Finally, holes do not have valley-degeneracy, which means there are less decay channels available.

In this thesis, we will focus almost exclusively on the behaviour of holes inside this semiconductor.

Holes are positive-charge, positive-mass quasiparticles, that come about by the absence of an electron in a nearly-full valence band. That we may switch from a description where charge is carried by electrons, to one where it is carried by holes, stems from three observations.

1. A completely filled band carries no current, which is expressed thusly:

$$
0=\int_{\text {zone }} \boldsymbol{\nu}(k) d \boldsymbol{k}=\int_{\text {unoccupied }} \boldsymbol{v}(k) d \boldsymbol{k}+\int_{\text {occupied }} \boldsymbol{v}(k) d \boldsymbol{k}
$$

where zone indicates the first brillouin-zone, $\boldsymbol{v}$ the group velocity, and $\boldsymbol{k}$ the wave vector of the electrons.

It can be seen that an integral over all the occupied states (that normally is proportional to the current density) can be expressed as negative the contribution of the integral over all unoccupied states. The contribution of the absence of an electron can therefore be expressed as a positively charged particle which moves in the same direction as when the state were occupied by an electron.

2. The motion of all points in the band are completely determined in time by their momenta $\boldsymbol{k}$ and position $\boldsymbol{r}$ by the semiclassical equations of motion. Therefore the hole (or absence of electron) behaves exactly as as if there was an electron present. This is counter the well-known analogy of empty seat in the concert hall or of cars stuck in traffic. 
3. The electrons in the valence band, when subject to a force $\boldsymbol{F}$, undergo an acceleration $\boldsymbol{a}$ determined by the effective mass tensor $\boldsymbol{M}^{-1}(k)$ through Newton's second law $\boldsymbol{F}=m \cdot \boldsymbol{a}$. Assuming a parabolic dispersion, the effective mass at the top of the valence band is $\boldsymbol{M}^{-1}=\kappa^{-2} \partial \epsilon^{2} / \partial k^{2}<0$. The acceleration is thus oriented in the opposite direction from the applied force. As if salmon, the electrons at the top of the valence band move upstream by virtue of the group velocity of the wavefunctions.

Therefore, the absence of electron at the top of the valence band would move the same way as a positive-mass, positively-charged particle at the bottom of the conduction band: a hole.

\subsubsection{Holes and the band structure of silicon}

Silicon, in its most common form, crystallizes in a face-centered diamond-cubic structure. The crystal structure is not inversion asymmetric in the bulk. Silicon shares four of its electrons in tetrahedrally oriented covalent bonds with its neighbours in $\mathrm{sp}^{3}$-hybridized orbitals. The bandgap of silicon is indirect, with a band-gap energy of $E_{g} \approx 1.11 \mathrm{eV}$ at room temperature.

(a)

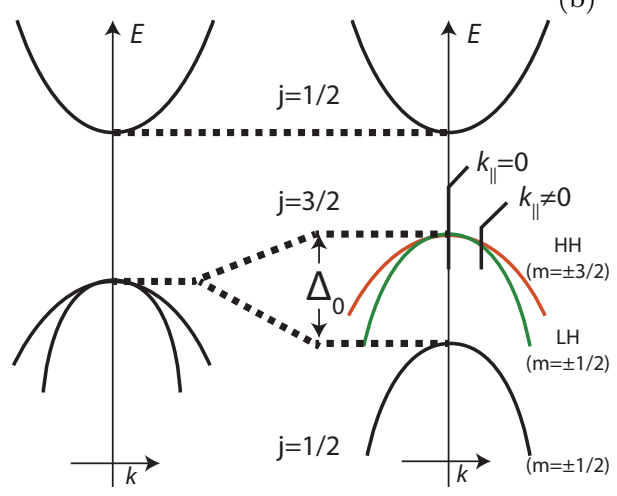

(b)

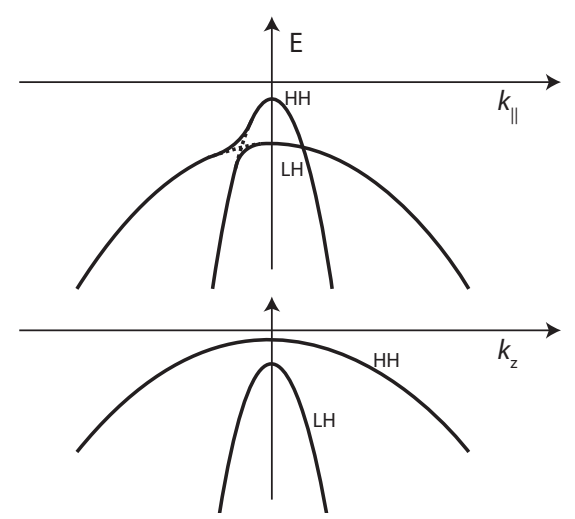

Figure 2.4: Schematic overview of the band structure of Si. The indirect bandgap is not shown. (a) (left) the band-structure as it would be without SOC. (right) With SOC, the states with $j=1 / 2$ are located in the split-off band and are separated by $\Delta_{0}$ from $j=3 / 2$. The light-hole and heavy-hole states with $m_{j}= \pm 1 / 2$, and $m_{j}= \pm 3 / 2$ are visible. (b) Dispersion of the valence band in a semi-2D quantum well in (top) the $k_{\|}$direction showing the HH-LH splitting as a consequence of size quantization, with avoided crossings indicated by the dashed lines. (bottom) the dispersion in the $k_{z}$ direction is HH-LH split without any avoided crossings.

The band minimum for electrons in the conduction band is located at $k=0.85 k_{0}$ in the [001] crystal direction, with $k_{0}$ being the edge of the zone boundary. The 
non-zero momentum leads to six-fold degenerate "valleys". For holes the maximum of the valence band is located at $k=0$.

\section{The valence band in bulk}

The spin-orbit (SO) interaction plays an important role in semiconductor physics. It is responsible for coupling the spin and orbital degrees of freedom. The quantum state is no longer properly defined by either of the quantum numbers separately and the proper quantum number is thus the total angular momentum $j=l+s$.

This has the following effects for the orbital motion of electrons in semiconductors. In the tight-binding picture, the electrons in the conduction band are best described by s-like atomic orbitals (orbital angular momentum $l=0$ ), and the total angular momentum $j$ for electrons in the conduction band $(\mathrm{CB})$ is simply the spin angular momentum $j=s=1 / 2$. In this case, the electrons behave the same as if there were no SO coupling. In contrast, holes in the valence band (VB) are described by p-like atomic orbitals with an orbital angular momentum $l=1$. The total angular momentum for holes in the valence band is thus $j=3 / 2$ and $j=1 / 2$. The respective band pictures with and without SOC are shown in Fig. 2.4.

The states with $j=1 / 2$ reside in the so-called split-off band and are separated from the states with $j=3 / 2$ by the spin-orbit gap $\Delta_{0}$. The states with $j=3 / 2$ are degenerate at the top op the valence band $(k=0)$. For non-zero $k$-vectors, these states split further into heavy holes $(\mathrm{HH})$ with $m_{j}= \pm \frac{3}{2}$, and light holes (LH) with $m_{j}= \pm \frac{1}{2}$ - so-named for their respective effective mass.

\section{The valence band in quantum wells}

The behaviour of the valence band in quantum wells is dictated by the confinement of the wavefunction. Size-quantization lifts the degeneracy of the $j=3 / 2$ states. The resulting difference in energy of the $\mathrm{HH}$ and $\mathrm{LH}$ states at $k=0$ is called HH-LH splitting. ${ }^{40}$ Anisotropic dispersion for holes means that the dispersion in the direction of motion is such that for in-plane motion $\left(k_{\|}\right)$, the character of the "heavy-holes" and the "light-holes" is reversed, and the dispersion of HHs is now steeper than for LHs. With HH-LH splitting, the states mix and there is an avoided crossing. This behaviour is sketched in Fig. 2.4b for both the dispersion in $k_{z}$ and $k_{\|}$.

However, even if $k_{\|} \neq 0$ it is expected that the confinement wavevector $k_{z}$ is much larger than the Fermi wave vector $k_{F}, k_{z} \gg k_{F}$, so that the pure $\mathrm{HH}$ and LH states are the ground-states.

\section{g-tensor anisotropy in 2D quantum wells}

The spin degree of freedom due to SO coupling is also affected. In quasi-2D systems, it is predicted that for the high-symmetry Si [001] direction, states with 
$k_{\|}=0$ are expected to be pure HH and LH states. Furthermore, the orientation of $\mathrm{HHs}$ in $2 \mathrm{D}$ systems is expected to be parallel to the confinement axis. ${ }^{\text {BW }}$

The Zeeman term in the hamiltonian reads: ${ }^{[31}$

$$
H_{Z}=-2 \kappa \mu_{B}\left(\begin{array}{cccc}
\frac{3}{2} B_{z} & \frac{\sqrt{3}}{2} B_{-} & 0 & 0 \\
\frac{\sqrt{3}}{2} B_{+} & \frac{1}{2} B_{z} & B_{-} & 0 \\
0 & B_{+} & -\frac{1}{2} B_{z} & \frac{\sqrt{3}}{2} B_{-} \\
0 & 0 & \frac{\sqrt{3}}{2} B_{+} & -\frac{3}{2} B_{z}
\end{array}\right)
$$

where $B_{ \pm}=B_{x} \pm i B_{y}$ and $\kappa$ is the isotropic g-factor, and the basis is defined for the $\mathrm{HH}$ eigenfunctions in the order $m=3 / 2,1 / 2,-1 / 2,-3 / 2$.

For an out-of-plane B-field the off-diagonal matrix elements are zero, and it can be seen that there is a Zeeman splitting $\Delta_{H H}=6 \mu_{B} \kappa B_{z}$ for the HH states, and $\Delta E_{L H}=2 \mu_{B} \kappa B_{z}$. There is no direct coupling between the HH states proportional to $\kappa$, and therefore the coupling of the $\mathrm{HH}$ states to the magnetic field is expected to be suppressed.

\subsection{Defects}

A large body of work exists on the physics of (point) defects, we will introduce here some of the concepts associated with defect physics. For a more in-depth exploration we refer to Alkauskas et al. [41], Freysoldt et al. [42], and Estreicher [4:3].

Electrically active defects are often characterized by a charge state, and a certain structural configuration. An important parameter is the parameter $U$, sometimes also called the correlation energy. For a defect with three charge states, positive $\mathrm{D}^{+}$, neutral $\mathrm{D}^{0}$ and negative $\mathrm{D}^{-}, U$ is defined as the energy difference when taking $2 \mathrm{D}^{0}$ states and moving one electron from one defect to the other, thus producing $\mathrm{D}^{+}$and $\mathrm{D}^{-}$. This energy is reduced by any structural reconfiguration. This reaction is characterized as follows. 14

$$
2 \mathrm{D}^{0} \longrightarrow \mathrm{D}^{+}+\mathrm{D}^{-}+|U|
$$

When $U$ is positive, it follows that the stable configuration is of $2 \mathrm{D}^{0}$. This also means that the defect is paramagnetically active, since the state of the electron that resides on a defect level can be split with a magnetic field and driven in electron-spin resonance (ESR). If $U$ is negative however, there is a net energy gain associated with configuration $\mathrm{D}^{+}, \mathrm{D}^{-}$. Therefore $2 \mathrm{D}^{0}$ is not thermodynamically stable. 45

The transition level $\mu(+, 0)$ is defined as the value of the electrochemical potential $\mu$ for which the energies of the two charge states, the positively and neutral charged defects, are equal. When $\mu<\mu(+, 0)$ the defect is postively charged in the ground 
state. When $\mu>\mu(+, 0)$, it is neutral in the ground state. Considering the bandpicture of where these levels reside, the Fermi level $\left(\mu_{F}=E_{F}\right)$ determines the filling of the charge-states. This can be seen in Fig. 2.5b. In experiment, these levels are broadened and described by the the density of interface states $D_{i t}$, akin to the density of states (DOS) as used in solid-state band picture descriptions, and is given in units of $\mathrm{cm}^{-2} \mathrm{eV}^{-1}$.

If the positive and negative charge states are close to the valence-band maximum (VBM) and conduction-band minimum (CBM), they can be interpreted as a donorlike and an acceptor-like state, respectively. This is why these types of charge defects are called amphoteric. This can be seen in Figure 2.56 where the Fermi level $E_{F}$ results in the donor-like states begin half-filled, and the system here is thus slightly positive.

\subsection{1 $P_{b}$ centers}

$\mathrm{P}_{\mathrm{b}}$ centers are paramagnetic charge defects that form at the interface between $\mathrm{Si}$ and $\mathrm{SiO}_{2}$. Originally discovered by Nishi et.a ${ }^{[35}$ in a family of paramagnetic - hence the "P" - defects $\mathrm{P}_{\mathrm{a}}, \mathrm{P}_{\mathrm{b}}$, and $\mathrm{P}_{\mathrm{c}}$. The $\mathrm{P}_{\mathrm{b}}$ center is the only electrically active defect and therefore the most relevant in technological applications thus far. We discuss a structural picture of this defect in Chapter 3 .

At the $\mathrm{Si}$ (100) interface, two types of defect can be further distinguished: the $\mathrm{P}_{\mathrm{b} 0}$ and $\mathrm{P}_{\mathrm{b} 1}$ center. Bulk properties of $\mathrm{P}_{\mathrm{b}}$ centers have been measured using diverse techniques such as electron-spin resonance (ESR), ${ }^{46,47}$ capacitance measurements versus frequency $(\mathrm{CV})$, and deep-level transient spectroscopy (DLTS). The picture that emerges from these works is that the $\mathrm{P}_{\mathrm{b}}$ center is an amphoteric defect that has 3 charge-state configurations: the positive $\mathrm{D}^{+}$, neutral $\mathrm{D}^{0}$, and negative $\mathrm{D}^{-}$ with 0,1 , or 2 electrons on the $\mathrm{P}_{\mathrm{b}}$ center, respectively ${ }^{48}$ (Fig. ए.5 $)$. The transition levels of these states $(+, 0),(0,-)$ are located at $0.25 \mathrm{eV}$ and $0.85 \mathrm{eV}$ above the valence band maximum, respectively. The $D_{i t}$ as determined in CV measurements shows the three charge states with the transition levels being significantly broadened. This is shown for different annealing biases in Fig. ए.

The second type of $\mathrm{P}_{\mathrm{b}}$ center, the $\mathrm{P}_{\mathrm{b} 1}$ center, is generally not considered to be electrically active and has two levels located in the bandgap distributed slightly below the midgap. ${ }^{49}$

The orbital configurations of the $\mathrm{P}_{\mathrm{b}}$ center change with charge state where the positively charged $\mathrm{D}^{+}$exhibits $\mathrm{sp}^{2}$-hybridization with an empty p-orbital, the neutral $\mathrm{D}^{0}$ exhibits $\mathrm{sp}^{3}$-hybridization with one singly occupied $\mathrm{sp}^{3}$ hybrid orbital, and the negatively charged defect exhibits $\mathrm{s}^{2} \mathrm{p}^{3}$-hybridization with a doubly occupied lone pair. ${ }^{44}$ 
(a)

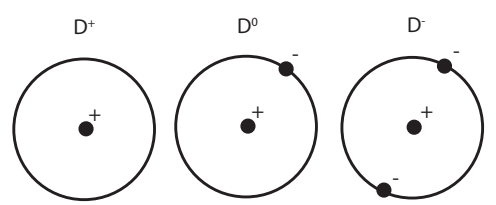

(b)

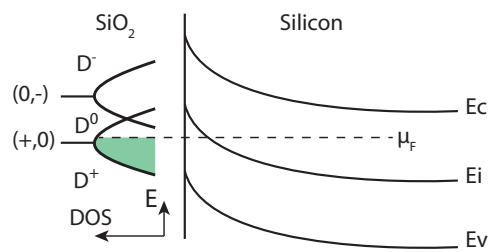

Figure 2.5: $\mathrm{P}_{\mathrm{b}}$ center defect levels. (a) The three charge states: $\mathrm{D}^{+}, \mathrm{D}^{0}$, and $\mathrm{D}^{-}$ of the $\mathrm{P}_{\mathrm{b}}$ center. (b) $\mathrm{P}_{\mathrm{b}}$ center level filling as a function of Fermi level at the $\mathrm{Si} / \mathrm{SiO}_{2}$ interface in weak inversion. Here $E_{i}$ is the intrinsic Fermi level, and $\mu_{F}$ the equilibriated Fermi level. After Entner [50]

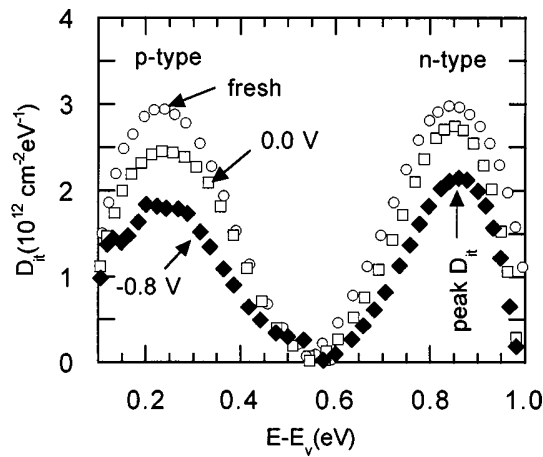

Figure 2.6: Density of interface states $D_{i t}$ for $\mathrm{P}_{\mathrm{b}}$ centers measured with the CV method. The density is shown as a function of bias during annealing. From Ragnarsson and Lundgren [5]].

\section{Theoretical calculations}

Theoretical calculations of the energy levels of $\mathrm{P}_{\mathrm{b}}$ centers have shown that the transition levels $(+, 0),(0,-)$ are located at 0.20 and $0.80 \mathrm{eV}$ above VBM consistent with experimental values 0.26 and $0.84 \mathrm{eV} . \underline{52}$ In order to correctly reproduce the band gap, theoretical calculations often rely on a hybrid approach where an adhoc mixing of exact hartree-fock exchange energy into an other local spin-density approximation. This tuning parameter controlling mixing is thus based on experimental observation. A review of these so-called hybrid density functionals used on defect levels can be found in Alkauskas et al. [53].

A remaining problem of these methods in determining the position of levels of point defects in the band gap is the "band-edge problem". While the experimental band gap can be properly reproduced in these methods, the band edge has been shown to vary quite substantially using different methods. It is unclear how this would effect calculations when there is a slanted potential, such as in a quantum 
well. Due to this shifting of band edges with respect to an average electrostatic potential, an accuracy greater than $0.1 \mathrm{eV}$ is not expected. ${ }^{12}$

Theoretical DFT calculations of isolated dangling bonds on silicon have shown defect transition levels located at $11 \mathrm{meV}$ above the VBM. ${ }^{[4}$ In contrast to ensemble calculations that take into consideration a repeating unit cell, this is much closer to the VBM.

\subsubsection{Passivation of $\mathrm{P}_{\mathrm{b}}$ centers}

The passivation of $\mathrm{P}_{\mathrm{b}}$ centers is typically achieved by introducing hydrogen to the dangling bond at sufficiently high energies, such that the dangling bonds covalently bond to a hydrogen atom. This shifts the transition levels $(+, 0)$ and $(0,-)$ out of the bandgap and decreases $D_{i t} \cdot{ }^{55,56}$ The activation energies for this passivation are $E_{a}=1.5 \mathrm{eV}$ for the $\mathrm{P}_{\mathrm{b} 0}$ defect at the $\mathrm{Si}(100)$ interface,, 57 and $E_{a}=1.66 \mathrm{eV}$ for the $\mathrm{P}_{\mathrm{b}}$ defect at the $\mathrm{Si}(111)$ interface. ${ }^{58}$ The surface potential however is of influence on the activation energies required for passivation, and have been shown to vary with the position of the Fermi level between $E_{a}=1.5 \mathrm{eV}$ and $E_{a}=1.8 \mathrm{eV}$.

\subsubsection{Role of $\mathrm{P}_{\mathrm{b}}$ centers in MOSFETs}

The simplest way to picture the influence of $\mathrm{P}_{\mathrm{b}}$ centers in MOSFETs is to consider that $\mathrm{P}_{\mathrm{b}}$ centers are amphoteric in nature, i.e. they can be charged both positively and negatively. $\mathrm{P}_{\mathrm{b}}$ centers therefore can act as an additional capacitor in series with the oxide that separates metal and semiconductor. Before reaching the surface potential $\phi_{F}$ required for charge accumulation in the MOSFETs, this additional capacitor has to be charged, thus delaying the onset of conductivity. It is therefore seen that the threshold voltage $V_{t h}$ (for both p-type and n-type) is directly related to the spatial density of $\mathrm{P}_{\mathrm{b}}$ centers. 


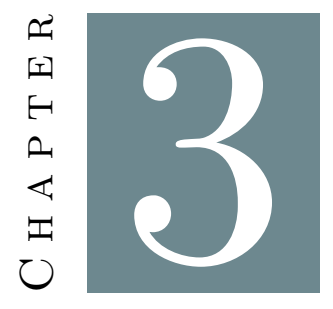

\section{Quantum dot heterostructures}

Dealing with the fragility of the quantum state is one of the key issues on the road to meet the limits posited by quantum computation schemes. "II It is the entanglement of quantum states to states in an unknown environment which is the driver for decoherence. The properties of the environment therefore dictate the performance of a qubit in a proposed quantum computer. Creating these qubits in the solid state means that the environment consists of the many different materials and structures used in device construction. Quantum dots have been created by confining carriers in $\mathrm{AlGaAs} / \mathrm{GaAs}^{5.9}$ or $\mathrm{Si} / \mathrm{SiGe}$ heterostructures, ${ }^{601}$ to dopant atoms in a host material, in SiGe nanohuts, ${ }^{611} \mathrm{Si}$ and $\mathrm{Si} / \mathrm{Ge}$ nanowires, etched mesas on silicon-on-insulator, ${ }^{6.3}$ and planar MOS structures. ${ }^{64}$ The intrinsic properties of these (material) systems give rise to interactions detrimental to qubit creation and readout.

The hyperfine interaction of the host material and the qubit is one such effect. The non-zero-spin isotopes in a material create a nuclear-spin-bath and cause decoherence of the quantum state. This is the basis for the proposal for the use of isotopically purified silicon as a host material for quantum dots. ${ }^{65,66}$ The purified silicon, now containing predominantly ${ }^{28} \mathrm{Si}$, results in a zero-nuclear-spin isotope system, and eliminates the Overhauser field.

Field noise, such as charge- and spin-noise ${ }^{3.3}$ can also influence the lifetime of the quantum state. One strategy to deal with these fluctuations is to tune the quantum dots to certain regimes in phase space where the energy levels of interest are insensitive to these fields. This can happen in the clock transition of Bi dopants in $\mathrm{Si}, \stackrel{67}{6}$ by dressing qubits and tuning them appropriately, $\stackrel{\text { [8] }}{,}$ or for hybrid quantum dots. ${ }^{69}$ Another effect that can influence quantum states are fluctuations in the 
electrochemical potential at longer timescales. These have been shown to occur due to charge offsets fluctuating over time in glassy media and their intrinsic twolevel systems (TLS). $\underline{34, \pi 0}$

Finally, unintentional quantum dots, charge traps, or charge defects can influence a desired quantum state. This class of effects can manifest when the scale of the wavefunction of the charge carriers involved is equal to the scale of (unintended) features or variations in the structure. In silicon, the electrons have a larger transverse effective mass $\left(m_{t}^{*}=0.19 m_{0}\right)$ as compared to e.g. the electrons in GaAs $\left(m^{*}=0.067 m_{0}\right)$. The electrons in silicon therefore have a smaller wavepacket. Smaller features in the structure, or atomic scale defects can thus play a more significant role.

The definition and the quality of the quantum state is not only determined by the host material in which the quantum state mostly resides. It is the entire heterostructure which, through various effects, interacts with the quantum state. Therefore, great care must be taken in the creation of the heterostructure and materials, in order to realize an ideal quantum dot.

Before we continue, let us first define more concisely what we mean when we talk about a heterostructure: A structured combination of different materials intended to effect a phenomenon. The phenomenon here is a quantum dot. Next, we can use this definition to identify what criteria we can identify as crucial to effect the phenomenon. In this case we can already claim that we want charge stability, long spin-lifetimes, and no unintentional quantum dots. Based on these aspects we can look at the heterostructure and its fabrication and tease out what parameters have to be satisfied. The definition of a heterostructure put forth here will by no means be complete but is more intended to serve us as a framework by which we will look at the problem.

This chapter will first introduce the planar heterostructure, as a means of effecting a quantum dots electrostatically in a semiconductor. We will then introduce the three heterostructures used in this work, based on the planar heterostructure. Then we will go through the layer growth techniques relevant to this work in particular and to heterostructure creation in general. Next, we will review the Si planar quantum dot heterostructure in its entirety and identify key points in the heterostructure where effects might occur which are deleterious to quantum dot quality. Finally, we will take an in depth look at the role of annealing and hydrogen in our heterostructures.

\subsection{The electrostatically defined quantum dot heterostruc- ture}

The general working principle of the planar quantum dot heterostructure closely resembles that of a MOSFET - in that the band structure of the semiconductor is manipulated electrostatically by local gate electrodes, which are separated 
electrically by an insulating layer from the silicon underneath. The electrodes control the flow of carriers in the device and shape the potential profile required for quantum dot formation. The lateral configuration of the electrodes determines the region where the potential can be manipulated, while the voltage applied to individual gate electrodes creates the electric field that can control the height of the potential. When the potential applied to the gate electrodes is sufficient, the conduction band (CB) or valence band (VB) is pulled above or below the Fermi level so that states become available for transport, confining the carriers to a $2 \mathrm{D}$ carrier gas 1-2 nanometers thick.

In order to measure conductance through the device, source and drain regions are created by doping and ohmic contacting. Implantation regions form source and drain reservoirs and can be ohmically contacted to measure conductance through the device.

Figure 3 shows the ideal case of the heterostructure with the resulting potential profile being two tunnel barriers and a quantum dot in the middle, for the applied potential on the gate electrodes.

\subsubsection{Fabrication strategy}

All devices in this work are made using the same fabrication strategy.

First, the "big" structures, down to approximately 2 micron resolution, are defined at wafer scale using photolithography. This entails the oxidation of Si to form $\mathrm{SiO}_{2}$, the formation of the implantation regions for the source and drain contacts, creation of the (high quality) oxide, and the metallization of the electrodes which can be contacted later for measurement. Figure 5.5 shows the top view of the structures created in this process.

Then, the smaller structures can be defined. Electron-beam lithography (EBL), followed by evaporation and liftoff is used to define the sub-micron electrode gates. The device usually consists of more than one layer of electrodes, and therefore an insulating layer, electrically separating the electrode layers electrically, is needed.

Separating the fabrication process in two steps at different scale allows for rapid iteration of device configurations, which are otherwise limited by the speed at which the definition of gate electrodes with EBL takes place.

\subsubsection{The ambipolar concept}

The behaviour of charge carriers in the valence and conduction-band is different, and both charge carriers have different properties. As an example, in bulk and at band-minimum, the longitudinal and transverse effective mass for transport for electrons is reported as $m_{e, l}^{*}=0.98 m_{0}, m_{e, t}^{*}=0.19 m_{0}$, while for holes the effective mass is $m_{h h}^{*}=0.49 m_{0}, m_{l h}=0.16 m_{0}, \frac{}{10}$ with $m_{0}$ the rest mass of the electron. Furthermore, the spin-orbit coupling for holes in Si is higher than for their coun- 


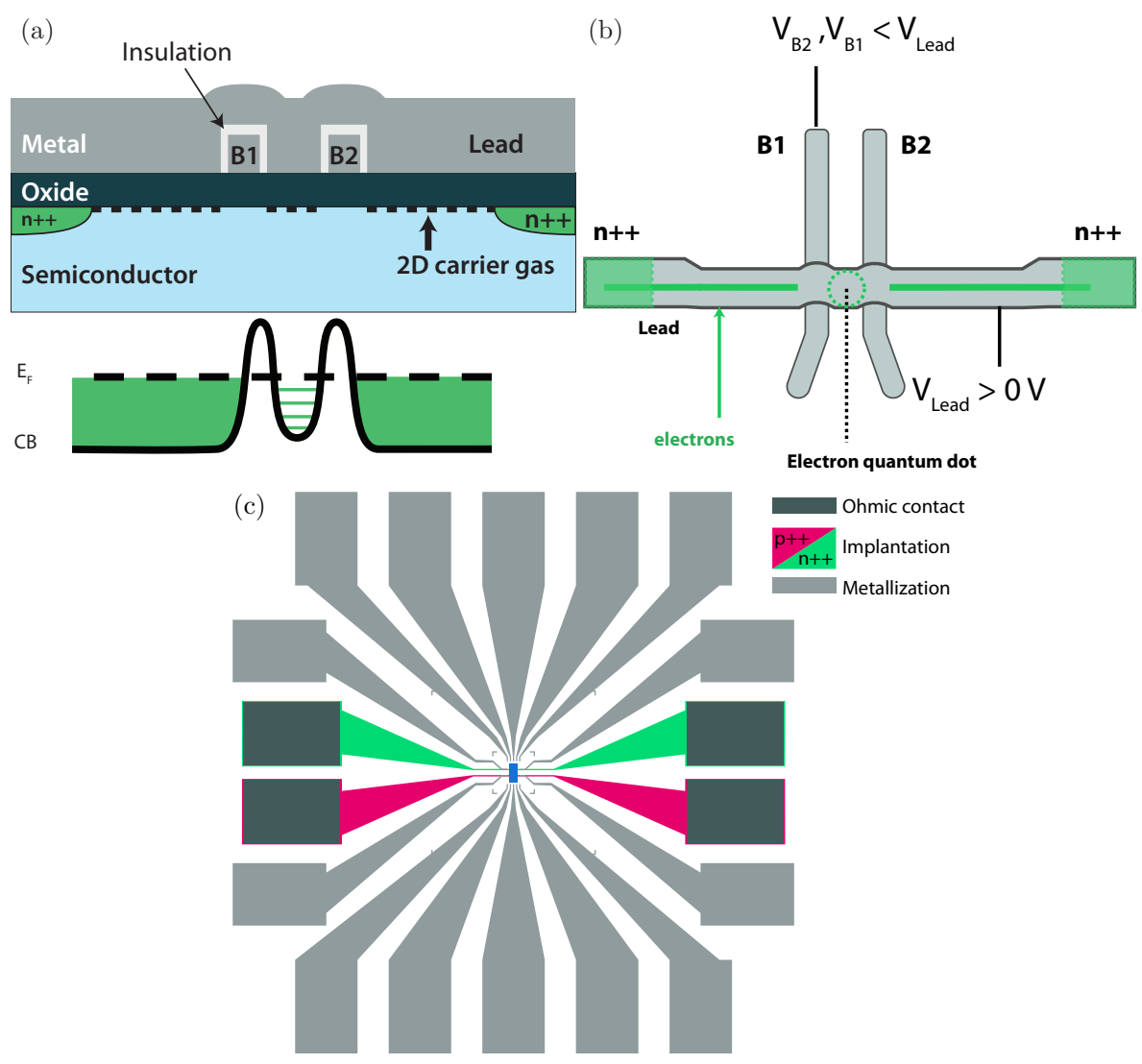

Figure 3.1: Planar quantum dot heterostructure. (a) a cross-sectional view for an n-type implantation with the desired potential profile of a quantum dot for electrons. (b) a top-view. (c) Chip design at mesoscale with a design area of $2 \times 2 \mathrm{~mm}^{2}$, showing the implantation regions in red and green for holes and electrons respectively, ohmic contacts in dark blue and the connecting gate architecture in light grey. The center of the device area is comprised of high-quality oxide (approximately $70 \times 30 \mu \mathrm{m}^{2}$ ). 
terparts in the conduction band, since the orbital angular momentum is non-zero. The criteria for quantum dot formation therefore also differ.

To explore this, ambipolar devices can be created where both holes and electrons can be used as charge carriers. modified to create create $\mathrm{p}$ - and n-type implantation regions at either end of the device. Which carrier is used to transport charge is controlled by gating the intrinsic silicon (Figure [.2. $)$. A negative potential on the gate will pull the VB above the Fermi level, making hole states available for transport. Conversely, applying a positive potential on the gate will push the CB below the Fermi level and make electron states available for transport. We call these modes of operation the hole and electron operation regime (Figure 3.2b). The ability to transport either electrons or holes in the same crystalline environment allows for the probing of states close to either the $\mathrm{CB}$ or $\mathrm{VB}$ of the semiconductor, while discounting fabricational variations that would otherwise exist when measuring these effects from one device to the next.
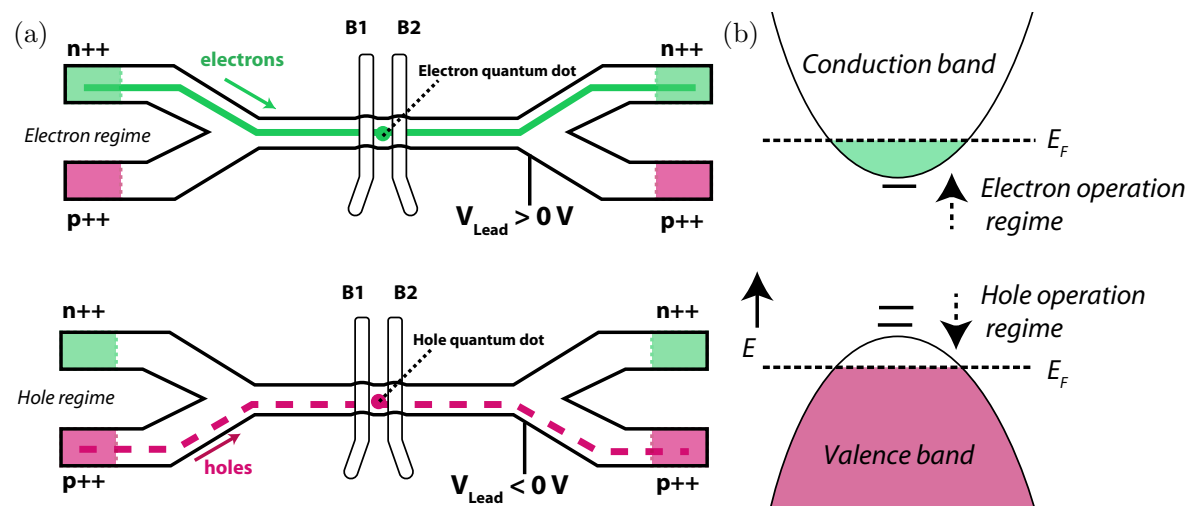

Figure 3.2: The ambipolar device concept. (a) the two modes of operation of the ambipolar device. By applying a negative voltage holes can flow from the source and drain implantation region. A positive voltage allows electrons to be used as charge carriers. (b) Schematic overview of the bandgap of silicon, including possible states in the bandgap. Not shown here is the indirect nature of the bandgap. The range of operation of the ambipolar device is apparent, allowing probing of states close to the valence band or conduction band, depending on the operation mode of the device. 


\subsubsection{Variations on a theme: the three heterostructures used in this work}

In this work, we will encounter three variations on the basic heterostructure discussed in Section $[$.$] . The different designs attempt to address several issues,$ which we will discuss later in this chapter.

All heterostructures use $\mathrm{SiO}_{2}$ as the first insulating layer. The first heterostructure (H1) uses $\mathrm{Al}$ as the material for gate electrodes, based on the recipe introduced by Angus et. al..$^{[01}$ and can be seen in Figure $3.3(\mathrm{H} 1)$. Aluminum has the advantage that an insulating layer is easily created between the electrodes by thermal oxidation. In the second heterostructure $(\mathrm{H} 2)$, a $5 \mathrm{~nm}$ layer of $\mathrm{Al}_{2} \mathrm{O}_{3}$ grown at $250{ }^{\circ} \mathrm{C}$ is added between the $\mathrm{Al}$ and the $\mathrm{SiO}_{2}$, and a capping layer of $\mathrm{Al}_{2} \mathrm{O}_{3}$ (not shown) is finally grown at $100^{\circ} \mathrm{C}$. The third heterostructure (H3) substitutes the $\mathrm{Al}$ for $\mathrm{Pd}$ as the gate electrode material, and maintains the inter- and capping-layer of $\mathrm{Al}_{2} \mathrm{O}_{3}$.

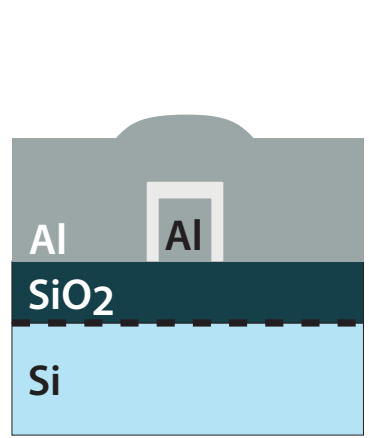

(H1)

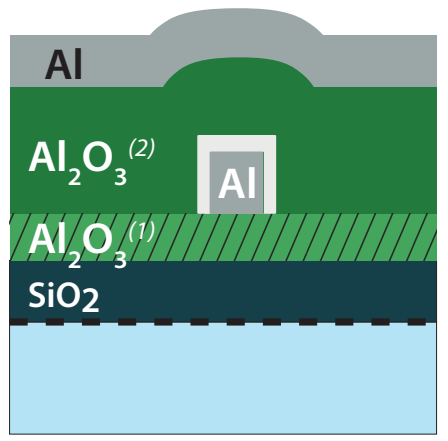

(H2)

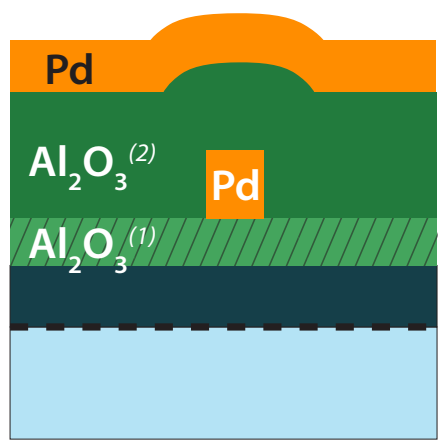

(H3)

Figure 3.3: Three heterostructures with (H1) the Al-gate standard architecture (H2) the architecture including a additional, separately grown (1),(2), $\mathrm{Al}_{2} \mathrm{O}_{3}$ layers and (H3) the architecture using Palladium as a gate electrode material. The source and drain implanted regions have been omitted here.

\subsection{Layer synthesis techniques}

For a well-defined heterostructure, it is essential that the properties of the materials constituting a heterostructure are controlled. Here we will shortly discuss the working principles of these techniques and discuss some of their finer points, which we will relate in Section [3.3 to how they might influence QD formation. 


\subsubsection{Physical vapour deposition}

\section{Thermal evaporation}

Thermal evaporation utilizes resistive heating of a crucible (typically made from $\mathrm{W}$ or Mo) filled with the material to be evaporated. The material is heated until it begins to sublimate. For deposition pressures of $10 \times 10^{-6}<p_{\text {dep }}<10 \times 10^{-2}$ Torr the evaporant can be considered to be in the ballistic regime. ${ }^{\square \pi}$ The evaporant travels through the evacuated chamber and subsequently lands on the substrate, initiating the growth of a thin film.

For evaporation of metals, there are a few parameters of note. First, the rate of evaporation, controlled primarily by temperature, determines the grain size of the thin film. The difference in temperature between the evaporated material and the substrate determines the grain size of the polycrystalline film. This is called the homologous temperature $T_{h}$. The second aspect is the purity of the evaporated material. Since the evaporated material is situated in a thermally heated crucible, there is the possibility of contamination from the crucible material.

(a)

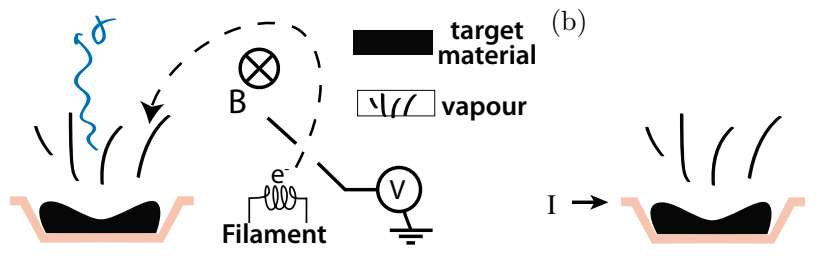

Figure 3.4: Evaporation techniques. (a) E-beam evaporation, with the target metal being heated by energized electrons. The $\gamma$ indicates the $\mathrm{X}$-ray radiation that is emitted in this process. (b) Thermal evaporation. The crucible is resistively heated by current $I$, leading to heating and subsequent evaporation of the target material.

\section{Electron-beam evaporation}

In this evaporation technique, electrons are excited out of a filament by resistive heating and subsequently accelerated by an electric field to a high voltage (typically $\approx 10 \mathrm{keV}$ ). The electron trajectory is curved by a magnetic field and the electrons directed to the target material. The energetic electrons hit the target material and heat it. The subsequent vapour travels through the vacuum chamber and is deposited on the substrate.

One of the advantages of e-beam evaporation, in contrast to thermal evaporation, is that there is no real danger of contamination by the crucible. Because the e-beam heats the sample locally, a small puddle is formed inside the target material. This puddle is thus only in contact with the same constituent material. 
Putting e-beam evaporation at a disadvantage however, is that X-rays are generated in the impact of the high-energy electrons with the target material. Possible damage to the substrate is therefore a concern.

Grain size in this system is also controlled by the homologous temperature. Exploiting this, it has been shown for Al that cooling of the substrate can greatly improve the grain size of the thin film. ${ }^{[8]}$ For typical evaporation rates of around 1-5 $\AA$ /s grain-sizes for Pd have been reported to $15-40 \mathrm{~nm},{ }^{[\mathrm{TM}}$ while for $\mathrm{Al}$ the grain sizes vary from $20-40 \mathrm{~nm}$ for the same range of rates.

\subsubsection{Atomic Layer Deposition}

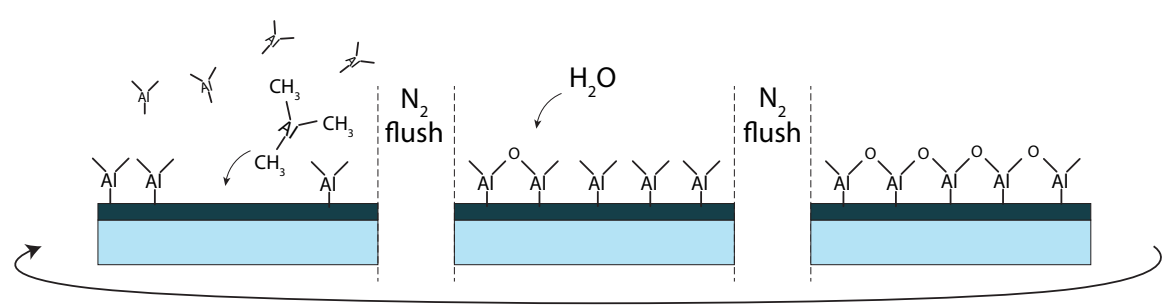

Figure 3.5: Atomic layer deposition process showing precursor gases TMAH, flush with $\mathrm{N}_{2}, \mathrm{H}_{2} \mathrm{O}$, and subsequent $\mathrm{N}_{2}$ flush.

Atomic layer deposition (ALD) is a technique independently discovered in the $60 \mathrm{~s}$ in the Soviet Union and in the 70s in Finland. ${ }^{\mathbb{1}}$ It makes possible the controlled layer by layer growth of oxides, metals, fluorides, nitrides, sulfides, carbides, etc. ALD is a self-limiting process and is a subset of chemical vapour deposition. Its main advantages are the low temperatures at which the process can be used, precise control over film thickness, and conformal coverage of the resulting film.

In general two precursor gases are used. In this work ALD is used for the growth of $\mathrm{Al}_{2} \mathrm{O}_{3}$. We will use this as an example to illustrate the process. Figure 5.5 shows the process from start to finish. In the first step Tetramethylammonium hydroxide (TMAH) is introduced to the chamber in gaseous form, together with a stream of $\mathrm{N}_{2}$. The TMAH molecules chemisorb on the surface (reaction equation $\left[\begin{array}{l}\text {. } \\ \text { ) until }\end{array}\right.$ the layer fully covers the surface and subsequent molecules are unable to attach to the chemically stable layer. The next step is to purge the chamber with $\mathrm{N}_{2}$ leaving only the adsorbed TMAH monolayer. This is the point where the second precursor is introduced - in this case $\mathrm{H}_{2} \mathrm{O}$. The $\mathrm{H}_{2} \mathrm{O}$ reacts with the TMAH, which, to first order, oxidizes it through reaction equation [2.

$$
\begin{array}{clr}
\mathrm{Al}\left(\mathrm{CH}_{3}\right)_{3}(\mathrm{~g})+\mathrm{Si}-\mathrm{O}-\mathrm{H}(\mathrm{s}) & \longrightarrow \mathrm{Si}-\mathrm{O}-\mathrm{Al}\left(\mathrm{CH}_{3}\right)_{2}(\mathrm{~s}) & +\mathrm{CH}_{4} \\
2 \mathrm{H}_{2} \mathrm{O}(\mathrm{g})+\mathrm{Si}-\mathrm{O}-\mathrm{Al}\left(\mathrm{CH}_{3}\right)_{2}(\mathrm{~s}) & \longrightarrow \mathrm{Si}-\mathrm{O}-\mathrm{Al}(\mathrm{OH})_{2}(\mathrm{~s}) & +2 \mathrm{CH}_{4}
\end{array}
$$


The reactants and precursor gas are then flushed with another $\mathrm{N}_{2}$ purge, resulting in a monolayer of $\mathrm{Al}_{2} \mathrm{O}_{3}$. After this the cycle starts anew. The self-limiting coverage of a single covering layer in each cycle leads to highly conformal layers and excellent control of film thickness.

The simplified reaction equation as sketched in equation 5.2 does not do the entire process justice, as the use of $\mathrm{H}_{2} \mathrm{O}$ as a precursor gas has been shown to create hydrogen as a reaction by-product in the ALD-cycle. ${ }^{82}$ This causes ALDgrown $\mathrm{Al}_{2} \mathrm{O}_{3}$ to have hydrogen content at 2-3 at.\%. Experiments with deuterated water as a precursor show deuterium diffusing from the $\mathrm{Al}_{2} \mathrm{O}_{3}$ film toward the $\mathrm{Si}$ interface at annealing temperatures of $400{ }^{\circ} \mathrm{C}$, where dangling bonds are passivated and lead to a lower density of interface states $D_{i t}$.

The growth temperature of the $\mathrm{Al}_{2} \mathrm{O}_{3}$ determines the density of the oxide. The hydrogen content will be shown to have an important consequence later on in Section [3.4.

\subsection{3 $\mathrm{SiO}_{2}$ growth and oxidation}

The growth of $\mathrm{SiO}_{2}$ is accomplished by simply introducing an oxidant to silicon at high temperature. The oxidant diffuses through the silicon and oxidizes the silicon from the top up to the desired depth.

Wet oxidation is generally used to create thick, but qualitatively poor, oxides at a rate of $400 \mathrm{~nm} / \mathrm{h}$ at $1000{ }^{\circ} \mathrm{C}$ and takes places by introducing $\mathrm{H}_{2} \mathrm{O}$ as the oxidant. Dry oxidation occurs in $\mathrm{O}_{2}$-containing ambient and oxidizes the silicon at rates far slower than for wet oxidation $(<100 \mathrm{~nm} / \mathrm{hr})$. The latter process yields a denser oxide with fewer defects and higher breakthrough voltages.

Oxygen, introduced at high temperature, will oxidize the top layer of the silicon first. The newly created oxide takes up more volume than was occupied by silicon before oxidation. This decrease in density allows more oxygen to penetrate deeper into the silicon, and oxidizes at the newly formed $\mathrm{Si} / \mathrm{SiO}_{2}$ interface. The process continues and the $\mathrm{Si} / \mathrm{SiO}_{2}$ oxidation front progresses further down into the substrate. Because of the increase in volume, taking place at $900{ }^{\circ} \mathrm{C}$, the silicon lattice will be uniformly stressed by the $\mathrm{SiO}_{2} \cdot{ }^{8.3}$

The Deal-Grove model describes the oxidation reaction as occurring at the $\mathrm{Si} / \mathrm{SiO}_{2}$ oxidation front. It describes the diffusion of the oxidant through the already formed oxide in terms of concentration differences, for which the model assumes steady-state conditions. Discrepancies of the Deal-Grove model for thin oxides have lead to new insights and it has been shown that a more complete model involving Si emission from the surface describes thin oxides better. ${ }^{84,85}$ The quality of the oxide can be further improved by the addition of chlorine-containing gas during oxidation. The reduction of atomic silicon emission from the silicon by the chlorine is most likely responsible for this.

It is important to note that the oxidation process is not isotropic. Should thermal oxidation start at a surface that is made atomically flat; that flatness would 
not propagate to the $\mathrm{Si} / \mathrm{SiO}_{2}$ interface under normal conditions. Furthermore the conditions for oxidation leads to a wide array of interface morphologies. $\stackrel{87-89}{ }$ One possible solution for this might be a new technique using a microwave excited high density plasma with low electron temperature. 90,90 The free radicals created in this process have been shown to lead to a more abrupt $\mathrm{Si} / \mathrm{SiO}_{2}$ interface and to preserve the atomically flat nature of as-prepared wafers. ${ }^{42}$ The nature of this interface has not been fully explored yet, save for theoretical studies that suggest a phase of atomically flat, and stable $\mathrm{SiO}_{2}$ can exist. ${ }^{.33}$

\subsection{Defects in quantum dot heterostructures}

As mentioned previously, the fragile nature of quantum states means there are many effects that do not affect mesoscopic devices, yet play a big role in nanodevices.

We identify roughly three categories for heterostructures. These categories will serve us as rough guides to determine where effects occur in heterostructures, which in turn might influence design considerations. The first category consists of the inherent material properties of the constituents of the heterostructure. These are mainly determined by the initial growth process, but can also be influenced during processing. The second category concerns interfaces, which can manifest a-priori unexpected phenomena. This is a broad and active area of research unto itself which has garnered interest due to its fundamental physical concepts involving e.g. the breaking of symmetries.

The third are morphological effects. We can think e.g. of the morphology of the gate layout intended to form intentional quantum dots. However, there are many more unforeseen morphological effects which can occur and must be considered.

We will looks at these categories, guided by the three main heterostructures used in this work, as introduced in Section B...3.

\subsubsection{Materials}

We will start by examining the basic building blocks of heterostructures, their materials. It is the intrinsic material properties of e.g. silicon that make it a highly valued candidate for quantum computing. Let us briefly review what material properties influence the formation of our quantum dots.

\section{High-quality oxide}

The creation of an inversion layer in silicon requires the application of a voltage. Voltages are applied to electrodes typically separated from the silicon by only a few tens of nm of oxide. The electric fields over this short distance are thus large, and on the order of $\mathrm{MV} / \mathrm{cm}$. The oxide should be able to withstand these 
high voltages and avoid pinhole defects that occur when reaching the so-called breakthrough voltage.

For all three heterostructures in this work, the process for creating a high quality oxide is the same. The addition of chlorine during growth improves our breakthrough voltage of the oxide. Without it, significantly smaller breakthrough voltages were observed after dopant activation in a rapid thermal annealing process (RTA).

\section{Damage incurred by processing}

However perfect the material grown during a process step, it is possible that high energy processes during further processing of the heterostructure causes damage. The thermal budget is a common indicator used to signify the maximum allowed thermal load before a device will be too damaged. Process damage manifesting as an increase in defect density is also known to occur when using electron-beam evaporation. ${ }^{\sqrt{4}}$ This is most likely caused by the X-rays that are generated during the evaporation process. Another defect that is known to be generated by high energy radiation is the $\mathrm{E}^{\prime}$ defect in $\mathrm{SiO}_{2}$. $\frac{\text {.5. }}{}$

Thus far, little research has been done on the damage electron beam lithography can cause in heterostructures. It is however reasonable to expect that the high energy electrons (as high as $100 \mathrm{keV}$ ) do interact with the materials in the heterostructure. It is not a-priori clear if a high-energy beam or a low-energy beam would be preferable, since scattering mechanisms in the entire heterostructure determine where the majority of energy of the beam is absorbed. Several models for this type of problem, based on energy deposition in (amorphous) solids have been suggested. ${ }^{\text {96, } 97}$ Low-energy patterning (up to a few $\mathrm{keV}$ ) on thin layers of resist could alleviate this problem.

All heterostructures in this work were exposed to EBL processes at energies of typically $20 \mathrm{keV}$ to $28 \mathrm{keV}$.

\section{Dewetting}

Dewetting is the breaking of continuity of an initially continuous film caused by differences in free surface energy at liquid-liquid or solid-liquid interfaces. In our case the interface consists of either $\mathrm{Al} / \mathrm{SiO}_{2}$ or $\mathrm{Pd} / \mathrm{Al}_{2} \mathrm{O}_{3}$. At elevated temperatures thin films of metal, while still below their melting point, can become mobile. Dewetting becomes energetically favourable under the right circumstances: temperature, granularity of the film, free surface energy at the $\mathrm{Al} / \mathrm{SiO}_{2}$ and vacuum/Al interface. The process can be seen in Figure [5.6]

Figure 3.7 a shows dewetting behaviour as observed in one of our samples after annealing, without the presence of an $\mathrm{Al}_{2} \mathrm{O}_{3}$ overlayer. The left $\mathrm{Al}$ electrode, labeled (1), shows signs of the start of dewetting but still has its as-deposited height of $36 \mathrm{~nm}$. The right electrode shows clear signs of dewetting. At (2) 


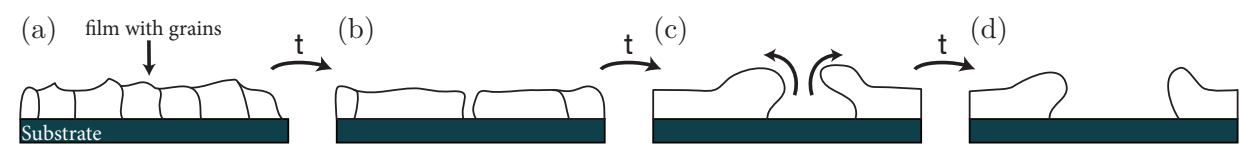

Figure 3.6: Schematic cross-sectional overview of the dewetting progress. (a) The start of the dewetting process, with the grains and grain-boundaries in the film depicted as-deposited. In the next time-step, the grains have agglomerated and formed bigger domains, with the first gaps forming due to surface tension (b). (c) A mass flux occurs as mass is transported as indicated by the arrows, and the edge of the material retracts, widening the gap between the wetted regions. (d) The process continues until an equilibrium is reached.
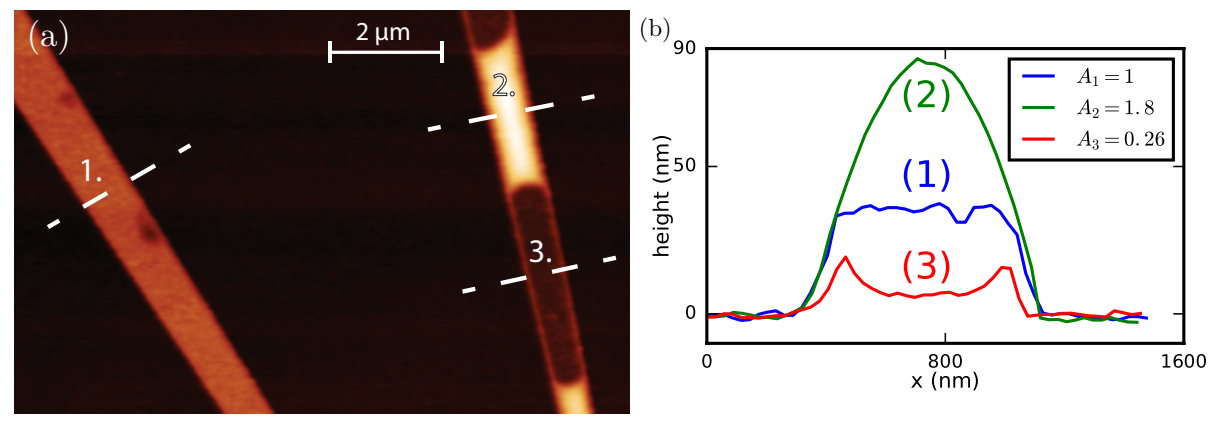

Figure 3.7: Dewetting behaviour of pure aluminum on $\mathrm{SiO}_{2}$. (a) AFM image overview of a device after annealing at $400^{\circ} \mathrm{C}$. (b) Height at the various linecuts in (a) and the areas underneath each curve normalized to $A_{1}$, the area under curve $(1)$.

the height has increased from $36 \mathrm{~nm}$ as-deposited to a maximum of roughly 90 nm. The material for this height increase has been moved from (3) to (2). The areas $A_{i}$ underneath curves taken at (1), (2), and (3) are normalized to $A_{1}$. We see that $A_{2}+A_{3} \simeq 2 A_{1}$, i.e the material "missing" from (3) has moved to (2), and thus the sum equals twice the original. At the interface region between (2) and (3) the convex/concave shape is reminiscent of the classical meniscus in e.g. water, indicating energy minimisation caused by surface tension. $\frac{98}{7}$ This can also be observed on both sides of the electrode at (3).

\section{Properties of $\mathrm{Al}$ at elevated temperatures}

At elevated temperature other effects concerning $\mathrm{Al}$ can arise. It has been reported that $\mathrm{AlO}_{x}$ hillocks are created when exposing $\mathrm{Al}$ on $\mathrm{Al}_{2} \mathrm{O}_{3}$ to a high temperature step of $600{ }^{\circ} \mathrm{C}$. 1900 The spiking of $\mathrm{Al}$ through $1.5 \mathrm{~nm}$ of $\mathrm{SiO}_{2}$ has also been reported for annealing temperature of $300^{\circ} \mathrm{C}$. stress release of $\mathrm{Al}$ at temperatures of $300^{\circ} \mathrm{C}$ to $500^{\circ} \mathrm{C}$ has been reported. ${ }^{102}$ 


\subsubsection{Interfaces}

The story of the heterostructure does not end at materials. To fully characterize the heterostructure, the interfaces between the constituent materials have to be considered. The physics of interfaces is a broad field with many interesting topics. There are many examples where the discontinuity at the interface between two materials can lead to interesting physics, such as Rashba-type spin-orbit coupling, $\frac{}{103}$ or a finite conductivity at the interface between the two insulators $\mathrm{LaAl}_{2} \mathrm{O}_{3} / \mathrm{SrTiO}_{3}$

Back to the planar quantum dot heterostructure. The three heterostructures studied have many interfaces, starting from the $\mathrm{Si} / \mathrm{SiO} 2$ to the metal/oxide interface. Naturally, the addition of an extra layer of material also introduces an additional interface where effects might occur.

\section{The $\mathrm{Si} / \mathrm{SiO}_{2}$ interface}

The first interface we will consider is the $\mathrm{Si} / \mathrm{SiO}_{2}$ interface, where a transition from crystalline $\mathrm{Si}$ to an amorphous matrix of $\mathrm{SiO}_{2}$ breaks continuity and translational symmetry. At an atomic scale, the crystalline $\mathrm{Si}$ and amorphous $\mathrm{SiO}_{2}$ are incommensurable, save for some theorized phases of $\mathrm{SiO}_{2}$ that would give an abrupt interface.

The $\mathrm{Si} / \mathrm{SiO}_{2}$ interface has been extensively studied in the context of MOSFET technology. The focus here has primarily been on defects. One of the most studied defects at this interface is the paramagnetic $\mathrm{P}_{\mathrm{b}}$ center to surface charges, decreased mobility, and the negative-bias temperature instability (NBTI) effect. $\stackrel{\square 109}{=}$ For quantum computation, $\mathrm{P}_{\mathrm{b}}$ centers have been used in coherent manipulation of spin-dependent charge-carrier recombination of phosphorus donors. ${ }^{\text {III }}$ Additionally, the coherence times of these phosphorous donors has been shown to be adversely affected in proximity to $\mathrm{P}_{\mathrm{b}}$ centers. ${ }^{\mathrm{II}}$ Defects at the interface have also been associated with random telegraph signals at low temperatures and characterized by means of single-electron spin resonance $\amalg 2, \amalg 3$ and magnetic-field dependent measurements. 14

$P_{b}$ centers, $E^{\prime}$ centers $I t$ is useful to categorize traps of influence at the $\mathrm{Si} / \mathrm{SiO}_{2}$ oxide interface by distance to the interface. We can discern, in order of increasing distance, interface traps, border traps, and oxide traps (Figure [.9b).

The two most prevalent defects are $\mathrm{P}_{\mathrm{b}}$ centers, which is an interface trap, and $\mathrm{E}^{\prime}$ centers, which are typically classified as border traps or oxide traps.

The $\mathrm{P}_{\mathrm{b}}$ center is in essence a $\mathrm{Si}$ atom with a dangling bond that has formed due to the incommensurability of the crystalline $\mathrm{Si}$ and the amorphous $\mathrm{SiO}_{2}$. Two types of $\mathrm{P}_{\mathrm{b}}$ center can be further distinguished at for $\mathrm{Si}$ (100). The $\mathrm{P}_{\mathrm{b} 0}$ center is backbonded to two $\mathrm{Si}$ atoms and one oxygen atom, while the $\mathrm{P}_{\mathrm{b} 1}$ center is backbonded to three Si atoms. This atomic configuration leading to this can be 
seen schematically in Figure [.8. For the $\mathrm{P}_{\mathrm{b} 0}$ center, the density of interface states $\mathrm{D}_{\mathrm{it}}$ in the gap has been measured in capacitance-voltage (CV) and electron paramagnetic resonance measurements (EPR) to be amphoteric in nature with its maxima at 0.25 and $0.85 \mathrm{eV}$ above the valence band. 50

The second common defect is the $\mathrm{E}^{\prime}$ center. This defect in $\mathrm{SiO}_{2}$ is a binary structure: an oxygen vacancy with unpaired $\mathrm{Si}$ spin on one side, $\mathrm{O}_{3} \equiv \mathrm{Si}$ and a stripped positively charged ${ }^{+} \mathrm{Si} \equiv \mathrm{O}_{3}$ on the other side. The $\mathrm{E}^{\prime}$ center is a common occurrence in thermal oxide and is a possible source of oxide fixed-charge. ${ }^{\text {[5 }}$ It is characterized as a border trap, situated farther in the oxide than the $\mathrm{P}_{\mathrm{b}}$ center.

The presence of these defects play a significant part in the formation of quantum dots, and prevent quantum dot formation distorting the confinement potential and providing additional levels for tunneling. Chapters $⿴$ and $\mathbf{\square}$ go more into detail on this phenomenon.

Elimination of defects, such as the $\mathrm{P}_{\mathrm{b}}$ center, is commonly achieved through chemical passivation by introducing hydrogen in an annealing process. This process reduces the dangling bond spatial density from order $10^{13} \mathrm{~cm}^{-2}$ to $10^{10} \mathrm{~cm}^{-2}$ and improves numerous parameters related to MOSFET-like transistor operation and has also been shown to improve parameters related to quantum dot formation.

In order to create quantum dots reliably, it is thus necessary to perform this annealing step. For the heterostructures H1-H3 used in this work, the following applies: For H1, there is no passivation/annealing step. Annealing using a standard forming gas anneal of $\mathrm{H}_{2} / \mathrm{N}_{2}$ in this architecture was not possible due to dewetting, which is described in detail in Section B.3.D. The lack of annealing leads to single-hole tunneling through charge defects located underneath barriers in all cases. These results are described in Chapter Chapter $\mathbb{6}$.

Heterostructure 2, which introduces an ALD-grown $\mathrm{Al}_{2} \mathrm{O}_{3}$ layer, contains hydrogen as described in Section $\left[2 \cdot 2\right.$. The $\mathrm{Al}_{2} \mathrm{O}_{3}$ layer allows for annealing without dewetting. This process is shown to reduce charge defects to a substantial degree and is described in detail in Chapter $\mathbf{6}$.

Heterostructure 3 should show no appreciable difference at the $\mathrm{Si} / \mathrm{SiO}_{2}$ interface from $\mathrm{H} 2$, since only the gate-electrode material has been altered. There is the outside chance that the presence of $\mathrm{Al}$ improves annealing characteristics, in a process commonly known as alnealing.

Atomically flat/rough silicon As discussed in Section [3.2.3, the interface between silicon and $\mathrm{SiO}_{2}$ is not expected to be atomically flat but rather have a finite roughness. This is further exacerbated by the etching of silicon by BHF before high quality oxide growth, which is known to roughen the surface and leave $\mathrm{Si}\{110\}$ faceted nanoscale hillocks on the Si surface.

The roughness, with its characteristic length scale, causes the electric/chemical potential to fluctuate. If the length scale of the carrier wavefunction is comparable 
(a)

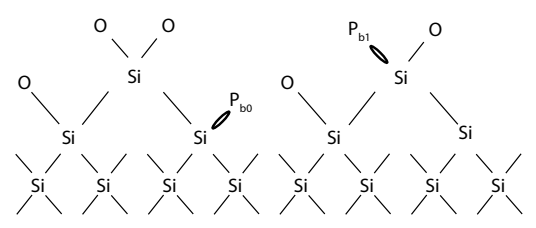

(b)

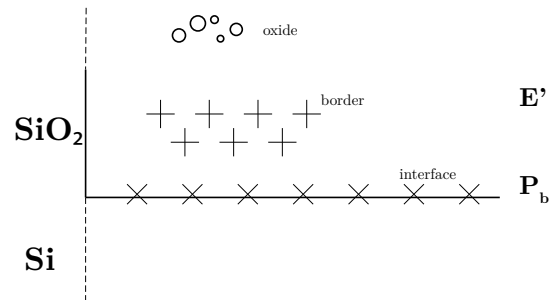

Figure 3.8: Defects at the $\mathrm{Si}$ (100) $\mathrm{SiO}_{2}$ interface. (a) shows two types of defect as the $\mathrm{Si} / \mathrm{SiO}_{2}$ interface, the $\mathrm{P}_{\mathrm{b} 0}$ and $\mathrm{P}_{\mathrm{b} 1}$ type dangling bond. (b) shows a categorization of defects based on their location relative to the interface. Interface defects are located exactly at the interface between $\mathrm{Si}$ and $\mathrm{SiO}_{2}$, border defects (such as the $\mathrm{E}^{\prime}$ center) some distance away, and oxide defects are located far away from the interface.

to the length scale of the roughness, this could lead to effects such as Anderson localization, a strong decrease in current in the device, $\stackrel{\square 6}{ }$ or unintentional spinflips. ${ }^{\square 7}$ Optimizing how abrupt the $\mathrm{Si} / \mathrm{SiO}_{2}$ interface is, would be a good candidate for improvement of quantum dot properties. As a means for spin-manipulation, the use of atomic steps has also been suggested.

Obtaining atomically flat silicon is possible by using using low-energy ion sputtering and a subsequent temperature step at $700{ }^{\circ} \mathrm{C}$.

Another method shown to work is to anneal the $\mathrm{Si}$ in ultrapure argon at $900^{\circ} \mathrm{C} . \overline{192}$ The surface is heated by Ar bombardment and recrystallizes in the lowest energy state. The key parameter in this process is the amount of trace oxygen, which in other cases etches the silicon and induces roughness. Among the reported improvements of this atomically flat $\mathrm{Si}$ have been the $1 / f$ noise characteristics of MOSFET structures. ${ }^{.22}$

Chemical means have also been attempted by etching in aqueous $\mathrm{NH}_{4} \mathrm{~F}$ (40\%), although atomics steps were not observed.

For all architectures in this work, this interface has been prepared in the same manner, and is expected not to be atomically flat.

\section{The $\mathrm{SiO}_{2} / \mathrm{Al}_{2} \mathrm{O}_{3}$ interface - fixed charge in $\mathrm{Al}_{2} \mathrm{O}_{3}$}

The negative fixed charge $Q_{f}$ in as-grown $\mathrm{Al}_{2} \mathrm{O}_{3}$ has been measured to be $10 \times 10^{11} \mathrm{~cm}^{-2} \amalg$ Subsequent annealing of this thermally grown layer increased $Q_{f}$ to a maximum of $10 \times 10^{13} \mathrm{~cm}^{-2}$, depending on the annealing temperature and ALD growth technique.

It has been proposed that the negative fixed charge is caused by a very thin interfacial layer that is off-stoichiometric ${ }^{\Gamma 20}$ and can be controlled by growing a preceding layer of $\mathrm{HfO}_{2}$ before the growth of the $\mathrm{Al}_{2} \mathrm{O}_{3}$. Figure B.yal shows 
schematically the location of the proposed fixed charge $Q_{f}$.

Samples without gate structures were grown with $5 \mathrm{~nm} 250{ }^{\circ} \mathrm{C} \mathrm{Al}_{2} \mathrm{O}_{3}$ layer and another layer of $5 \mathrm{~nm}$ grown at $100{ }^{\circ} \mathrm{C}$, and annealed at $400{ }^{\circ} \mathrm{C}$ in $\mathrm{H}_{2}$. These samples showed hole conduction on the order of $R_{S D}=50 \mathrm{k} \Omega$ at $T=4 \mathrm{~K}$. Would there be no fixed charge present, at $4 \mathrm{~K}$ all the charge carriers in the intrinsic silicon are frozen out and no conduction would be observed.

An interesting effect on the fixed charge was observed when exposing the samples to ozone in a UV-ozone reactor. Figure 3.96 shows the behaviour of the resistance of three devices with no gate electrodes deposited and only had the layers of $\mathrm{Al}_{2} \mathrm{O}_{3}$ grown. This resistance is shown as a function of cumulative time processed in an UV-ozone reactor. Startup transient effects such as the heating up of the Hg-lamp in this reactor has not been taken into account. An increase of conduction was observed when processing the samples in a UV-ozone reactor. This is proposed to be due to further population of the charge traps by carriers excited over the band gap by the UV photons. After this initial decrease in resistance it is seen that after approximately 10 minutes the resistance increases again. This increase can be explained by the oxidative effect of the ozone, which fills the oxide vacancies in the $\mathrm{Al}_{2} \mathrm{O}_{3}$.

\section{The $\mathrm{SiO}_{2} /$ Metal or $\mathrm{Al}_{2} \mathrm{O}_{3} /$ Metal interface}

To create the oxide/metal interface, metal is deposited by thermal or e-beam evaporation after lithography. In this process, the hot metal vapour is deposited at the colder substrate and interacts with said substrate. Ideally, the metal bonds to the substrate and adheres well enough to survive further process steps, such as lift-off. Should adhesion be a problem for the materials involved, an intermediate adhesion layer of a different material can be grown. These sticking layers are generally made of small radius atoms, such as Ti.

In our MOS heterostructures we are always dealing with metal/oxide interfaces. Before we continue let us briefly consider the stability of phases in compounds consisting of more than two constituents. In our case we are dealing with the oxide and the metal. For the $\mathrm{SiO}_{2} / \mathrm{Al}$ interface e.g. we are dealing with the three atomic species $\mathrm{Si}, \mathrm{Al}$, and $\mathrm{O}$. Which compound, given these three atomic species, is the most stable is dictated by the Gibbs free energy of the compounds.

The energies of the phases of various materials can be read out from an Ellingham diagram, which tabulates the Gibbs free energy versus temperature.

Given the mixture of $\mathrm{Si}, \mathrm{Al}$, and $\mathrm{O}$ for $\mathrm{H} 1$ at the metal/oxide interface, and the Gibbs free energies of the compounds $\mathrm{SiO}_{2}\left(\Delta G=-820 \mathrm{~kJ} \mathrm{~mol}^{-1}\right)$ and $\mathrm{Al}_{2} \mathrm{O}_{3}$ $\left(\Delta G=-1015 \mathrm{~kJ} \mathrm{~mol}^{-1}\right)$ at $T=200^{\circ} \mathrm{C}$, we expect that the most stable compound is the $\mathrm{Al}_{2} \mathrm{O}_{3}$. This implies that at the interface the $\mathrm{Al}$ is reduced by $\mathrm{O}$, which is consumed from the $\mathrm{SiO}_{2}$ layer, leaving elemental $\mathrm{Si}$ and $\mathrm{Al}_{2} \mathrm{O}_{3}$.

To explore this interface, we will now evaluate all three heterostructures on the basis of transmission electron microscopy (TEM) studies. 
(a)

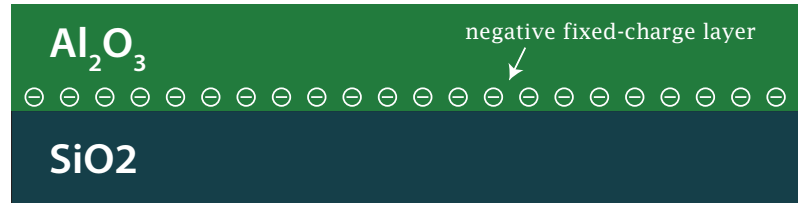

(b)

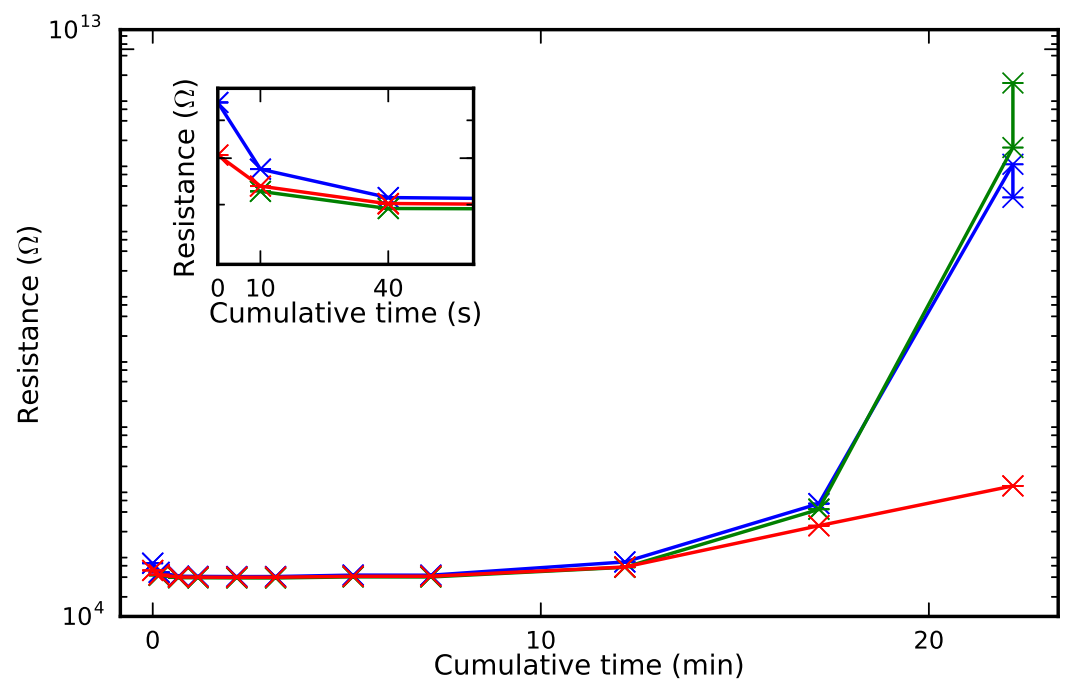

Figure 3.9: Fixed charge in $\mathrm{Al}_{2} \mathrm{O}_{3}$. (a) Schematic drawing of fixed charge, located at the border of the $\mathrm{Al}_{2} \mathrm{O}_{3} / \mathrm{SiO}_{2}$ interface. (b) Resistance of three samples as a function of time exposed in a UV-ozone reactor. Values were obtained by measuring the current-voltage relations $(T=4 \mathrm{~K})$ and extracting the linear resistance. Inset is a detailed view of the behaviour for small timescales

First, let us discuss H1, with its $\mathrm{SiO}_{2} / \mathrm{Al}$ interface, shown in Figure B.10a. An oxide layer can be seen surrounding the crystalline $\mathrm{Al}$ core. Given that the electrode has undergone the standard oxidation in ambient at $150{ }^{\circ} \mathrm{C}$ for 10 minutes, the oxide layer is indeed expected. However, would this step have been the only oxidation process, one would expect oxide formation only at parts of the electrode where exposed to ambient. The width of this interfacial layer is also too broad to have originated purely from oxidation from the side. The thickness of the interface is determined to be $d \approx 3 \mathrm{~nm}$.

The interface layer extends beyond the imaginary line of the $\mathrm{SiO}_{2}$ plane. This indicates that the $\mathrm{Al}$ has "eaten" into the $\mathrm{SiO}_{2}$ layer. Previous studies on the phase formed at this interface have concluded that a mixture of $\alpha$ and $\gamma$ phases are likely formed. These are less dense and have more "open" structures than the most stable $\alpha-\mathrm{Al}_{2} \mathrm{O}_{3}$.

Given that the formation of this interfacial layer could contain elemental Si 
and an undefined phase of $\mathrm{Al}_{2} \mathrm{O}_{3}$, it would be beneficial to eliminate the interfacial layer. Heterostructure 2 introduces an intermediate ALD-grown $\mathrm{Al}_{2} \mathrm{O}_{3}$ layer, changing the constituents of the oxide/metal interface as compared to heterostructure 1. It would be expected that the interface layer is reduced because the $\mathrm{Al}_{2} \mathrm{O}_{3}$ is stable and there is no reason for the $\mathrm{Al}$ to be oxidized.

Figure 3.100 shows a TEM image of this heterostructure. As compared to heterostructure 1 the same surrounding oxide layer is observed. However, the interfacial layer has instead increased to $d \approx 5 \mathrm{~nm}$. It could be that the $\mathrm{Al}_{2} \mathrm{O}_{3}$ underlayer is able to transport oxygen efficiently, or the electron affinity of $\mathrm{Al}$ allows for easier dissociation than for $\mathrm{SiO}_{2}$, combined with a diffusion process.

The third and final heterostructure supplants $\mathrm{Al}$ for $\mathrm{Pd}$, and utilizes the ALDgrown $\mathrm{Al}_{2} \mathrm{O}_{3}$ intermediate layer. It is immediately visible in Figure $3.0 \mathrm{C}$ that there is no more surrounding oxide layer. This could be since $\mathrm{Pd}$ knows no native oxide. The contrast of the $\mathrm{Pd}$ with the surrounding $\mathrm{Al}_{2} \mathrm{O}_{3}$ indicates that there is a negligible transition layer.

\subsubsection{Morphology}

In the end, quantum dot formation will be determined by the shape, size, and layout of the gate electrodes. Therefore, as a final category we will discuss the morphology of the heterostructure.

We will discuss some effects that relate to the morphology of the heterostructure. The shape of the structures as they interact with temperature and electric field, changing the potential landscape that influences quantum dot formation.

\section{Thermally induced non-uniform strain}

Strain is an important parameter in MOSFETs and has been used to manipulate

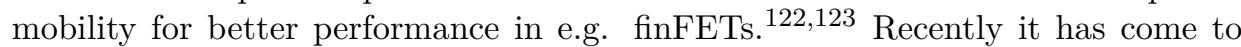
light that the morphology of the gate structure, together with differing thermal expansion coefficients, can lead to local strain in the heterostructure. ${ }^{\frac{83}{3}}$

The argument for this is as follows. Measurement of quantum dot devices is nearly always done at cryogenic temperatures. The expansion coefficient of each material is different, and thus each material contracts at a different rate. This resulting situation for a simple case of a metal gate electrode on a semiconductor material is sketched in Figure $\mathbf{B . T}$. At room temperature the materials can be considered nearly stress free, however when cooling down to cryogenic temperatures the metal will contract more than the substrate, causing compressive strain in the substrate. The strain modulates the $\mathrm{CB} / \mathrm{VB}$ on both ends of the metal gate, effecting a change in potential at these locations. The created potential confines carriers, and an unwanted quantum dot is created. Mitigating this effect, should one chose to, can be achieved by chosing metals with an expansion coefficient comparable to the substrate. In the case of $\mathrm{Si}$, poly-Si would be ideal. Alterna- 


\subsection{Defects in quantum dot heterostructures}

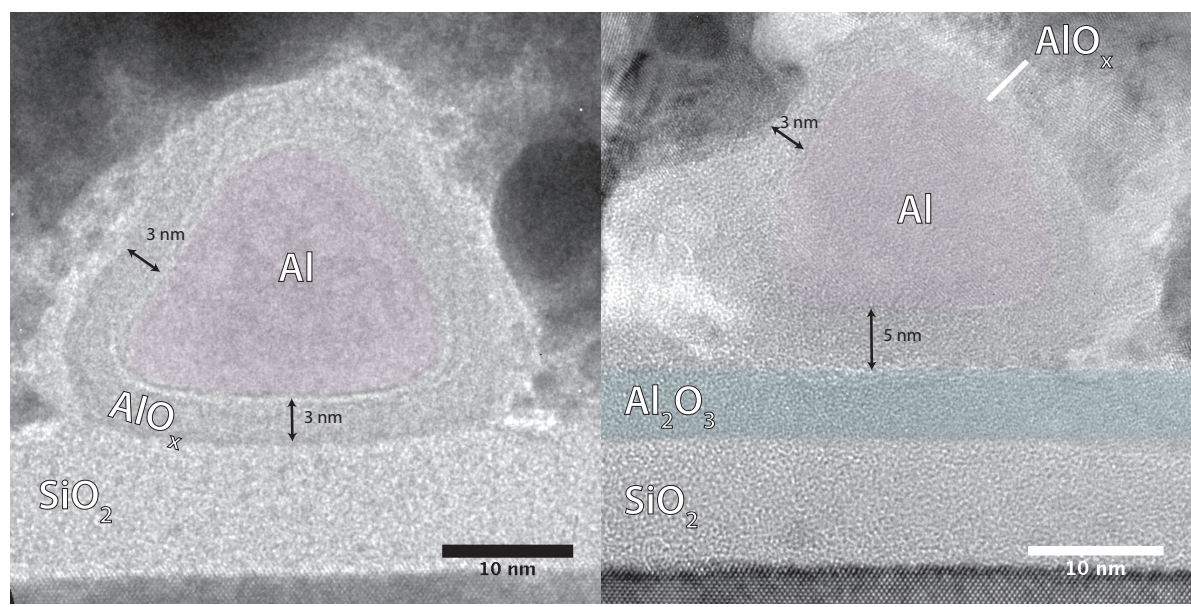

(H1)

$(\mathrm{H} 2)$

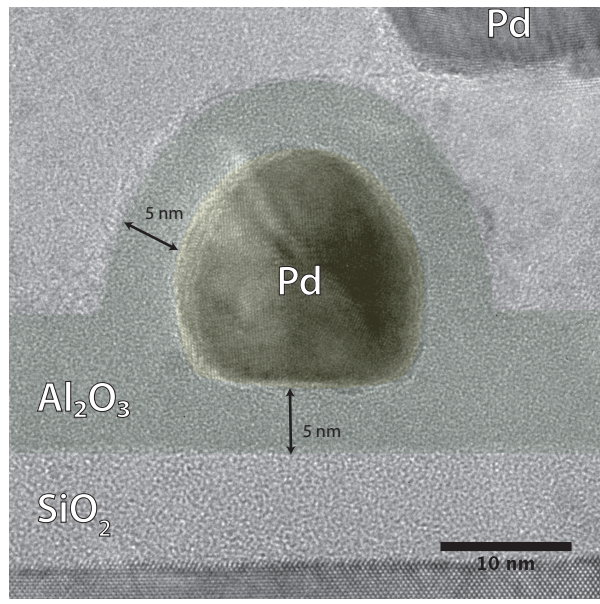

(H3)

Figure 3.10: HR-TEM images of all three heterostructures. (H1) Interaction of metal and oxide at the interface creates an oxide of undefined stoichiometry between the $\mathrm{Al}$ and $\mathrm{SiO}_{2}$. A slight curvature is observed where the $\mathrm{Al}$ "eats" into the $\mathrm{SiO}_{2}$. (H2) ALD-grown $\mathrm{Al}_{2} \mathrm{O}_{3}$, with the $\mathrm{Al}$ electrode evaporated to be 36 $\mathrm{nm}$ thick, followed by $\mathrm{Al} / \mathrm{Pd}(10 \mathrm{~nm} / 60 \mathrm{~nm}$ thick $)$. Interaction of $\mathrm{Al}$ with $\mathrm{Al}_{2} \mathrm{O}_{3}$ increases oxide layer directly under electrode to $5 \mathrm{~nm}$. (H3) No unexpected oxide layer observed. No contrast difference visible between ALD-grown $\mathrm{Al}_{2} \mathrm{O}_{3}$ grown at $100^{\circ} \mathrm{C}$ or $250{ }^{\circ} \mathrm{C}$. 


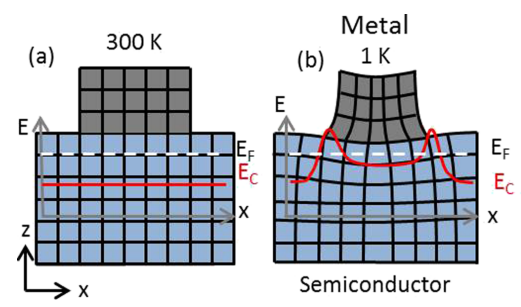

Figure 3.11: Strain causing bending of the CB/VB through a difference in thermal expansion coefficient of the constituent materials. From Thorbeck and Zimmerman $[8: 3]$.

tively, the distance to the substrate by adding more layers in between electrode and substrate could be increased. This would allow more relaxation of stress.

Let us again evaluate the consequences of this effect in our three heterotructures. Heterostructure 1 utilizes $\mathrm{Al}$ as a gate electrode material, which has one of the biggest thermal expansion coefficients. It is separated from the Si by only $8 \mathrm{~nm}$ of $\mathrm{SiO}_{2}$. One can thus expect that if the effect exists it will be biggest in this heterostructure.

Because the first gate electrode layer is separated by an additional $\mathrm{Al}_{2} \mathrm{O}_{3}$ layer in heterostructure 2, it is expected that strain is relieved more before reaching the Si. As discussed, mitigating the effect can also be accomplished by changing the electrode material. Pd has an expansion coefficient that is nearly twice as small as $\mathrm{Al}$. It is therefore expected that heterostructure 3 , which increases gateelectrode/Si separation by the $\mathrm{Al}_{2} \mathrm{O}_{3}$ layer and uses $\mathrm{Pd}$ as an electrode material, has the least local strain among all three heterostructures.

\section{Unintentional dielectric dots}

Another morphology effect, concerns the interaction with the electric field, and is also related to the formation of unintentional quantum dots.

In all heterostructures the first electrode layer is separated by a dielectric from the second electrode layer. This has the consequence that, when applying a voltage to the second covering electrode layer, the electric field gradient is smaller because it has to traverse more dielectric. This results in a potential that is slightly different at the sides of the first layer electrode. Figure $\mathbf{3 . 1 2}$ depicts this schematically with the lead gate having a "line of sight" to the semiconductor material. Along this line of sight the amount of dielectric material is larger. A simple simulation of this effect is shown in Figure [.13. Here the resulting potential is shown (1 nm below the $\mathrm{SiO}_{2}$ dielectric) as a function of difference in voltage on the lead and barrier gate. The resulting potential deviates from the ideal tunnel barrier potential on the order of $\mathrm{mV}$ when the voltage on the lead and barrier electrode is equal. This 
could lead to the formation of an unintentional quantum dot. When the voltage on the lead gate is increased, the ideal tunnel barrier profile is recovered.

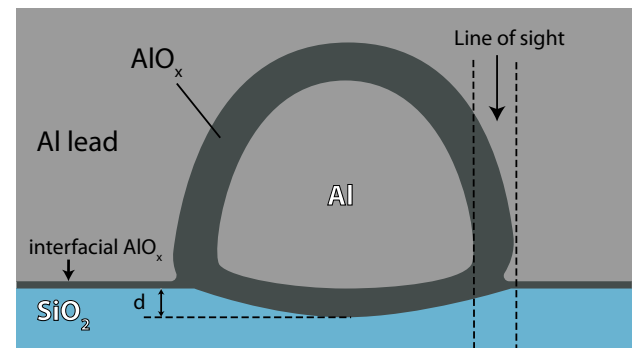

Figure 3.12: Schematic cross-section of barrier- and lead-electrode. The line of sight for electric field of the lead-gate is schematically indicated.

(a)

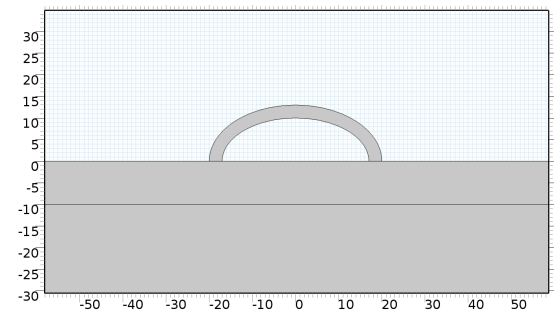

(b)

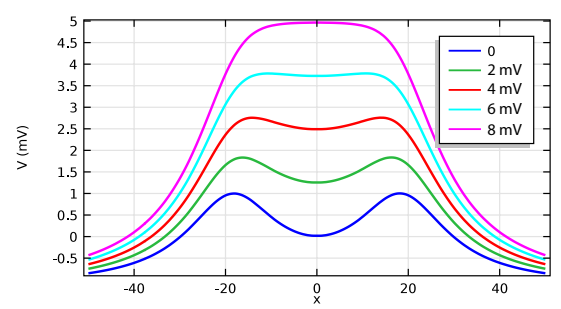

Figure 3.13: Simulation of a dielectric dot. (a) Geometry of simulation. (b) Attenuation of the local applied electric field by an increased volume of dielectric on either side of the gate electrode as a function of the difference in voltage on lead- and barrier-electrode.

\section{Grains in aluminum}

As discussed in Section [3.2, when evaporating thin films of metal an important parameter is the grain size of the resulting polycrystalline film. Especially when, in evaporating laterally constricted electrodes, the grain size becomes comparable to the width of the defined electrodes. This will result in an edge of the film which is rough along the line definition. An example of this for $\mathrm{Al}$ is given in Figure $3.15 \mathrm{~b}$. As in all other mechanisms described previously, the resulting potential will thus also be rough. It has been shown that roughness can lead to localization of a quantum dot. ${ }^{\text {ए24 }}$ Another mechanism which could be conceived is that the differently oriented strain in separate grains could could modulate potential via the thermally induced strain mechanism described previously. This is schematically depicted in Figure 3.15a. 
(a)

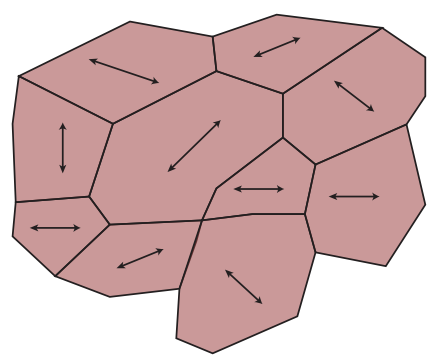

(b)

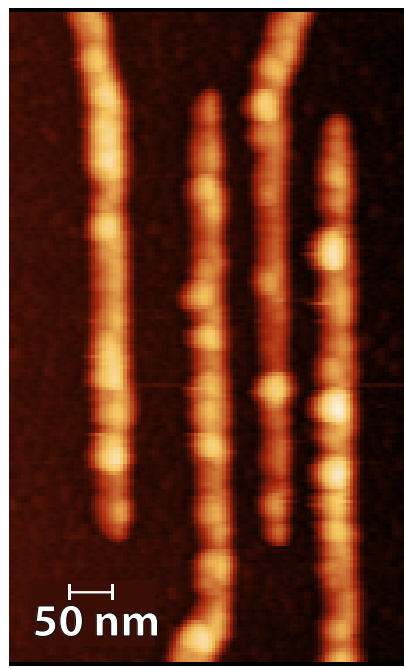

Figure 3.14: Grains in aluminum. (a) Depiction of the direction of temperatureinduced strain in a polycrystalline film. (b) AFM image of electrodes made from aluminum, electron-beam evaporated $36 \mathrm{~nm}$ thick. Grain-like features on the order of $50 \mathrm{~nm}$ are observed

\subsection{The annealing process}

Elimination and passivation of defects at the $\mathrm{Si} / \mathrm{SiO}_{2}$ interface is commonly achieved by annealing in a $\mathrm{H}_{2} / \mathrm{N}_{2}$ environment.

The passivation process for $\mathrm{P}_{\mathrm{b}}$ centers is governed, in the naive case, by

$$
\begin{gathered}
\mathrm{P}_{\mathrm{b}}+\mathrm{H}_{2} \stackrel{k_{a}}{\longrightarrow} \mathrm{P}_{\mathrm{b}} \mathrm{H}+\mathrm{H} \\
\mathrm{P}_{\mathrm{b}} \mathrm{H} \stackrel{k_{d}}{\longrightarrow} \mathrm{P}_{\mathrm{b}}+\mathrm{H}
\end{gathered}
$$

with associated activation energies $E_{a}=(1.5 \pm 0.4) \mathrm{eV}$ and $E_{d}=(2.83 \pm 0.03) \mathrm{eV}$. The rates of passivation $k_{a}$ and depassivation and $k_{d}$ are determined by temperature, through the Arrhenius expression $k \propto e^{-E_{a} / R T}$. The rates of the passivation and depassivation reaction lead to an equilibrium of the defect density. There is thus an optimum temperature at which the passivation process is at its most effective.

In this work we use ALD-grown $\mathrm{Al}_{2} \mathrm{O}_{3}$ to passivate our heterostructure. The process is done in two steps, with the growth of $\mathrm{Al}_{2} \mathrm{O}_{3}$ taking place at $T_{\text {dep }}$, and a separate annealing step done at $T_{\text {ann }}$.

The existence of hydrogen in ALD-grown oxide was shown through the use of

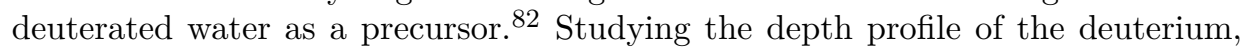
it was determined that the concentration in the $\mathrm{Al}_{2} \mathrm{O}_{3}$ was reduced, while the 
content of deuterium in the $\mathrm{SiO}_{2}$ and at the $\mathrm{Si} / \mathrm{SiO}_{2}$ interface showed a sharp increase, indicating the deuterium was being consumed in a passivation process.

The degree of passivation is determined by two factors. The first is the oxide growth temperature $T_{\text {dep }}$, which determines the density of the oxide, and its hydrogen content. The optimum $T_{\text {dep }}$ for passivation is reached when the effusion of hydrogen, controlled by the density of the oxide, is minimized, while the amount of hydrogen present is kept sufficient to passivate all dangling bonds in the annealing step that follows.

The second factor controlling the degree of passivation is the annealing temperature $T_{\text {ann }}$. Besides determining the final equilibrium defect density, $T_{\text {ann }}$ also determines how quickly this is reached. The best annealing temperature was previously determined to be $400^{\circ} \mathrm{C}$. ${ }^{126}$ Using this process, defect densities as low as $10 \times 10^{11} \mathrm{~cm}^{-2}$ have been reported.

The activation energy for passivation has been shown for ALD-grown $\mathrm{Al}_{2} \mathrm{O}_{3}$ to be $E_{a}=(1.2 \pm 0.5) \mathrm{eV}$

The ambipolar design of our heterostructures allows us to illustrate this process and its effects quite nicely in the effects on the threshold voltages $V_{T h}$. Shown in Figure 3.5 is the turn-on behaviour of a device with a heterostructure 2 design, with a single $\mathrm{Al}$ accumulation gate. The sample was measured once, before covering it in $\mathrm{ALD}$-grown $\mathrm{Al}_{2} \mathrm{O}_{3}$ with $\mathrm{T}_{\text {dep. }}$. It was then annealed at $\mathrm{T}_{\text {ann }} \approx 300{ }^{\circ} \mathrm{C}$ in Ar ambient for 45 minutes, and measured again.

It can be seen that $V_{T h}$ is reduced for both the hole and electron-operation regime after annealing. The absolute voltage decrease of $V_{T h}$ between the hole and electron-regime is related to the decrease in $\mathrm{P}_{\mathrm{b}}$ centers, which are amphoteric defects capable of storing both negative and positive charge. The layer of $\mathrm{P}_{\mathrm{b}}$ centers can thus be seen as an extra capacitor in series with the dielectric.

\subsection{Discussion and conclusion}

We have reviewed the many effects that can occur in heterostructures relating to quantum dot formation, and have identified effects pertaining to three categories: Materials, interfaces and morphology.

The planar quantum dot architecture first introduced by Angus et. al ${ }^{[20}(\mathrm{H} 1)$

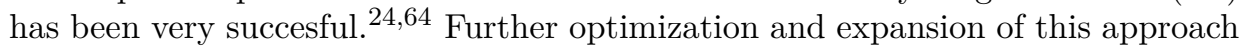
therefore bodes very well for the future. We have identified several areas where improvements can be made.

Firstly, we have observed that the ability to passivate defects at the $\mathrm{Si} / \mathrm{SiO}_{2}$ through annealing hinges primarily on the prevention of dewetting at higher temperatures. In this way, $\mathrm{H} 2$ improves upon the $\mathrm{H} 1$ design by introduction of an ALD-grown $\mathrm{Al}_{2} \mathrm{O}_{3}$ layer. This strikes two birds with one stone as it prevents dewetting, and enables the annealing of defects using (at least) the hydrogen present inside the grown film. 


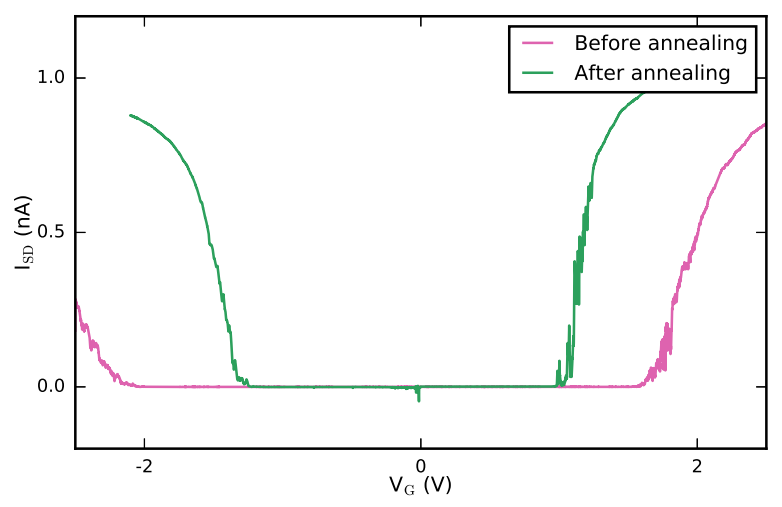

Figure 3.15: Current-voltage relation of a device as function of voltage on all electrodes $V_{G}$, both before and after annealing a device of the H1 heterostructure in the hole and electron operation regime. $V_{S D}=1 \mathrm{mV}, T \approx 4 \mathrm{~K}$

Unexpectedly however, an increase of the oxide layer directly beneath the $\mathrm{Al}$ electrode is observed. We postulate this might be due to an enhanced solubility of $\mathrm{Al}$ as compared to $\mathrm{Si}$, or an oxygen diffusion process, which would be more efficient in $\mathrm{Al}_{2} \mathrm{O}_{3}$. The slight curvature where the $\mathrm{SiO}_{2}$ has interacted with the $\mathrm{Al}$ as observed in $\mathrm{H} 1$, does not appear to be present in $\mathrm{H} 2$. H2 could therefore prevent the interfacial oxide between $\mathrm{Al}$ and $\mathrm{SiO}_{2}$ from forming, but this is inconclusive. The ability to anneal these devices now allows very well defined quantum dots up to $180 \mathrm{~nm}$ in length as shown in chapter 15 .

Supplanting Al by Pd as the electrode material in $\mathrm{H} 3$ does prevent the formation of a interfacial layer between the $\mathrm{Pd}$ and the $\mathrm{Al}_{2} \mathrm{O}_{3}$, as can been seen in TEM images. $\mathrm{Pd}$ would reduce temperature-induced strain, since its expansion coefficient is less than that of $\mathrm{Al}$. It is unclear how and if the polycrystalline nature of $\mathrm{Pd}$ differs from $\mathrm{Al}$, which could play a role in strain-related unintentional quantum dots.

A remaining issue is the fixed-charge present in the ALD-grown $\mathrm{Al}_{2} \mathrm{O}_{3}$. This can be a nuisance for quantum dot formation, since it induces a $2 \mathrm{DHG}$, even in areas where no electrodes are present. It could however also be used in creating depletion dots, as has been done for doped GaAs or SiGe heterostructures. For more on this, see Chapter 7 . First indications are that further processing of the devices in a UV ozone reactor eventually reduces the amount of fixed charge. Preliminary results have shown there to be little to no effect of the UV irradiation on the amount of passivated charge centers. Another avenue to reduce fixed charge could be to anneal at $300{ }^{\circ} \mathrm{C}$. This would reduce the fixed charge, but would also increase the final defect density $D_{i t}$.

Further optimization might include material such as poly-Si or TiN as an elec- 
trode material, which are amorphous materials, and therefore either have a nonuniform or reduced temperature-induced strain. The creation of an abrupt, atomically flat $\mathrm{Si} / \mathrm{SiO}_{2}$ interface could also improve the reliability of quantum dot formation. 



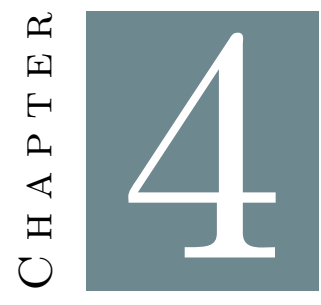

\section{Single-hole tunneling through a two-dimensional hole gas in intrinsic silicon}

In order for coherent operations to take place in a proposed quantum computer, ${ }^{\square 29}$ the quantum states of the corresponding qubits are required to be longlived. In the scheme proposed by Loss and DiVincenzo [1:2], quantum logic gates perform operations on coupled spin states of single electrons in neighboring quantum dots. Most experiments have focused on quantum dots formed in III-V semiconductors, especially GaAs; ${ }^{[38}$ however, electron spin coherence in those materials is limited by hyperfine interactions with nuclear spins and spin-orbit coupling. Group IV materials are believed to have long spin lifetimes because of weak spin-orbit interactions and the predominance of spin-zero nuclei. This prospect has stimulated significant experimental effort to isolate single charges in carbon

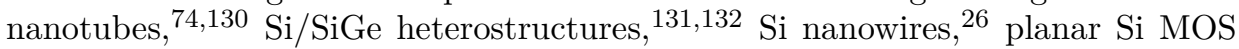
structures, ${ }^{64}$ and dopants in Si. ${ }^{\text {[3.3- } 13.5}$ Silicon not only holds promise for very long coherence times, ${ }^{\square]}$ but also for bringing scalability of quantum devices one step closer, and has thus attracted much attention for quantum computing purposes. $\underline{\text { [.5, } 1.36}$

The contents of this chapter have been previously published: P. C. Spruijtenburg et al., Single-hole tunneling through a two-dimensional hole gas in intrinsic silicon. Applied Physics Letters 102, 192105 (May 2013) 
Recently, coherent driven oscillations of individual electron and nuclear spins in silicon were reported. $\stackrel{\square 18, \square 37}{ }$ The spin resonance was magnetically driven by sending alternating currents through a nearby microwave line. A technologically more attractive way is electric-field induced electron spin resonance, as demonstrated in quantum dots made in GaAs/AlGaAs heterostructures, $138-140$ InAs nanowires, and InSb nanowires. ${ }^{\text {142 }}$ Electrical control of single spins requires mediation by either hyperfine or spin-orbit interaction. Although the latter is too weak for electrically driven spin resonance of electrons in silicon, the spin-orbit interaction for holes may well facilitate hole spin resonance by means of electric fields.

Up until now, single-hole spins have not yet been investigated in electrostatically defined silicon quantum dots. Here, we report on single-hole tunneling (SHT) in a gated silicon MOSFET nanostructure, based on an earlier n-type design by Angus et al. [20]. In this work we focus on low-temperature transport measurements through a two-dimensional hole gas (2DHG), which is electrostatically defined by a MOSFET-type architecture. To create the 2DHG we apply a negative potential to metallic gates on top of oxidized intrinsic silicon, raising the valence band to above the Fermi energy, thus allowing states to be occupied by holes. At $4 \mathrm{~K}$ we observe single-hole tunneling and control of the charge occupation in individual Coulomb islands. We are aware that similar results have simultaneously been obtained elsewhere. 14.3

\subsection{Fabrication}

Figure 1.0 shows an atomic force microscope image and a schematic cross section of the device structure, made with a combination of optical and electron-beam lithography (EBL), based on the recipe as described by Angus et al. [ZU]. Nearintrinsic silicon $\left(\rho \geq 10000 \Omega^{-\mathrm{cm}}\right)$, is used as the substrate. Source and drain regions are implanted with boron dopant atoms, which are activated by rapid thermal annealing, and serve as hole reservoirs. Ohmic contacts to these regions are made by sputtering Al-Si alloy (99:1) contact pads. A $10 \mathrm{~nm}$ thick high-quality $\mathrm{SiO}_{2}$ oxide window is thermally grown at $700^{\circ} \mathrm{C}$ and serves as an insulating barrier between the substrate and the aluminum gates. To remove charge traps and defects such as dangling bonds, the oxide is annealed in pure $\mathrm{H}_{2}$ at $400^{\circ} \mathrm{C}$ and a pressure of 10 mbar. Contact pads for gates are defined using optical lithography followed by development, evaporation of $\mathrm{Ti} / \mathrm{Pt}$, and subsequent lift-off. EBL is used to define the sub-micron aluminum gates, which will electrostatically control hole accumulation. Atomic force microscopy images show barrier gates with a typical width, height, and separation of $35 \mathrm{~nm}$, see figure t. Aa. After oxidation of the barrier gates, a second EBL step is used to define the lead gate. We have measured various devices and report here characteristic behavior of a representative sample. 
(a)

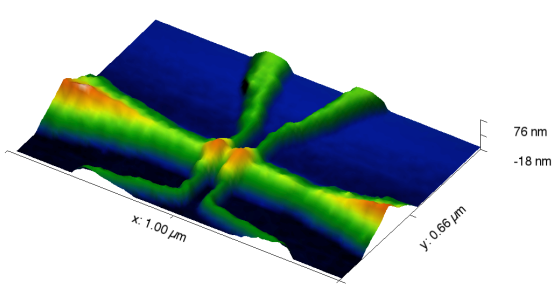

(b)

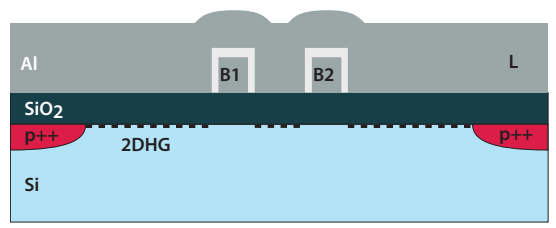

Figure 4.1: Si quantum dot gate structure. (a) Atomic force microscopy image of the device, showing the lead gate L horizontally across the image. The barrier gates B1 and B2 come in from the top center of the image. (b) A schematic crosssectional image of the device. The highly p-type doped source and drain regions are shown in the intrinsic silicon, on top of which is the $\mathrm{SiO}_{2}$ barrier. The $\mathrm{Al}$ gates are evaporated on top and electrically isolated from each other by aluminum oxide. The applied voltage on the aluminum gates creates a $2 \mathrm{DHG}$, indicated by the dotted lines.

\subsection{Holes}

To characterize hole transport in our devices we perform electrical transport measurements on samples submerged in liquid helium at a temperature of $T \approx 4.2 \mathrm{~K}$. Low-noise current amplifiers and voltage sources in combination with Pi-filters were used to characterize the devices. To measure a typical MOSFET turn-on characteristic, the same voltage $V_{G}$ is applied to all gates: $V_{L}=V_{B 1}=V_{B 2}$. Simultaneously, a bias voltage $V_{S D}$ is applied to the source and drain contacts. The gate voltages are then ramped to negative voltages while measuring the resulting current $I_{S D}$. Once the threshold voltage $V_{\mathrm{Th}}$ is reached, the valence band is pulled sufficiently above the Fermi energy so that hole states become available to be occupied. In the resulting $2 \mathrm{DHG}$, holes can then flow from source to drain and the device is 'turned on': during the sweeping of the gate voltages, the current increases up to roughly $1 \mathrm{nA}$, as shown in figure 4.2. The ability of the barrier gates to influence conduction in the $2 \mathrm{DHG}$ below the barriers is critical to operation of the devices. The barrier gate voltages $V_{B 1}$ and $V_{B 2}$ should be able to tune the corresponding potential barriers from highly transparent $\left(I_{S D} \geq 1 \mathrm{nA}\right)$ to opaque $(I \approx 0 \mathrm{nA})$. To test this, a 'pinch-off' curve is measured, by making the voltage on a barrier less negative whilst keeping the other gates well beyond the threshold voltage. Both barriers B1 and B2 can individually pinch off the conduction channel, and additionally show some resonances in the measured current. The results in Figure 42 shows the ability of each of the barriers to effectively control conduction in the channel.

Next, we measure the current versus both barrier gate voltages at constant bias and lead gate voltage (see Figure 1.3a). When the voltage applied to the barrier gates is too close to zero $\left(V_{B 1} \geq-2 \mathrm{~V}\right.$ or $\left.V_{B 2} \geq-2.6 \mathrm{~V}\right)$, the tunneling rate through the potential barriers becomes negligible. The current as a function of voltage 


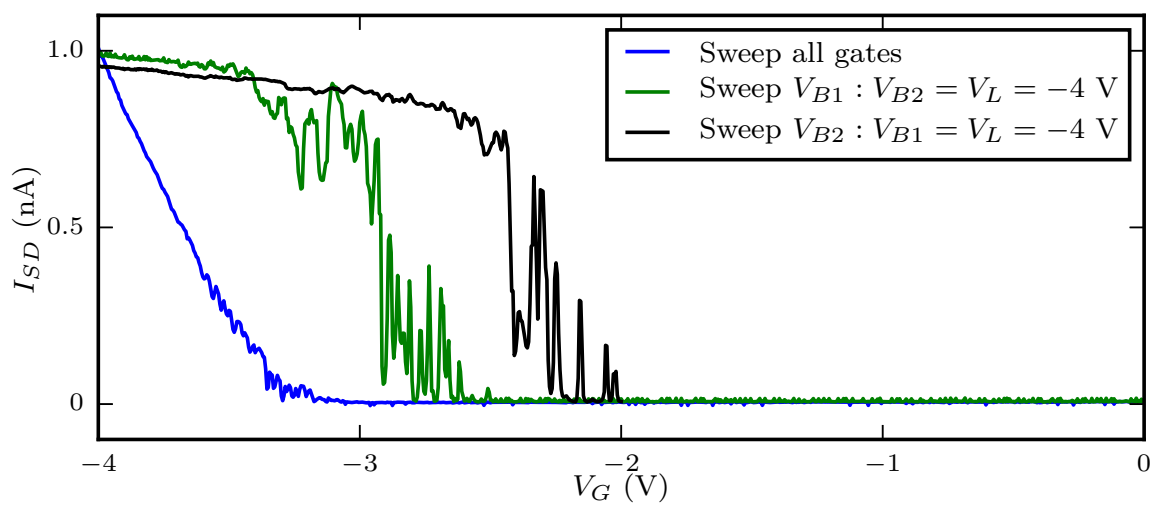

Figure 4.2: MOSFET type turn-on and pinch-off behavior at $T \approx 4.2 \mathrm{~K}$. A bias voltage of $V_{S D}=1 \mathrm{mV}$ is applied between source and drain contacts. For turn-on, one single voltage is applied to all the gates and increased. Pinch-off curves are measured by setting the voltage equal on all gates except the pinch-off barrier. The curves for turn-on, pinch-off with B1, and pinch-off with B2 are blue, green, and black, respectively.

applied to the barrier gates shows periodic resonances parallel to the axes of both $V_{B 1}$ and $V_{B 2}$. Resonances parallel to the axis of $V_{B 1}\left(V_{B 2}\right)$ are not influenced by a change in $V_{B 2}\left(V_{B 1}\right)$, indicating that those features are independently coupled to $V_{B 1}$ and $V_{B 2}$. We now focus on one of these resonances; specifically the one coupled most strongly to B2. To probe the features of this resonance, a constant $V_{S D}$ and $V_{B 1}$ is applied where $V_{B 1}$ is chosen such that the corresponding barrier is highly transparent. The subsequent measured source-drain current as a function of $V_{B 2}$ in figure $4.3 \mathrm{~b}$ shows periodic current peaks separated by regions of zero current. The sharp peaks correspond to Coulomb oscillations with regions of Coulomb blockade in between. Each time a peak is crossed, the occupation of the corresponding Coulomb island changes by one hole in a charge transition $N \leftrightarrow N+1$, where $\mathrm{N}$ is the number holes on the island. We can thus control the charge occupation of individual islands below the barrier gates. These islands are likely formed by disorder or roughness, e.g. impurities or charge traps in the $\mathrm{SiO}_{2}$. We conclude that figure $4.3 \mathrm{~b}$ show the trademark of single-hole tunneling and control of charge occupation in intrinsic silicon.

Energy spectroscopy was used to further characterize the device. The numerical differential conductance $d I / d V_{S D}$ with varying $V_{B 2}$ and $V_{S D}$ is suppressed periodically by Coulomb blockade, as shown in figure 4.4. The Coulomb diamonds are reasonably well defined and exhibit a single period, as evidenced by the parallel peaks in Fig. 4.3. The shape of the diamonds is most likely modulated by fluctuations in the conductance elsewhere in the device and the electrostatic environment, e.g. charge traps. At the diamond edges the electrochemical potential 
(a)

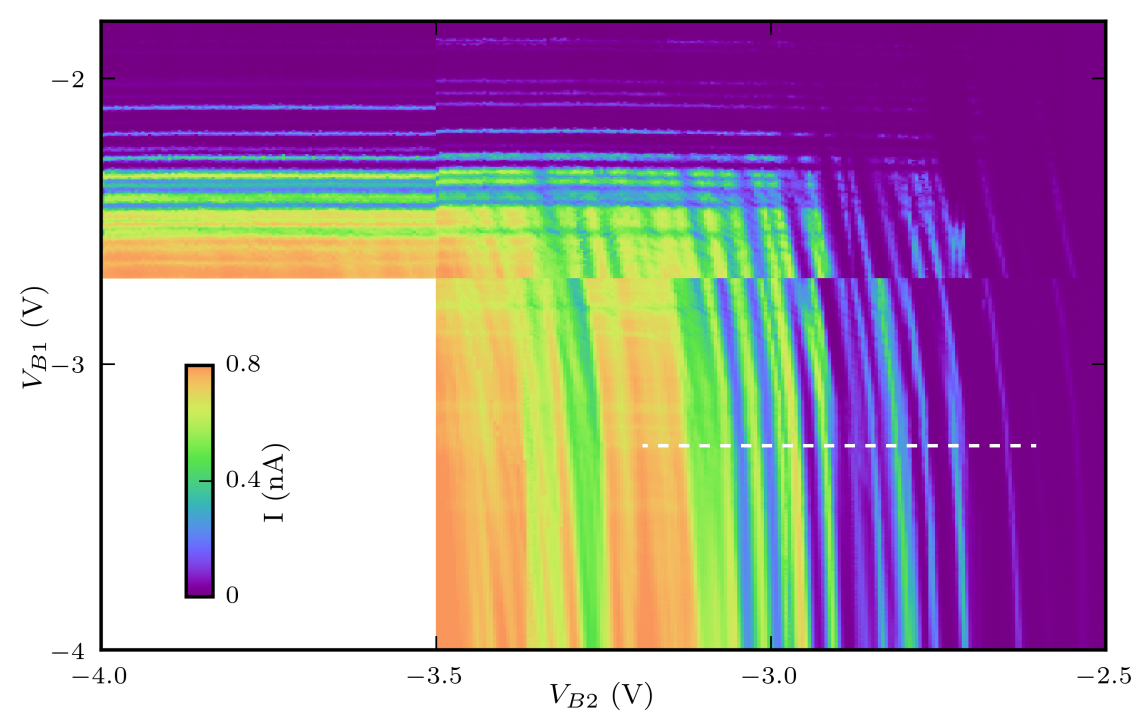

(b)

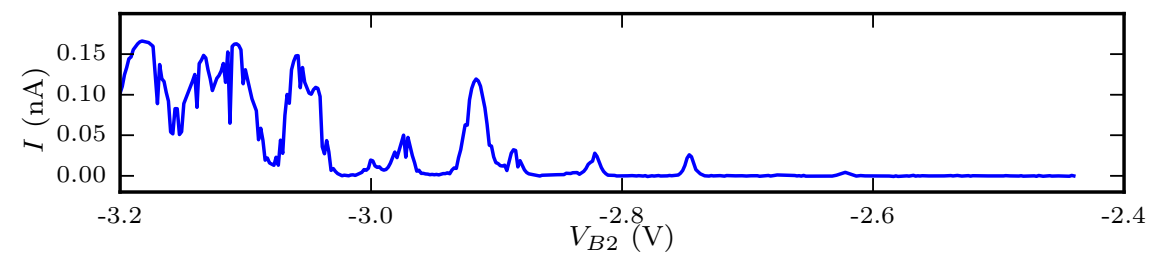

Figure 4.3: Single-hole tunneling in the linear transport regime. (a) The current as a function of applied barrier voltages $V_{B_{1}}, V_{B_{2}}$ with $V_{S D}=1 \mathrm{mV}$, showing periodicities in several directions. Three measurements at different times are visible, hence the discontinuity at $V_{B 1}=-2.7 \mathrm{~V}$ and $V_{B 2}=-3.5 \mathrm{~V}$. (b) Coulomb peaks in the current with varying $V_{B 2}$ at constant $V_{S D}=0.3 \mathrm{mV}, V_{L}=-3.95 \mathrm{~V}$, and $V_{B 1}=-3.1 \mathrm{~V}$

of the corresponding Coulomb island is resonant with either source or drain potential and single holes tunnel through the device. Most Coulomb diamonds close at zero source-drain bias, again indicating transport through a single island. $V_{B 2}$ changes the charge occupation of the island from $N$ to $N \pm 1$ by moving from one Coulomb diamond to the next. The Coulomb diamonds have very similar shapes across a wide voltage range and reproduce in repeated measurements, indicating the robustness of the device.

The charging energy $E_{C}$ of the island varies from $\sim 5$ to $10 \mathrm{meV}$. This corresponds to an island capacitance of $C \approx 32$ to $16 \mathrm{aF}$, which corresponds to a diameter of the island of about 38 to $76 \mathrm{~nm}$ in a classical disc capacitor model - where $E_{c}=e^{2} / 4 \epsilon_{0} \epsilon_{\mathrm{Si}} d$, with $\mathrm{d}$ the diameter of the island. In the last few diamonds, lines of increased conductance appear parallel to the diamond edges at positive and negative bias. We attribute this to resonant tunneling features as a results of extra 


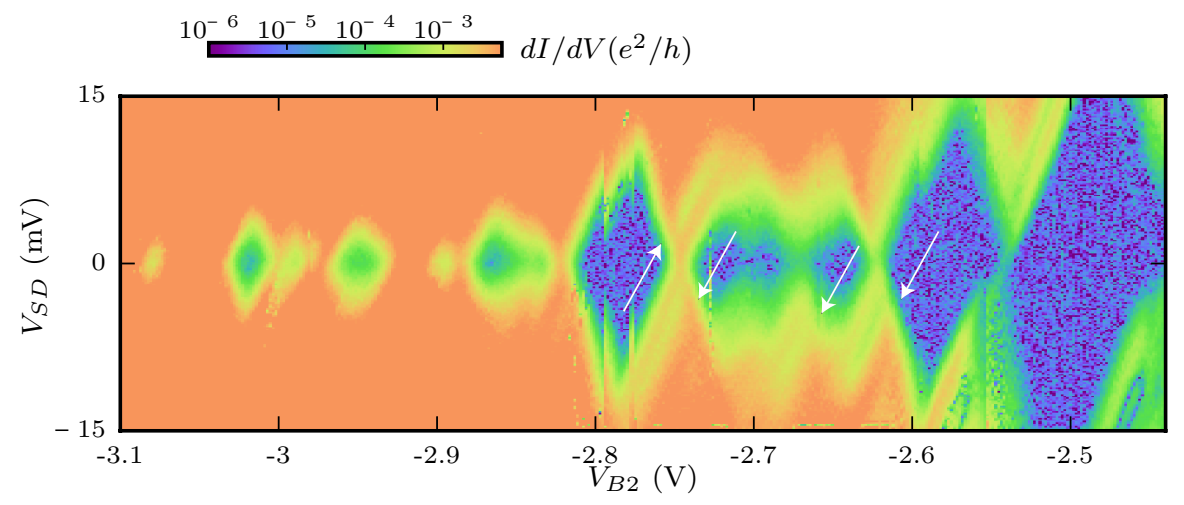

Figure 4.4: Single-hole tunneling in the non-linear transport regime. Bias spectroscopy taken at $V_{B_{1}}=-3.1 \mathrm{~V}$ and $V_{L}=-3.95 \mathrm{~V}$. Features of excited states are visible and are indicated by arrows.

available states for tunneling lined up with either source or drain. These features may correspond to orbital excited states, although the origin cannot be determined based on these data alone. ${ }^{144}$ The results in Fig. 4.4 shows single hole tunneling probed by energy spectroscopy, in which the present resonant tunneling features underline the ability to observe quantum states in these single-hole transistors.

\subsection{Discussion and conclusion}

To conclude, we have reported the fabrication and electronic characterization of p-type narrow-channel field-effect transistors in intrinsic silicon. Aluminum gate structures made on $\mathrm{Si} / \mathrm{SiO}_{2}$ with electron-beam lithography were used to create and control a two-dimensional hole gas at the interface of silicon and silicon oxide. Hole transport at $4 \mathrm{~K}$ can be controlled by barrier and lead gates, such that Coulomb peaks appear at small source-drain bias. Highly regular Coulomb peak lines and closing Coulomb diamonds in energy spectroscopy clearly indicate singlehole tunneling in the many-hole regime. The strong capacitive coupling to each respective barrier gate suggest that single Coulomb islands are created underneath or in the vicinity of the controlling gate. These islands are caused by local potential fluctuations due to e.g. impurities or charge traps in the $\mathrm{SiO}_{2}$ or at the interface of $\mathrm{Si}$ and $\mathrm{SiO}_{2}$. Silicon is known for being extremely sensitive to disorder, owing to the large effective mass of the charge carriers, which is even higher for holes than for electrons. The evidence for resonant tunneling features in energy spectroscopy indicates that these devices have demonstrable quantum confinement, even at relatively high temperatures. Further optimization of the fabrication process will focus on (i) improvement the material quality of metal, oxide and semiconductor, 
e.g. lowering the charge trap density in the $\mathrm{SiO}_{2}$ and reducing the grain size in the $\mathrm{Al}$, and (ii) minimization of disorder and roughness at the material interfaces, e.g. removing dangling bonds by introducing extra annealing steps. The resulting low-disorder hole quantum dots with tunable tunnel barriers pave the way towards control of single holes and single spins in silicon. 



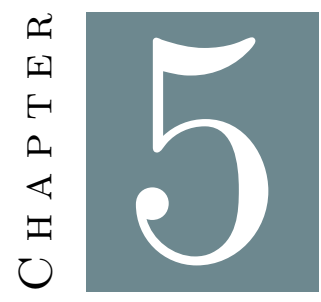

\title{
Passivation and characterization of charge defects in ambipolar silicon quantum dots
}

\begin{abstract}
Here, we show the role of charge defects in the context of the formation of electrostatically defined quantum dots. We introduce a barrier array structure to probe defects at multiple locations in a single device. We measure samples both before and after an annealing process which uses an $\mathrm{Al}_{2} \mathrm{O}_{3}$ overlayer, grown by atomic layer deposition. After passivation of the majority of charge defects with annealing we can electrostatically define hole quantum dots up to $180 \mathrm{~nm}$ in length. Our ambipolar structures reveal amphoteric charge defects that remain after annealing with charging energies of $\simeq 10 \mathrm{meV}$ in both the positive and negative charge state.
\end{abstract}

In order to perform sufficient coherent operations in a proposed quantum computer, ${ }^{\Gamma 29}$ the quantum states of the corresponding qubits are required to be longlived. In the scheme proposed by Loss and DiVincenzo, ${ }^{\text {ए] }}$ quantum logic gates

The contents of this chapter have been previously published: P. C. Spruijtenburg et al., Passivation and characterization of charge defects in ambipolar silicon quantum dots. Scientific Reports 6, 38127 (2016) 
perform operations on coupled spin states of single electrons in neighboring quantum dots (QDs). Most experiments have focused on quantum dots formed in III-V

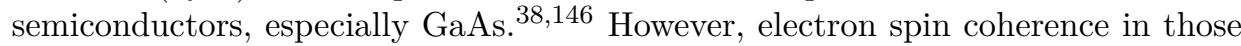
materials is limited by hyperfine interactions with nuclear spins and spin-orbit coupling. Group IV materials have been shown to have long spin lifetimes because of weak spin-orbit interactions and the predominance of spin-zero nuclei. This prospect has stimulated significant experimental effort to isolate single charges in carbon nanotubes, ${ }^{[4, \sqrt{47}} \mathrm{Si} / \mathrm{SiGe}$ heterostructures, ${ }^{\sqrt{48}, \sqrt{4.9}} \mathrm{Si}$ nanowires, ${ }^{[26}$ planar $\mathrm{Si}$

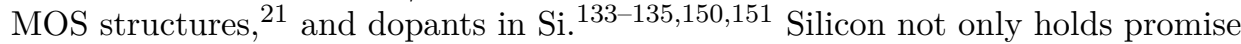
for very long coherence times, ${ }^{[27,23,24}$ but also for bringing scalability of quantum devices one step closer, and has thus attracted much attention for quantum computing purposes. ${ }^{65,[36}$ For the quantum states to be well-defined, it is necessary to define singular quantum dots. Disorder in the confinement potential can lead to unintentional quantum dots, ${ }^{[28, \pi 43}$ resonant tunneling features, ${ }^{[52, \pi 53}$ and Anderson localization. In silicon planar quantum dots, nanometer-size Coulomb islands are electrostatically defined in silicon in a gated MOSFET-type heterostructure. Apart from the quality of the crystalline silicon the quality and disorder in the heterostructure is directly linked to the disorder of the confinement potential.

In this chapter, we focus on disorder caused by defects at the $\mathrm{Si} / \mathrm{SiO}_{2}$ interface and investigate their effect on the formation of electrostatically defined quantum dots. We do this by creating an array of barrier electrodes, each capable of locally controlling the potential for quantum dot formation and probing the respective crystalline environment. We show that an annealing process with $\mathrm{Al}_{2} \mathrm{O}_{3}$, grown by atomic layer deposition (ALD), reduces the disorder such that quantum dots $180 \mathrm{~nm}$ in length can be formed. Furthermore, we observe that after annealing a small percentage of barriers show charge transitions with an amphoteric character.

The role of defects at the $\mathrm{Si} / \mathrm{SiO}_{2}$ interface in this heterostructure has been extensively studied in the context of MOSFET technology. One of the most studied

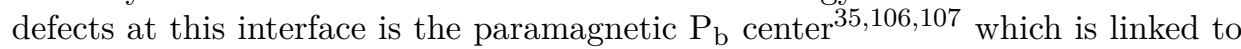
surface charges, decreased mobility, and the negative-bias temperature instability (NBTI) effect. $108, \square 0$ For quantum computation, $\mathrm{P}_{\mathrm{b}}$ centers have been used in coherent manipulation of spin-dependent charge-carrier recombination of phosphorus donors. ${ }^{110}$ Defects at the interface have also been associated with random telegraph signals at low temperatures and characterized by means of single-electron

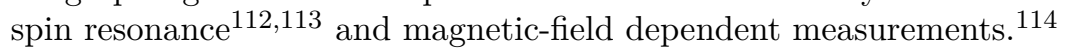

The $\mathrm{P}_{\mathrm{b}}$ center is in essence a $\mathrm{Si}$ atom with a dangling bond that has formed due to the incommensurability of the crystalline $\mathrm{Si}$ and the amorphous $\mathrm{SiO}_{2}$. Two types of $\mathrm{P}_{\mathrm{b}}$ center can be further distinguished. The $\mathrm{P}_{\mathrm{b} 0}$ center is backbonded to two Si atoms and one oxygen atom, while the $\mathrm{P}_{\mathrm{b} 1}$ center is backbonded to three $\mathrm{Si}$ atoms. For the $\mathrm{P}_{\mathrm{b} 0}$ center, the density of interface states $\mathrm{D}_{\mathrm{it}}$ in the gap is amphoteric in nature with its maxima at 0.25 and $0.85 \mathrm{eV}$ above the valence band. $\stackrel{5}{ }$ Passivating $\mathrm{P}_{\mathrm{b}}$ centers is commonly achieved by introducing hydrogen to the dangling bonds in an annealing process. This process reduces the dangling bond spatial density from order $10^{13} \mathrm{~cm}^{-2}$ to $10^{10} \mathrm{~cm}^{-2}$ and improves numerous 
parameters related to MOSFET-like transistor operation and has also been shown to improve parameters related to quantum dot formation. $[20,55$

Addressing only a single quantum dot defined by electrostatic gating can be hampered by a charge defect. If a charge defect is located beneath an electrode intended to form a tunnel barrier, this electrode acts a gate on this defect. Instead of forming a single tunnel barrier to the $\mathrm{QD}$, the levels of the defect remain available for transport resulting in additional single-charge tunneling events. This situation is sketched for an unpassivated charge defect in Figure 5. I for the right tunnel barrier.

While the valence band of the right tunnel barrier is below the Fermi level, the level of the charge defect is above the Fermi level and is thus available for hole transport. If the defect is in close enough proximity to the QD, an ill-defined double quantum dot can form. If the defect is passivated by hydrogen, the potential is smooth and behaves as a normal tunnel barrier for the QD. The same arguments hold for an impurity level just below the conduction band when measuring an electron QD.

\subsection{Device structure and fabrication}

Figure 5.16 shows an atomic force microscope image and Figure 5.5 a schematic cross section of our device structure, made with a combination of optical and electron-beam lithography (EBL), based on the recipe as described by Angus et al. ${ }^{20}$ We further distinguish two device types - A and B - specified below. Nearintrinsic $(100)$ silicon $(\rho \geq 10 \mathrm{k} \Omega \cdot \mathrm{cm})$ is used as the substrate. Source and drain regions are implanted with boron/phosphorus dopant atoms, which are activated by rapid thermal annealing, and serve as hole/electron reservoirs. Ohmic contacts to these regions are made by sputtering Al-Si alloy (99:1) contact pads. A $10 \mathrm{~nm}$ thick high-quality $\mathrm{SiO}_{2}$ oxide window is thermally grown at $900^{\circ} \mathrm{C}$ and serves as an insulating barrier between the substrate and the aluminum gates. Contact pads for gates are defined using optical lithography followed by development, evaporation of $\mathrm{Ti} / \mathrm{Pd}$, and subsequent lift-off. At this point, for device type $\mathrm{B}$, a $5 \mathrm{~nm}$ $\mathrm{Al}_{2} \mathrm{O}_{3}$ "underlayer" using $\mathrm{ALD}$ at a process temperature of $250^{\circ} \mathrm{C}$ is grown on top of the $\mathrm{SiO}_{2}$. Subsequently, EBL followed by evaporation and liftoff is used to define the sub-micron aluminum gates. These gates will electrostatically control charge accumulation. In the first step, the barrier gates are created and thermally oxidized. In the second step, the lead gate is created. Atomic force microscopy images show barrier gates with a typical width of $35 \mathrm{~nm}$, and a minimum separation of $180 \mathrm{~nm}$, while the lead gate has a width of approximately $80 \mathrm{~nm}$, see Figure 5.10. Following the creation of the aluminum gate structures, we perform a first set of measurements. Then we grow a final "overlayer" of $5 \mathrm{~nm} \mathrm{Al}_{2} \mathrm{O}_{3}$ at $100^{\circ} \mathrm{C}$, and the sample is heated to $300^{\circ} \mathrm{C}$ for 45 minutes in pure Ar ambient of 10 mbar. As a final step, the sample is cleaned in UV ozone for 2 minutes to remove any moisture present. 
(a)

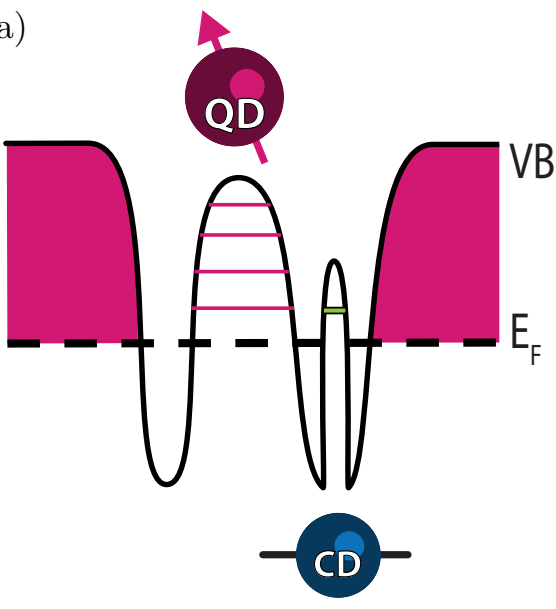

(c)

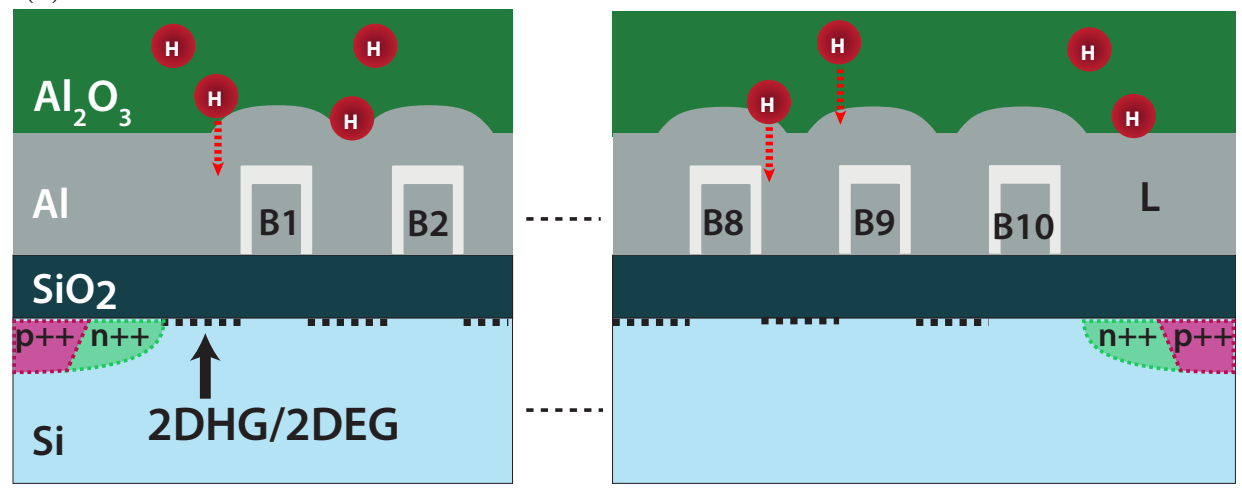

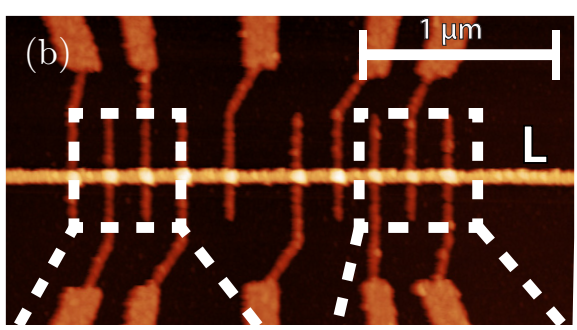
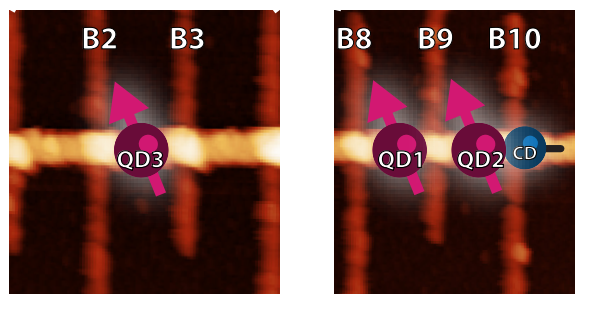

Figure 5.1: Si barrier array device structure. (a) Electrochemical potential profile of a hole quantum dot with and without a passivated and unpassivated charge defect in the left and right tunnel barrier respectively. In case of an unpassivated charge defect ("CD"), an extra level in the bandgap (green) is available for transport. (b) Atomic force microscopy image of the device, showing the lead gate L horizontally across the image. The barriers B1 through B10 allow local probing of the $2 \mathrm{DHG} / 2 \mathrm{DEG}$ at many locations. (c) A schematic cross-sectional image of the device. The hydrogen $(\mathrm{H})$ present in the $\mathrm{Al}_{2} \mathrm{O}_{3}$ can passivate charge defects during annealing. The applied voltage on the aluminum gates creates a $2 \mathrm{DHG} / 2 \mathrm{DEG}$, indicated by the dashed lines. 
To study both defects and their influence on QD formation, we use a twofold approach: by using an array of electrodes (seen in an AFM image in Figure 5.16), each acting as a single barrier, we can locally probe the $2 \mathrm{D}$ carrier gas and observe single-charge tunneling events. At the same time, using an ambipolar device structure $\sqrt{[3.5 .5}$ it is possible to infer if accessible levels are located near the conduction or valence band edge by either inducing a $2 \mathrm{D}$ hole gas (2DHG) or $2 \mathrm{D}$ electron gas (2DEG). Thus, our ambipolar structures allow investigation of both the hole and electron nature of these defects.

\section{2 properties of $\mathrm{Al}_{2} \mathrm{O}_{3}$ grown by atomic layer deposi- tion}

Recent work has shown that $\mathrm{Al}_{2} \mathrm{O}_{3}$ grown with ALD contains hydrogen and can be used for passivation. We use this property of $\mathrm{ALD}$-grown $\mathrm{Al}_{2} \mathrm{O}_{3}$ in a separate anneal step after $\mathrm{Al}_{2} \mathrm{O}_{3}$ deposition. We further postulate that the mechanical stability of our gate structure with pure $\mathrm{Al}$ is improved by encapsulation in this $\mathrm{Al}_{2} \mathrm{O}_{3}$ oxide. It is energetically more favorable to form $\mathrm{Al}_{2} \mathrm{O}_{3}$ than $\mathrm{SiO}_{2}$ because the Gibbs free energy change of $\mathrm{Al}_{2} \mathrm{O}_{3}$ formation is lower than that of $\mathrm{SiO}_{2}$. $\mathrm{Al}$ is known to reduce $\mathrm{SiO}_{2}$ as confirmed by transmission electron microscopy of our samples. ${ }^{[\pi /}$ It has also been shown that $\mathrm{Al}$ can spike through $\mathrm{SiO}_{2}$ causing leakages and shorts to the channel. Diff Diffusivity of $\mathrm{Al}$ at temperatures around $300^{\circ} \mathrm{C}$ can cause dewetting, ${ }^{98}$ or void formation can occur due to different thermal expansion coefficients.

We have found that the overlayer of $5 \mathrm{~nm}$ of $\mathrm{Al}_{2} \mathrm{O}_{3}$ alone in device type $\mathrm{A}$ stabilizes the pure aluminum electrodes. Without this overlayer void formation and dewetting-like behaviour was observed in all devices after the $300^{\circ} \mathrm{C}$ annealing step (see Supplementary Information). The presence of an $\mathrm{Al}_{2} \mathrm{O}_{3}$ underlayer in device type $\mathrm{B}$, between the $\mathrm{SiO}_{2}$ and the $\mathrm{Al}$ gate structure could prevent the reduction reaction that would otherwise occur at the $\mathrm{Al} / \mathrm{SiO}_{2}$ interface. The under- and overlayer of $\mathrm{Al}_{2} \mathrm{O}_{3}$ respectively change the surface energy and suppress surface diffusivity of the $\mathrm{Al}$ film.

\subsection{Quantum dot formation before and after annealing}

We have measured various devices and report here characteristic behaviour of two types of samples A and B. First, we will focus on device A and the influence of charge defects on the formation of electrostatically defined QDs. Device A (shown in Figures $5.1 \mathrm{~b}$ and 5.1d) does not contain a layer of $\mathrm{Al}_{2} \mathrm{O}_{3}$ between the $\mathrm{SiO}_{2}$ and the $\mathrm{Al}$ gate structures. For its transport characteristics we will focus on hole transport. Device $\mathrm{A}$ is measured once before $\mathrm{Al}_{2} \mathrm{O}_{3}$ overlayer growth and subsequent annealing step. After this passivation procedure the device is measured 

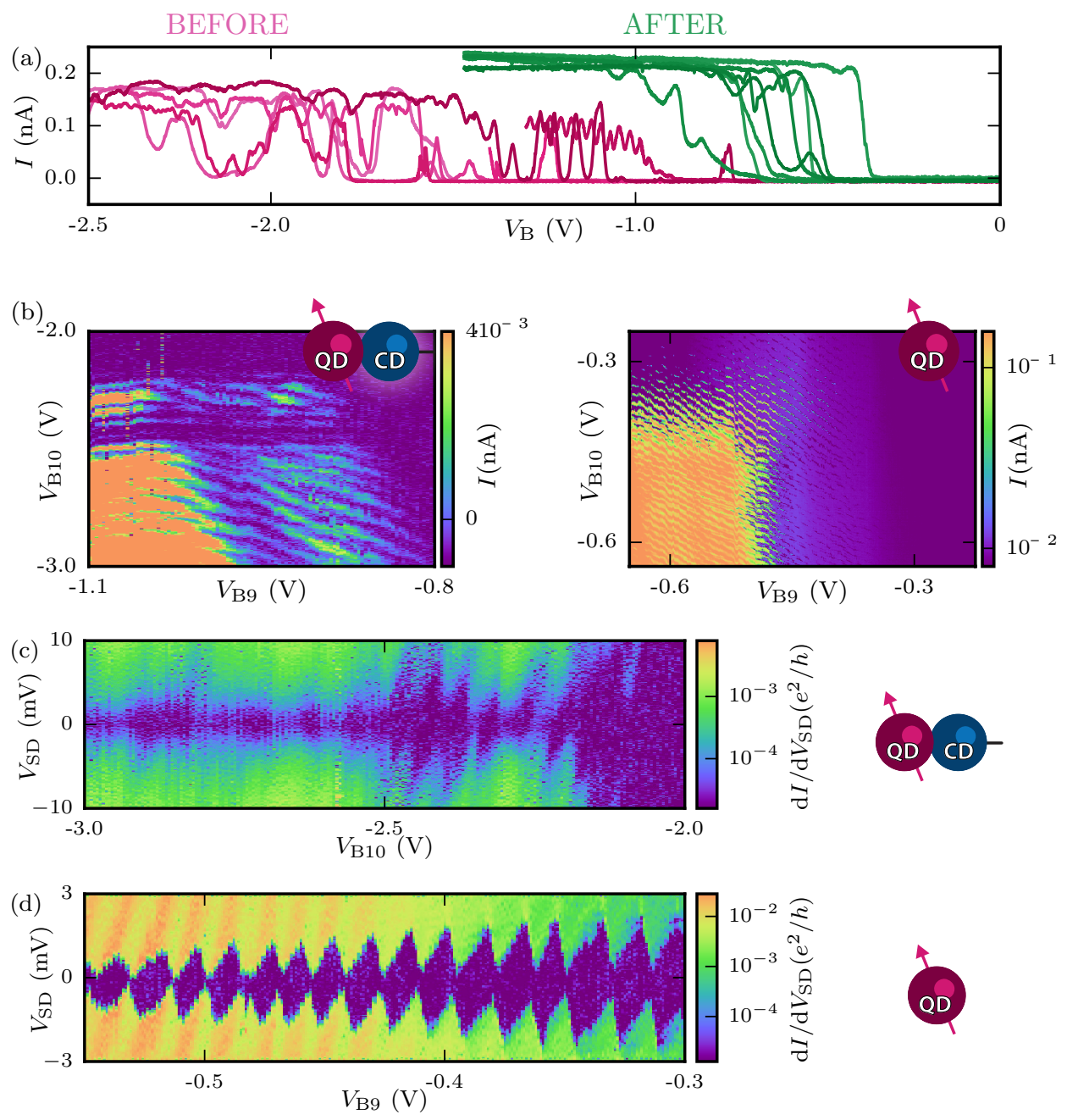

Figure 5.2: Transport measurements of device A before and after annealing. (a) Current-voltage relation at $T \simeq 4.2 \mathrm{~K}$ before (red) and after (green) annealing. Individual barriers are swept to $0 \mathrm{~V}$ while all other gate voltages are maintained above the threshold voltage with $V_{\mathrm{SD}}=1 \mathrm{mV}$ and $V_{\mathrm{L}}=-4.6 \mathrm{~V}$ before, and $V_{\mathrm{L}}=$ $-2.4 \mathrm{~V}$ after anneal. (b) Linear transport measurements of current $I$ as a function of barrier gate voltages $V_{\mathrm{B} 10}$ and $V_{\mathrm{B} 9}$ before (left) and after (b) (right) annealing. (d) The differential conductance $d I / d V_{\mathrm{SD}}$, as a function of source-drain bias $V_{S D}$ and barrier gate voltage $V_{B 10}$ before annealing, taken at $V_{\mathrm{B} 9}=-950 \mathrm{mV}$. Irregular Coulomb-diamond like features are visible with charging energies up to $10 \mathrm{meV}$. (e) The same measurement after annealing with $V_{\mathrm{B} 10}=-350 \mathrm{mV}$ and $T \simeq 10 \mathrm{mK}$. Well-defined Coulomb diamonds with charging energies of $2-3 \mathrm{meV}$ are visible, corresponding to an electrostatically defined Coulomb island $180 \mathrm{~nm}$ in length and $80 \mathrm{~nm}$ in width. 


\subsection{Quantum dot formation before and after annealing}

again. The current $I$ is measured at the drain while the drain is kept at ground. Additionally, all voltages are given with respect to ground.

Figure $5.2 \mathrm{~d}$ shows the current-voltage relation of the individual barriers before and after annealing. For these measurements all electrodes except the barrier electrode in question are at sufficiently high voltage to allow conduction, while one of the barrier electrodes is swept to such a voltage that the barrier will become opaque and thus "cut off" the channel locally. Cut-off voltages decrease from a spread of $-2 \mathrm{~V}$ to $-1 \mathrm{~V}$ to a more narrow region of $-1 \mathrm{~V}$ to $-0.5 \mathrm{~V}$. This is expected to be due to the reduction of a charge layer formed by charge defects present before annealing. The decreased number of resonances in the current $I$ indicates disorder in the device has decreased substantially after annealing. The threshold voltage $V_{\mathrm{Th}}$, here defined as the voltage at which the entire channel becomes conducting when applying the same voltage to all gates, is also lowered after annealing from $V_{\mathrm{Th}} \simeq-4 \mathrm{~V}$ to $V_{\mathrm{Th}} \simeq-2.3 \mathrm{~V}$ (not shown).

We have observed that nearly all resonances associated with the barriers disappear after $\mathrm{Al}_{2} \mathrm{O}_{3}$ growth and the subsequent annealing procedure. We have confirmed this in separate bias spectroscopy measurements where Coulomb diamonds of order $E_{C} \simeq 10 \mathrm{meV}$ coupled exclusively to one barrier gate are no longer present after annealing.

Before annealing, Figure $5.2 b$ (left) contains at least two sets of parallel lines of Coulombs peaks with distinct coupling to B9 and B10. One set of nearly horizontal lines runs from $-2.4 \mathrm{~V}$ to $-2.3 \mathrm{~V}$ parallel to the $V_{B 9}$ axis. We ascribe these horizontal lines to a charge defect underneath B10. ${ }^{\llbracket 28}$ Another feature is seen in the increased tunnel current running vertically around $V_{\mathrm{B} 9}=-900 \mathrm{mV}$, indicating another feature coupled strongly to B9 with different periodicity from the one underneath B10. A set of irregular diagonal lines is also visible. Thus, this feature is coupled to both B9 and B10, signifying a quantum dot centered between B9 and B10. However, these lines are interrupted and not continuous when intersecting the features associated with the defects, which implies a QD in series with at least 2 defects. After annealing the same device shows very ordered linear transport data in Figure $5.2 \mathrm{~d}$ (right) $(T \simeq 10 \mathrm{mK})$ at $V_{\mathrm{SD}}=0.3 \mathrm{mV}$ with uninterrupted diagonal lines equally coupled to $\mathrm{B} 9, \mathrm{~B} 10$. We extract ${ }^{{ }_{\mathrm{B} 10}} /_{\mathrm{C}_{\mathrm{B} 9}} \simeq 1$, where $C_{\mathrm{B}_{i}}$ is the capacitance of barrier gate $\mathrm{i}$ to the dot. The absence of a charge sensor does not allow us to probe how many electrons are on the dot but the amount of visible charge transitions $(\simeq 50)$ and the lack of interruptions in the charge transition lines indicates the dot has a very controllable charge occupation over a large range of voltages.

We next perform bias spectroscopy in order to more accurately determine the ability to form quantum dots. A bias spectroscopy before annealing reveals several overlapping sets of Coulomb diamonds, see Figure 5.2d. This is indicative of charge centers in series with the QD. Coulomb diamonds with a charging energy of $E_{C} \simeq 10 \mathrm{meV}$ are visible as well as another set of diamonds with a charging energy 3-5 meV . After annealing, Figure $5.2 \mathrm{e}$ shows very regular Coulomb diamonds with a 
well-defined charging energy of $E_{C} \simeq 2 \mathrm{meV}$. Below $V_{\mathrm{B} 9}=-400 \mathrm{mV}$ the diamonds are closing. Around $V_{\mathrm{B} 9}=-400 \mathrm{mV}$ a band of Coulomb blockade, with $\left|V_{\mathrm{SD}}\right|<0.3 \mathrm{mV}$, causes the diamonds not to close. This additional blockade is not due to the dot itself but rather from blockade present further along the 3 micron long channel formed by the lead gate which we were unable to mitigate. The many charge transitions and regular spacing of the diamonds indicates the low level of disorder in the device.

(a)
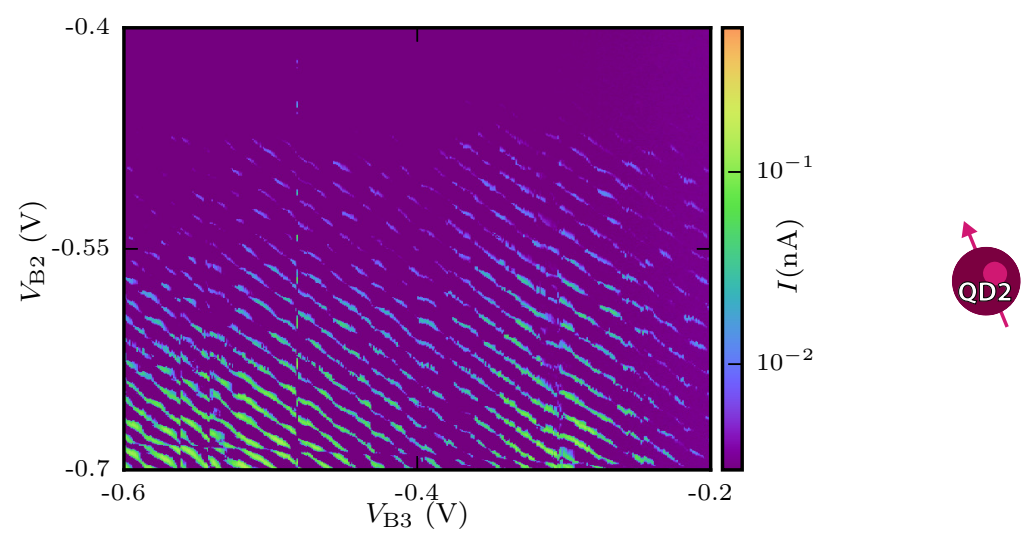

(b)
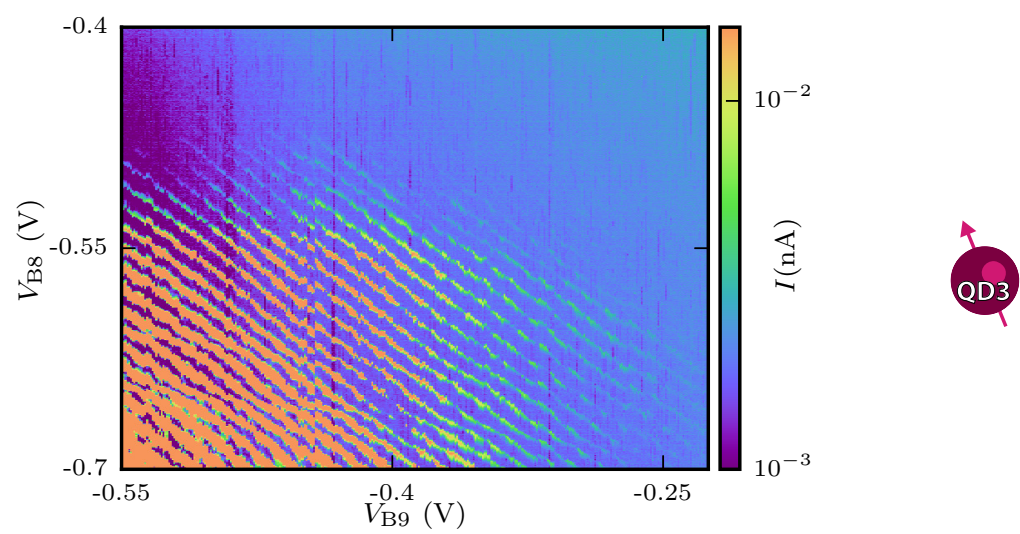

Figure 5.3: Intentional quantum dots at both ends of device A. Linear transport measurements of current $I$ as a function of adjacent barrier gate voltages. An intentional quantum dot created (a) between B2 and B3 with $V_{\mathrm{L}}=-2.9 \mathrm{~V}, V_{\mathrm{SD}}=$ $0.5 \mathrm{mV}$ and (b) between B8 and B9 with $V_{\mathrm{L}}=-3.1 \mathrm{~V}, V_{\mathrm{SD}}=1 \mathrm{mV}$. Diagonal current peaks indicate charge transitions coupled almost equally to both B2, B3 $\left({ }^{C_{\mathrm{B}}} / \mathrm{C}_{\mathrm{B} 3} \simeq\right.$ 0.75) and B8, B9 $\left({ }^{{ }^{\mathrm{B} 8}} / C_{\mathrm{B} 9} \simeq 0.95\right)$.

Figure 5.31 shows additional linear transport measurements taken after annealing at two different ends of device A. Quantum dots QD2 and QD3, indicated in 
Figure 5.0 are about 2 micron apart. Both Figure 5.3a and Figure 5.3b have the same regularly spaced charge transitions as in Figure 5.26 (right). The charge transitions have equal capacitive coupling to the barrier gates defining the quantum dot and there is no interference from unwanted charge defects. We conclude from Figures 5.2 and 5.3 that our annealing process passivates the majority of defects, and allows formation of hole quantum dots of $180 \mathrm{~nm}$ long.

(a)

(b)
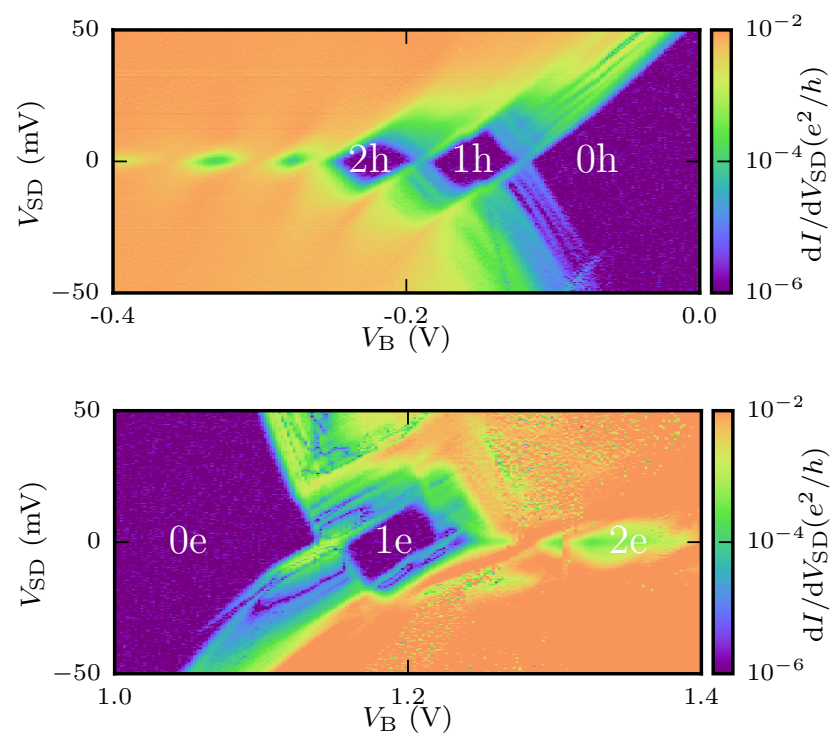

Figure 5.4: Amphoteric nature of the charge center in device B. (a) the hole operation regime $\left(V_{\mathrm{L}}=-1.6 \mathrm{~V}\right)$, and the (b) electron operation regime $\left(V_{\mathrm{L}}=1.7 \mathrm{~V}\right)$ of the same device. Both the hole and electron operation regime show clear charge transitions, with regions of charge stability indicated with $\mathrm{h}$ and e for hole- and electron-occupancy, respectively. The charging energy of the first charge state of both electron and hole are found to be $E_{C} \simeq 10 \mathrm{meV}$.

\subsection{Isolated charge defects}

Now, we will investigate the nature of a single electrically active defect, left after the annealing procedure. Device $\mathrm{B}$, in contrast to device $\mathrm{A}$, contains a layer of $\mathrm{Al}_{2} \mathrm{O}_{3}$ between the $\mathrm{SiO}_{2}$ and the gate electrodes. We have observed that this further increases the yield of our devices. The specific effects of this interlayer on transport characteristics before and after annealing, on the same device, were not studied. After annealing we observe, in all devices with this interlayer, comparable threshold voltages and the same low probability of finding charge traps underneath individual barriers. 
In our ambipolar device structure, the same heterostructure and its associated imperfections can be probed in both the hole and electron operation regime. Figure 5.4 shows a bias spectroscopy of a defect still present after annealing underneath a single barrier. The Coulomb diamonds near the valence band and the conduction band are very similar, and several charge states can be clearly observed. After the last charge transition $(1 \mathrm{~h} \rightarrow 0 \mathrm{~h}$ and $\mathrm{le} \rightarrow 0 \mathrm{e})$ the diamonds opens up and no charge transitions are observed up to a very high bias, for both the hole and electron operation regime. This indicates that the dot has been emptied completely, although without additional verification from e.g. a charge sensing experiment this cannot be said with absolute certainty. The charging energy on both the hole and electron side is found to be $E_{C} \simeq 10 \mathrm{meV}$.

We will now discuss 4 different explanations of these remaining charge transitions, namely dopant atoms, $\mathrm{E}^{\prime}$ centers, unintentional quantum dots, and $\mathrm{P}_{\mathrm{b}}$ centers.

The chance that we are dealing with a dopant atom is small, since our background doping is $\leq 10^{12} \mathrm{~cm}^{-3}$. Additionally, these would not show amphoteric behaviour and charging energies below $20 \mathrm{meV}$.

$\mathrm{E}^{\prime}$ centers, another type of $\mathrm{SiO}_{2}$ defect, are described as non-amphoteric deep hole traps and responsible for fixed positive charge ${ }^{\square-56}$ Primarily induced by radiation, $\mathrm{E}^{\prime}$ centers are often characterized as border traps, ${ }^{\square]}$ located further from the conduction channel. Whether or not this defect could cause Coulomb blockade would depend on its distance from conduction channel. Radiation induced damage caused by electron beam evaporation, ${ }^{44}$ used in our sample fabrication, could lead to higher incidence of this type of defect.

One other explanation for the amphoteric nature of the observed charge transition is the formation of a so-called unintentional quantum dot, created by e.g. disorder or strain. ${ }^{8.3}$ In case of the latter the conduction or valence band is modulated by strain, induced by the difference in thermal expansion coefficients between the silicon and the aluminum gates in the heterostructure. This can create tunnel barriers on each side of the barrier gate, resulting in an unintentional quantum dot. The fact that we observe quantum dot behaviour in a small percentage of barrier gates could be explained by variations in grain size of our constituent $\mathrm{Al}$, resulting in non-uniform strain and tunnel barriers which vary from barrier to barrier.

A system very comparable to the $\mathrm{P}_{\mathrm{b} 1}$ center is the single dangling bond on hydrogen-terminated intrinsic silicon, i.e. without an oxide. Density functional theory (DFT) calculations of this single dangling bond $\left(\mathrm{D}^{0}\right)$ have shown that the associated bound state has an energy level approximately $13 \mathrm{meV}$ above the valence band maximum. ${ }^{54}$ This agrees very well with our extracted value for the charging energy.

The multiple charge states visible in both the hole and electron operation regime preclude us from identifying the simple case of a single $\mathrm{P}_{\mathrm{b}}$ center with only three charge states: $\mathrm{D}^{-}, \mathrm{D}^{0}, \mathrm{D}^{+}$. The origin of the Coulomb diamonds and associated charge states can be explained by the non-singular nature of the defect: Two $\mathrm{P}_{\mathrm{b}}$ 
centers in parallel would also give rise to overlapping Coulomb diamonds, while the different addition energies of the neighbouring Coulomb diamonds would not be expected. It is also known that very proximate dangling bonds can form molecular type bonding, which could account for the observation of more than three charge states. $\underline{54}$

The ability to resolve single $\mathrm{P}_{\mathrm{b}}$ centers could be attributed to the equilibrium density and the corresponding possibility of finding a small but definite number of unpassivated $\mathrm{P}_{\mathrm{b}}$ centers underneath a barrier. This corresponds to our findings that a finite number of barriers still shows an electrically active defect. To confirm this type of model, the device structure proposed in this article combined with statistical analysis on the incidence of charge defects with varying annealing processes would be needed. Electrical detection of spin resonance (EDSR) ${ }^{\amalg 3}$ would further provide strong experimental evidence to confirm if these remaining charge defects are caused by unpassivated $\mathrm{P}_{\mathrm{b}}$ centers.

\subsection{Discussion and conclusion}

In conclusion, we have demonstrated that an annealing process using ALD-grown $\mathrm{Al}_{2} \mathrm{O}_{3}$ is able to passivate the majority of the electrically active defects present at the $\mathrm{Si} / \mathrm{SiO}_{2}$ interface. We attribute this to the passivating properties of hydrogen present in the $\mathrm{Al}_{2} \mathrm{O}_{3}$ layer. After annealing the ability to form electrostatically defined quantum dots markedly improves with dot lengths of at least $180 \mathrm{~nm}$. These quantum dots show many charge transitions, indicating the low level of disorder in the devices. We believe this is most likely due to the elimination of the majority of $\mathrm{P}_{\mathrm{b}}$ centers. The ambipolar device architecture then allows us to reveal the amphoteric behaviour of a remaining charge defect underneath a single barrier with energy levels $\simeq 10 \mathrm{meV}$ above the valence band and below the conduction band.

The ability to form singular quantum dots in this heterostructure is strongly dependent on the density of charge defects at the interface. The control of the spatial density of charge defects in Si planar quantum dots is thus necessary in order to successfully and reproducibly scale up to many devices in a proposed quantum computer. For $\mathrm{P}_{\mathrm{b}}$ centers it is known that the passivation/depassivation reaction leads to an equilibrium, which implies there will always be a finite defect density at the $\mathrm{Si} / \mathrm{SiO}_{2}$ interface. In order to eliminate defects at this interface, another crystalline material to supplant the amorphous $\mathrm{SiO}_{2}$ would be ideal. Candidates for this could either be crystalline dielectrics or other crystalline materials such as SiGe.

The architecture presented here successfully avoids dewetting of $\mathrm{Al}$ in the annealing step by use of $\mathrm{Al}_{2} \mathrm{O}_{3}$, which simultaneously provides hydrogen for the passivation reaction. The ability of the ambipolar architecture to investigate the amphoteric nature of defects could be a very useful tool in the arsenal to further investigate charge defects in all manner of materials. 



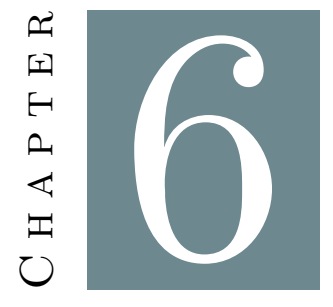

\section{g-tensor anisotropy in a planar silicon hole quantum dot}

The eventual success of quantum computing depends on the ability to isolate and manipulate single spins with great precision. The manipulation of single spins in particular has been achieved in a variety of schemes. Spins can be manipulated by the exchange interaction in exchange-only qubits. ${ }^{157}$ SiGe systems have demonstrated spin manipulation by moving electrons along the electric-field gradient of a proximate micromagnet. ${ }^{158}$ Another option is using microwave pulses resonant with a level transition. The latter case is often technologically achieved by a current-carrying microwave stripline. ${ }^{23}$

A technologically less challenging scheme involves oscillating electric fields to control spins. This is possible in g-tensor modulation resonance, ${ }^{159}$ or electron dipole spin resonance (EDSR). In EDSR the spin and orbit are no longer separable and thus manipulating the dipole element of the electron directly influences its spin. This has been achieved in e.g. InAs $\frac{1411}{14}$ or InSb nanowires, in GaAs heterostructures ${ }^{\square .38}$ where the spin-orbit interaction is intrinsically large. Silicon, being a low-mass element, has a small spin-orbit coupling for electrons. However, the wave-function character of holes in the valence band is primarily p-type, and thus $l=1$, leading to a finite spin-orbit interaction. EDSR has been succesfully demonstrated recently for hole spins in a CMOS compatible Si qubit. Furthermore, hole spins bound to acceptors in Si are another promising system to allow electrical manipulation of spins. ${ }^{\text {16n }}$

A thorough understanding of the spin-orbit mechanisms for holes in Si quantum 
wells is thus necessary. The g-tensor, depending on the spin-orbit interactions, contains information on the wavefunction of its ground state. The shape of the wavefunction can lead to a large anisotropy in the g-tensor, as has been demonstrated in several systems. ${ }^{[28,162}$ For 2 D-hole systems the orientation of hole-spins in a quantum well is expected to be more rigid for $\mathrm{HH}$ than for $\mathrm{LH}$, with $\mathrm{HH}$ states oriented out-of-plane to the 2DHG (see ए2. field out-of-plane of the $2 \mathrm{DHG}$ is expected to cause a greater Zeeman splitting. The g-tensor anisotropy of hole spins dictates how and if electrical noise results in the dephasing of a spin-qubit. In the case of anisotropy a dephasing could be reduced by picking sweet spots, i.e. by choosing the orientation of the magnetic field that results in the longest spin lifetimes.

In this chapter we will demonstrate g-tensor anisotropy of a single hole in a Si planar quantum dot. We use a single-layer electrode design, and remove dangling bonds and charge-defects with annealing, utilizing the hydrogen present in ALD-grown $\mathrm{Al}_{2} \mathrm{O}_{3}$. First we will form a quantum dot in this device, and observe signatures of reaching the single-hole regime. We will continue characterization with ground- and excited-state magnetospectroscopy. Finally, we will follow the (orbital) excited states in a vector magnetic field and extract an effective g-tensor anisotropy.

\subsection{Device design}

(a)

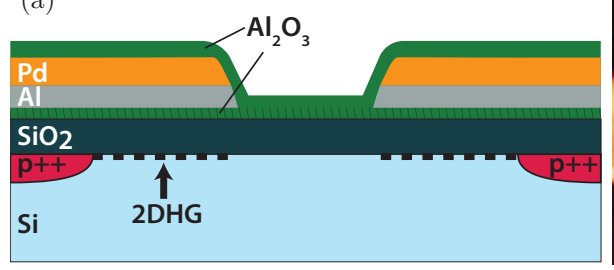

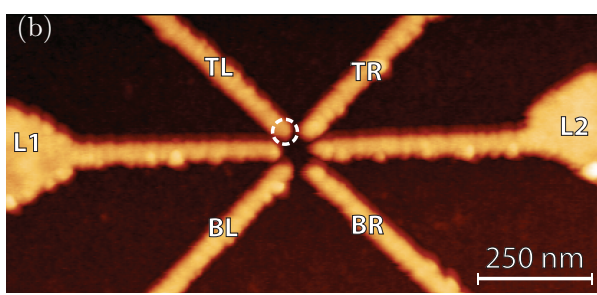

Figure 6.1: Device overview. (a) Schematic cross-section of the device heterostructure, showing the electrodes defined in $\mathrm{Al}(10 \mathrm{~nm})$ and $\mathrm{Pd}(26 \mathrm{~nm})$. (b) Atomicforce micrograph of the device, showing two lead-gates L1 and L2 and the active device area, defined by the top-left (TL), top-right (TR), bottom-left (BL), and bottom-right (BR) electrodes. The approximate location of the dot is indicated by the circle.

The oxidation of the intrinsic silicon and subsequent implantation by-and-large follows the recipe as layed forth by Angus et.al. ${ }^{[\mathrm{LU}}$ After the creation of the highquality oxide window a $5 \mathrm{~nm}$ layer of $\mathrm{Al}_{2} \mathrm{O}_{3}$ is grown at $250{ }^{\circ} \mathrm{C}$. The gate-design is then defined in PMMA using electron-beam lithography (EBL) at an acceleration voltage of $20 \mathrm{keV}$. After development of the PMMA, E-beam evaporation is 
used to deposit the electrode material. First, $\mathrm{Al}(10 \mathrm{~nm})$ is evaporated, followed immediately by $\mathrm{Pd}(26 \mathrm{~nm})$.

After electrode evaporation, $5 \mathrm{~nm}$ of $\mathrm{Al}_{2} \mathrm{O}_{3}$ is grown by $\mathrm{ALD}$ at $100^{\circ} \mathrm{C}$, and the devices subsequently annealed at $400{ }^{\circ} \mathrm{C}$ in $\mathrm{H}_{2}$ at $10 \mathrm{mbar}$ partial pressure for 30 minutes. As a final step, the devices were processed in a UV-ozone reactor for 2 minutes at room temperature. The resulting heterostructure can be seen in Figure 6.1a.

A realized device with single-layer gate design can be seen in Figure 6.76 . The two lead electrodes (L1, L2) coming from the left and right overlap the implanted regions and are used to form a 2DHG that extends to the center of the highquality oxide. Here, the four other electrodes control the potential in the central device area and are designated top-left (TL), top-right (TR), bottom-left (BL), and bottom-right (BR).

\subsection{Initial characterization and dot formation}

Now will characterize the behaviour of such a device at $T=10 \mathrm{mK}$. To form a quantum dot in this design, the following procedure is employed. It is observed that current can flow in parallel current paths, passing either below the bottomhalf electrodes (BL, BR), or below the top-half (TL, TR). By applying a sufficiently high voltage on the bottom-half electrodes the current path underneath it is blocked. Now, the current path can be fully controlled by either of the top-half electrodes. In all measurements presented here, the bottom-part of the device is "turned off". That is, the parallel current path through the bottom is suppressed by tuning $V_{B L}, V_{B R}$ to be well in the region where no current is observed.

In the next step we will operate the device and perform transport measurements as a function of voltages on the top-half of the electrodes, $V_{T L}, V_{T R}$. Figure $\mathbf{6}$. shows the current $I$ through the device as a function of $V_{T L}, V_{T R}$. Several diagonal current lines are irregular and interrupted. We attribute these lines to the charge transitions of an ill-defined quantum dot that is forming somewhere between TL and TR. The vertical lines of increased current $\left(0.8 \mathrm{~V}<V_{T L}<1.3 \mathrm{~V}\right.$, $0.6 \mathrm{~V}<V_{T R}<1.1 \mathrm{~V}$ ) are very strongly coupled to only $\mathrm{TL}$ and is thus most likely located underneath the electrode itself. A bias spectroscopy of this feature is shown in Figure 6.2b. Going from negative to more positive voltages, several Coulomb diamonds with a charging energy $E_{c} \approx 2$ to $3 \mathrm{meV}$ are observed before the last charge transition is seen. The last Coulomb diamond has a charging energy of $E_{c} \approx 10 \mathrm{meV}$. After the last charge transition, the diamond edges open up to $V_{S D}= \pm 20 \mathrm{mV}$. This is an indication that the last hole has been removed from the dot and the single-hole regime has been reached, although this cannot be said with absolute certainty without additional vertification through e.g. a charge-sensing experiment. A faint feature is seen at $V_{T L}=1300 \mathrm{mV}$. The capacitive coupling of this feature to TL is approximately 3 times weaker than for the quantum dot 
underneath TL, and most likely caused by the ill-defined dot forming between TL and TR.

A lockin-technique was used for the rest of the measurements. After optimization, an excitation voltage of $V_{a c}=20 \mu \mathrm{V}$ and frequency $f_{a c}=72 \mathrm{~Hz}$ was chosen. For the last charge transition in Fig. $\mathbf{6 . 2 b}$ we extract lever-arms to the gate TL $\alpha_{g}=0.16$ and to the source $\alpha_{s}=0.58$.

\subsection{Fitting procedures}

The upcoming analysis of the g-tensor anisotropy data requires careful fitting. Therefore we employ two classes of fitting procedures. In both fitting procedures each level, when crossing a bias window, is represented by a Gaussian, given by $f\left(x, A, \sigma, x_{0}\right)$; where A the amplitude, $\sigma$ the standard deviation, and $x_{0}$ the center of the Gaussian.

The first model reconstructs the conductance behaviour as a function of all known parameters across the whole domain/measurement. Taking magnetospectroscopy as an example, in which the conductance is given as $G\left(B_{z}, V_{S D}\right)$, a level can be represented with a clear relation across the domain, such as a simple linear model: $G\left(B_{z}, V_{S D}\right)=f\left(V_{S D}, A, \mu, 0.2 * B_{z}\right)$. This procedure has the advantage that for ill-defined data, the model gives a clear prediction where a level should reside, and the parameters for the Gaussian levels can be singly defined but fitted across the whole domain. An example of this procedure is given in Appendix B.2. In the case of overlapping Gaussians this can be advantageous. We will refer to this procedure as the parametric procedure.

The second model - the simple Gaussian model - fits $n$ separate Gaussians, to a single linetrace (typically as a function of $V_{S D}$ ), and extracts the center $x_{0}$ for each Gaussian to represent the position of the level. This is done for all linetraces and the position of the levels extracted for each parameter. An example of such a fit is given in Appendix $\mathbb{B} . \mathbb{1}$.

Uncertainties reported in this work, for both types of fit, are 2 times the standard error as given by the non-linear least-squares estimator. This represents the $95 \%$ confidence interval.

\subsection{Magnetospectroscopy}

Next we perform ground- and excited-state magnetospectroscopy on the lastvisible transition.

Figure 6.3a shows in-plane magnetospectroscopy of the conductance along the transition. To eliminate the effect of drift of the transition over time, the data has been offset-corrected per linescan of $V_{T L}$. The offset per linescan was determined by cross correlating each linescan of the last-visible transition and by subsequently 
(a)

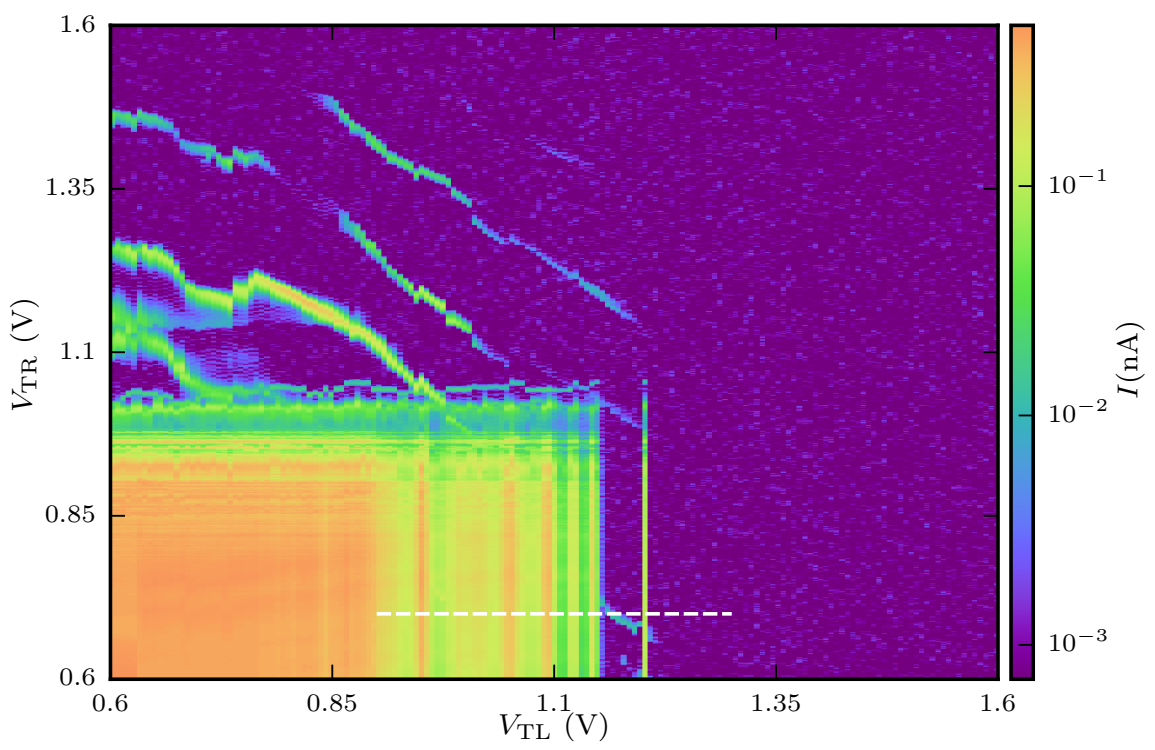

(b)

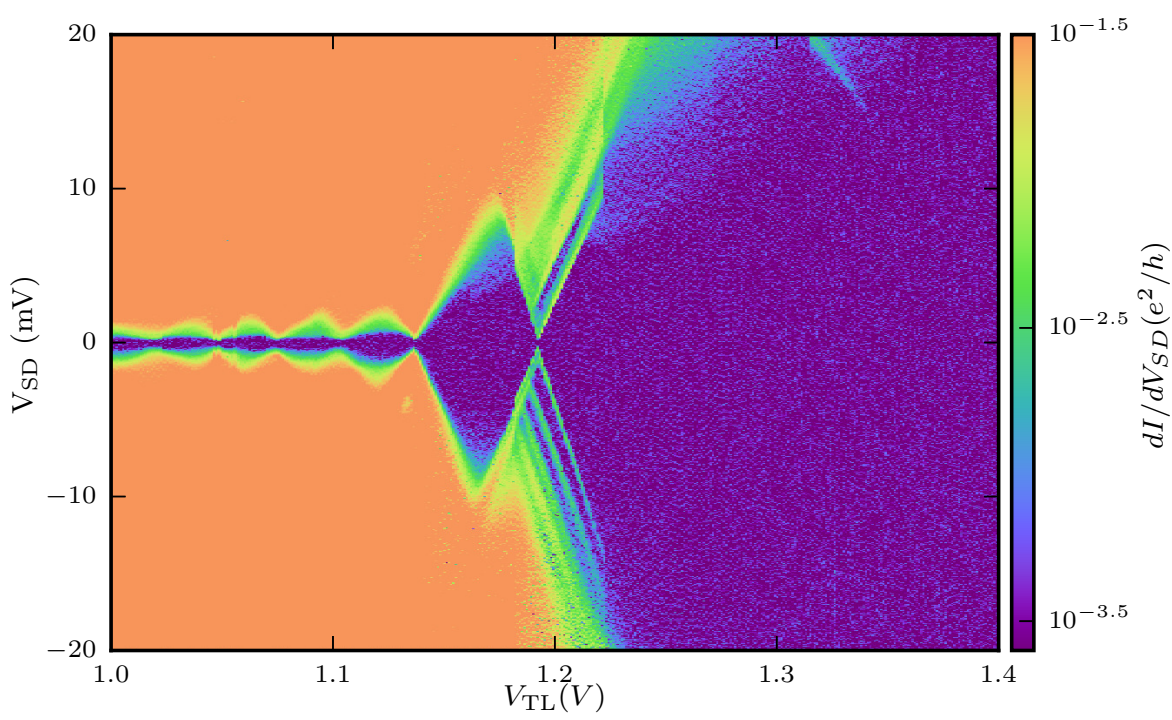

Figure 6.2: Transport characterization of the device at $T \approx 10 \mathrm{mK}$. (a) Linear transport measurement of current $I$ as a function of electrode voltages $V_{T R}$ and $V_{T L}$. Here, $V_{B L}=V_{B R}=1300 \mathrm{mV}, V_{S D}=1 \mathrm{mV}$, and $V_{L 1}=V_{L 2}=-500 \mathrm{mV}$. (b) The differential conductance $d I / d V_{S D}$ as a function of $V_{S D}$ and $V_{T L}$ at $V_{T R}=740 \mathrm{mV}$ (along the dashed line indicated in (a)). 
performing a Gaussian fit on the correlation signal to obtain sub-sample accuracy. This procedure aligns the transition to be nearly stable as a function of magnetic field.

Fitting the corrected data with the parametric procedure for the conductance, which assumes that the transitions move linearly in magnetic field, yields the results seen in Fig. 6.3a. The dashed lines indicate the center of the Gaussian peaks as determined by the fitting procedure. After correction for the gate lever$\operatorname{arm}\left(\alpha_{g}=0.16\right)$, the effective g-factor $g_{z}^{*}$ is calculated (through $\left.E_{z}=g^{*} \mu_{B} B\right)$ to be $1.6 \pm 0.7$.

Excited state spectroscopy of the last-visible transition is shown in Fig. 6.36. The ground-state is seen to split symmetrically with magnetic field. A possible orbital-excited state is visible and also moves in magnetic field parallel to the spinexcited state of the orbital ground-state, starting at $V_{S D} \approx 1.8 \mathrm{meV}$ at $B=0 \mathrm{~T}$. A feature resembling an avoided crossing is indicated by the dashed circle $B= \pm 5 \mathrm{~T}$. This would indicate that, as we will see later, there is an additional level, likely originating from $V_{S D} \approx 1.2 \mathrm{meV}$ at $B=0 \mathrm{~T}$, that crosses the spin-excited state of the orbital ground-state.

A fit with the parametric procedure for the ground state splitting yields an effective g-factor of $g_{z}^{*}=2.21 \pm 0.03$. This is in line with an estimation of the g-factor by eye which results in $g_{z}^{*}=2.2 \pm 0.3$ for the the ground-state. In the same manner, the effective g-factor of the (orbital) excited is estimated to be $g_{z}^{*}=2.5 \pm 0.6$. This assumes that the uncertainty in the lever-arm is negligible compared to the estimation of the slopes.

The uncertainty in the value obtained by fitting is smaller than the evaluation by eye. This could be because the fit does not take into account the possible avoided crossing. Furthermore, the error reported by the fit is misleading as an accurate estimator of the error in the fit, since the standard error is always reduced when more datapoints are used in the fit.

The differing values extracted for the effective g-factor from ground-state spectroscopy (Fig. 6.3a) $\left(g_{z}^{*}=1.6 \pm 0.1\right)$ and the excited-state spectroscopy (Fig. 6.3b) $\left(g_{z}^{*}=2.21 \pm 0.03\right)$ of the last transition could indicate that the last transition $\left(V_{T L} \approx 1183 \mathrm{mV}\right)$ and the previous transition $\left(V_{T L}=1127 \mathrm{mV}\right)$ do not necessarily belong to the same feature. However, the capacitive coupling of the respective transitions indicates that these transitions do belong to the same dot. It could be that the g-factor changes from transition to transition, possibly due to changes in dot-shape which holes are very sensitive to. ${ }^{32}$ This would not influence the excitedstate spectroscopy as shown in Fig. 6.36 as the number of holes on the dot is not changed, in contrast to the measurement in Fig. 6.3a.

Now we will investigate the Zeeman splitting of the ground-state of the lastvisible transition in the three principal magnetic field directions: $\mathrm{x}, \mathrm{y}$, and $\mathrm{z}$. This will allow us to observe the first signs of anisotropy.

A bias spectroscopy at zero magnetic field and for all 3 principal directions at $|B|=1 \mathrm{~T}$ is shown in Figure 6.4. The conductance of the edge of the Coulomb dia- 

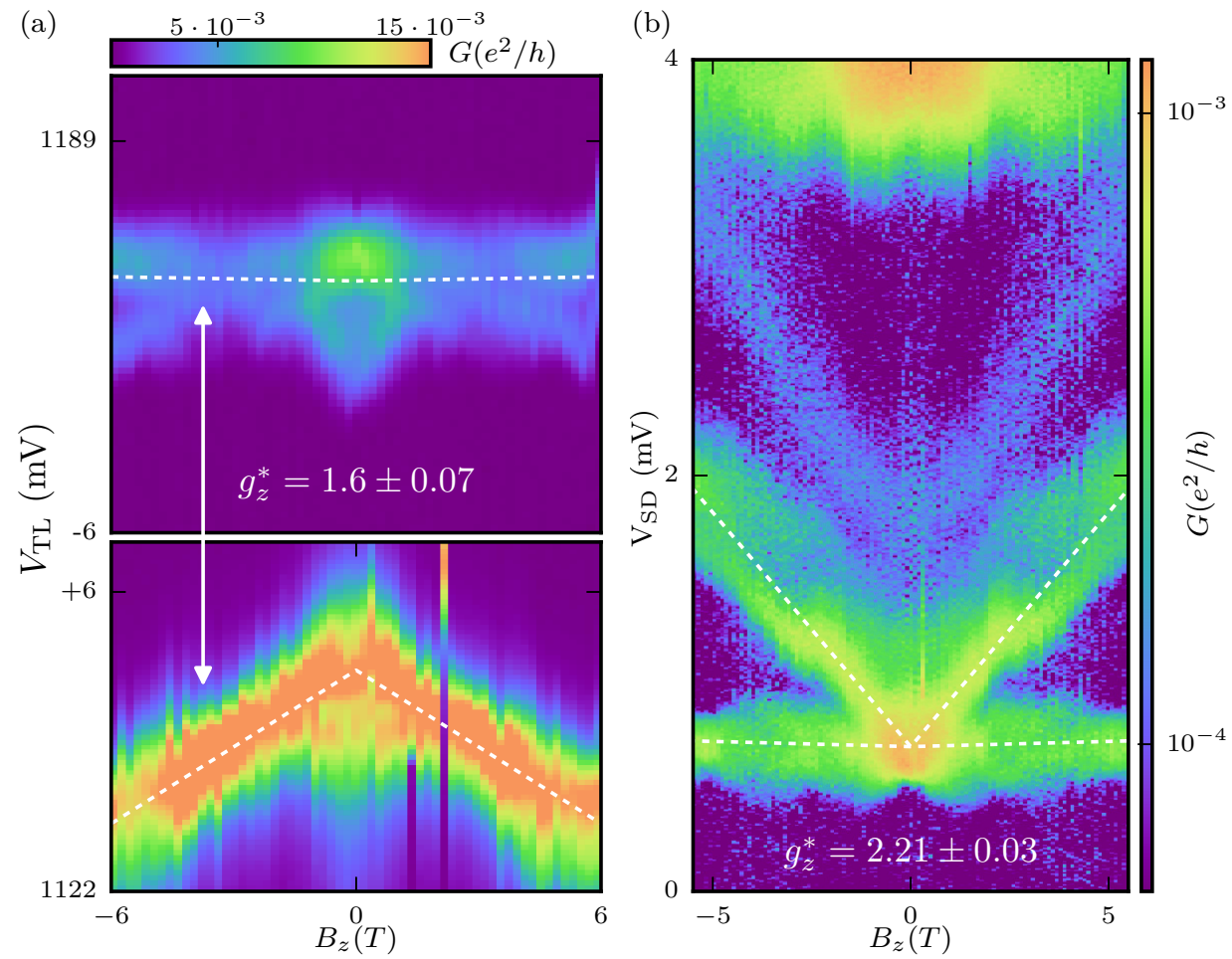

Figure 6.3: Magnetospectroscopy. (a) Ground-state magnetospectroscopy of the first two transitions as a function of voltage difference $\Delta V_{T L}$ to the values $V_{T L}=$ $1122 \mathrm{mV}$ and $V_{T L}=1183 \mathrm{mV}$ for the last and second-to-last transition, respectively. All other gate voltages are the same as in Figure $5 \cdot 2$ and $V_{T R}=740 \mathrm{mV}$. (b) Excited-state magnetospectroscopy of the first transition. The energies have been offset-corrected to the lowest-energy state. The dashed lines are the positions of the center of the Gaussian fits, based on the parametric procedure. $V_{T L}=1186.4 \mathrm{mV}$ 
monds is also seen to decrease when applying $|B|=1 \mathrm{~T}$ for all principal directions, consistent with Zeeman splitting of the ground-state. It can be seen by eye that the splitting of the ground-state is more pronounced when the magnetic field is oriented out-of-plane (along the y-axis). The spin-excited state lines (indicated by the arrows) in positive bias direction reappear in the negative bias direction at the same energies. This is a strong indication that we are dealing with the spin-excited states, and not with extrinsic dot features.

An analysis of the Zeeman splitting across the entire range of $V_{T L}=1182 \mathrm{meV}$ to $V_{T L}=1192 \mathrm{meV}$ of the ground state is fitted with the simple Gaussian approach using 4 Gaussians. The ground-state splitting is calculated to be $\Delta E=$ $(0.27 \pm 0.03) \mathrm{mV}=(0.15 \pm 0.02) \mathrm{meV}$ in-plane, for the $\mathrm{x}$-direction. This results in $g_{x}^{*} \approx 2.7 . \Delta E=(0.27 \pm 0.03) \mathrm{mV}=(0.16 \pm 0.01) \mathrm{meV}$ in-plane, for the z-direction, $g_{z}^{*} \approx 2.7$. These values are a bit higher than the values extracted in Fig. 6.3b. For the out-of-plane, y-direction, the ground-state splitting is calculated to be $\Delta E=(0.35 \pm 0.02) \mathrm{mV}=(0.20 \pm 0.01) \mathrm{meV}$, leading to a g-factor of $g_{y}^{*} \approx 3.5$.

\section{5 g-tensor anisotropy}

Now, we will attempt to measure g-tensor anisotropy by observing the Zeeman energy using a vector magnet and rotating the magnetic field in $3 \mathrm{D}$ in the principal planes at a constant magnetic field of $|B|=1 \mathrm{~T}$. We will then relate the measurements to a simple model.

\subsubsection{The model}

This simple model is based on the Zeeman splitting as dictated by the magnetic field's orientation with respect to the g-tensor. In our coordinate system the gtensor components are defined as follows: $g_{11}=g_{x}, g_{22}=g_{z}$, and $g_{33}=g_{y}=g_{\perp}$. Note here that the out-of-plane direction is the y-axis.

When rotating from in-plane to out-of-plane we will assume that the g-tensor is approximately equal for all components in-plane to the 2DHG i.e. $g_{x, z}=g_{\|}$, and that $g_{33}=g_{y}=g_{\perp}$. We will further assume that all off-diagonal elements for the g-tensor are zero.

For this simple case, the Zeeman energy is given by:

$$
E_{z}=\mu_{B}\left|\left(\begin{array}{ccc}
g_{x}^{*} & 0 & 0 \\
0 & g_{z}^{*} & 0 \\
0 & 0 & g_{y}^{*}
\end{array}\right) \cdot \vec{B}\right|
$$

where $g^{*}$ is the effective g-factor which includes the angular momentum $m_{j}$.

Figure $6.6 \mathrm{a}$ shows the orientation of our sample (red, oriented in the zx-plane) in the magnetic field and the associated angles. The out-of-plane direction is the $\mathrm{y}$-axis, while the $\mathrm{z}$ and $\mathrm{x}$-axis span the plane parallel to the $2 \mathrm{DHG}$. 


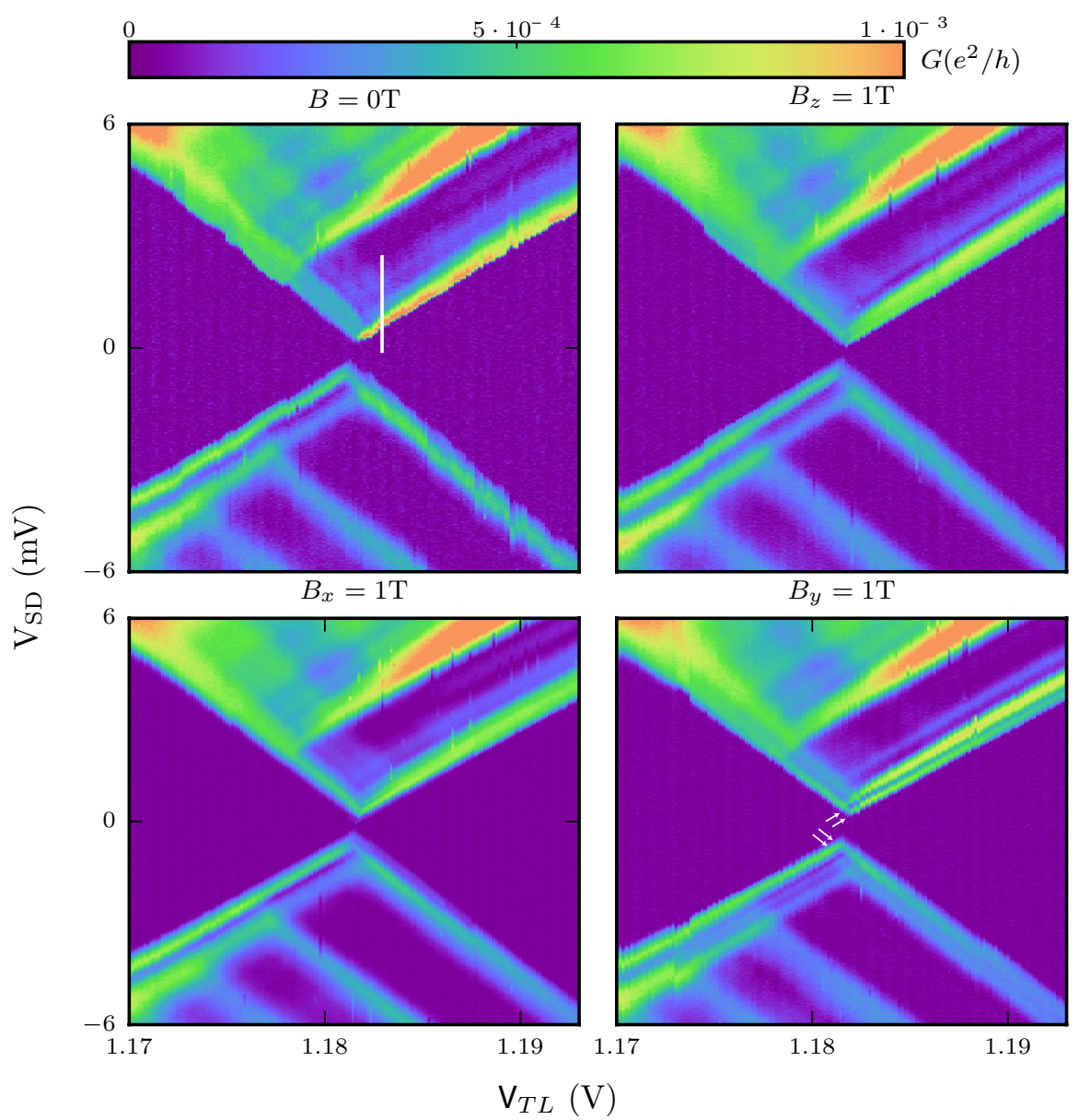

Figure 6.4: Bias spectroscopy of the last visible transition at zero field, and $|B|=1 \mathrm{~T}$ in the three principal directions $\mathrm{x}, \mathrm{y}$, and $\mathrm{z}$. The arrows indicate the spin-split excited states of the ground-state, which are most visible when the magnetic field is oriented out-of-plane along the y-axis. The line in the topleft panel $(\mathrm{B}=0 \mathrm{~T})$ indicates the linecut made in subsequent anisotropy measurements. 
An intuitive picture on what to expect when rotating the B-field around the principal axes can be seen in Figure $\mathbf{6 . 6 6}$. Here, the simple anisotropic model for the Zeeman energy is plotted as a function of the angle $\phi$ for several values of $\theta$, for arbitrary values of $g_{\perp}, g_{\|}$. A maximum Zeeman splitting is achieved for a maximum out-of-plane component of the B-field. Rotating the magnetic field in-plane to the sample yields no dependence on $\phi$.

(a)

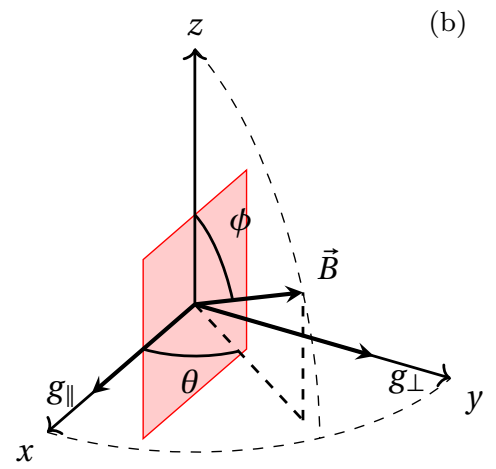

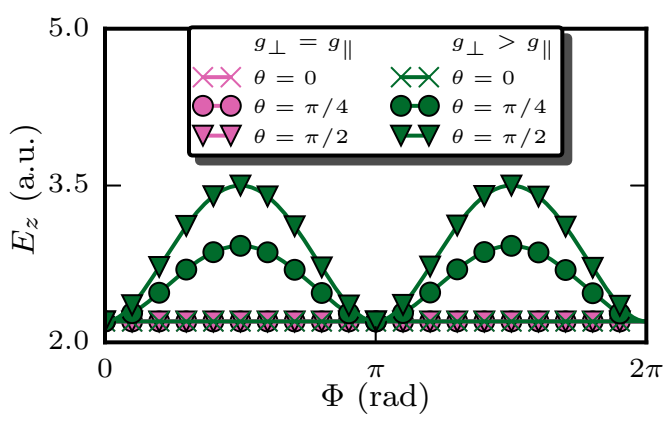

Figure 6.5: 3d g-factor anisotropy (a) The coordinate system used, with the sample oriented in the zx-plane (shown in red). (b) The dependence of the Zeeman energy $E_{z}$ in this coordinate system, for fixed values of $\theta$ and either an isotropic g-factor $\left(g_{\perp}=g_{\|}=2.2\right)$ or according to the simple model of anisotropy $\left(g_{\perp}>g_{\|}\right)$

\subsubsection{Anisotropy on the high-symmetry planes}

As observed earlier, the conductance as a function of $V_{S D}$ is best described by four Gaussians, as can be seen in the fit using the simple Gaussian approach in Fig. B.]. From here on out, we assume that these four levels are present at all times. We will fit the data using both the parametric and simple Gaussian procedure.

We will now observe Zeeman splitting as a function of magnetic field along the principal planes of the magnetic field.

Figs. $\mathbf{6 . 6 0}$, $\mathbf{6 . 6 8}$ and $6.6 \mathrm{~h}$ show the the conductance as a function of $V_{S D}$, and $\phi$ and $\theta$ defining the zy, xy and zx-planes, respectively. The traces were taken at $V_{T L}=1183 \mathrm{meV}$, indicated by the line in Fig. 6.4. The data was offset-corrected to eliminate drift and anomalous moving of the ground-state in energy. This is achieved by offset correction by means of a threshold conductance. The lowest energy state is thus approximately fixed at an arbitrary energy, but only the relative energy differences matter in determining Zeeman energy. This again allows for a clearer indication of ground-state splitting and is easier for fitting. The green and red dots indicate the locations of the levels, as fitted using the simple Gaussian procedure. The pair of dots lowest in $V_{S D}$ (and thus energy) can be attributed to the spin-ground (green) and spin-excited (red) states of the orbital ground- 
state (OGS). The pair of dots highest in $V_{S D}$ can be attributed to the spin-ground (green) and spin-excited (red) states of the orbital excited-state (OES). The OGS has a higher conductance than the OES, and move with changing magnetic field direction.

Rotating the magnetic field from in-plane to out-of-plane in the zy-plane (from the $\mathrm{z}$ to the $\mathrm{y}$-axis), a maximum splitting of the ground-state is reached when the magnetic field is oriented out-of-plane along the y-axis for $\phi=\pi / 2,3 / 2 \pi$ (Fig. [.66). The same can be seen when rotating the magnetic field from in-plane to out-ofplane in the xy-plane where the splitting is minimum for $\theta=0, \pi$ and maximum for $\theta=\pi / 2,3 / 2 \pi$ (Fig. $6.6 \theta)$.

In contrast, when the magnetic field is rotated in the zx-plane, which is in-plane to the 2DHG of the quantum well, barely any movement of the ground-state is seen (Fig. 6.6h). The simple Gaussian procedure reproduces the observation by eye that rotating the magnetic field in-plane has almost no effect on the distance between the spin-excited and spin-ground state of the OGS. There is however a small dependence on B-field orientation. The extracted g-factors in-plane are $g_{x}^{*}=2.55 \pm 0.06$ and $g_{z}^{*}=2.73 \pm 0.06$. The behaviour for moving out-of-plane to inplane is strikingly different, with extracted out of plane g-factors $g_{y}^{*}=3.91 \pm 0.16$ (zy-plane) and $g_{y}^{*}=3.90 \pm 0.10$ (yx-plane). Here, the in-plane g-factor was fixed, to the value previously extracted, at $g_{z, x}^{*}=2.2$. In this model there is also a free parameter $\theta_{0}$ or $\phi_{0}$ which accounts for any rotation of the sample. This could however also compensate for any anisotropy that is intrinsic to the sample. These values are reported in Table $\mathbf{6 . 0}$.

Next, a fit on the same data was done according to the parametric procedure (Fig. 6.7), with the energy levels moving according to the model introduced in Eq. (G.]). The state with the lowest energy is assumed constant (consistent with the thresholding procedure performed on the data), and the spin-excited state is then at a distance $E_{z}$ from the spin ground-state. The dashed lines represent the reconstructed levels as fitted by the model. The fit reproduces the data, with a maximum Zeeman splitting occurring when the B-field is oriented outof-plane and returning to the finite in-plane Zeeman splitting when oriented inplane. The free parameters for the model are the out-of-plane g-factor $g_{\perp}^{*}$, an angle compensating for misalignment in the magnetic field $\theta_{0}$, and parameters pertaining to the Gaussian lineshape of the transition. The in-plane g-factor is kept fixed at the previously assumed value for the in-plane g-factor $g_{\|}^{*}=2.2$. The extracted effective $g$-factors for rotating in the zy and yx plane are $g_{y}^{*}=4.22 \pm 0.02$ (rotated in zy-plane) and $g_{\perp}^{*}(y x)=4.31 \pm 0.02$ (rotated in yx-plane), respectively.

\subsection{3 g-tensor mapping}

In order to fully map the Zeeman splitting and the g-tensor, the conductance was measured as a function of magnetic field by rotating the vector magnet on half of a sphere of radius $\mathrm{B}=1 \mathrm{~T}$, i.e. by changing $\theta=0$ to $\theta \approx 0.43 \pi$ (due to 
(a)

$$
\theta=\pi / 2
$$

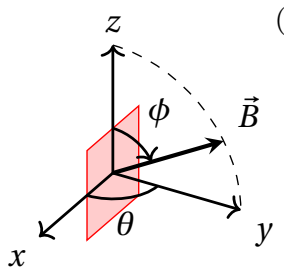

(b) 3.0

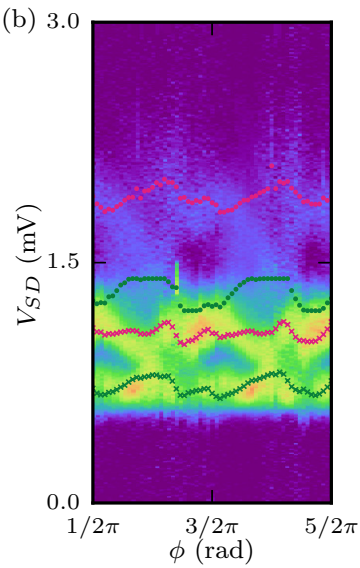

(c)

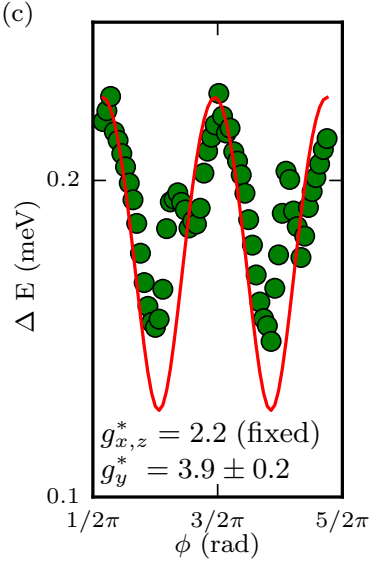

(d)

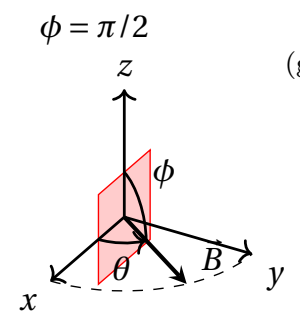

(e)

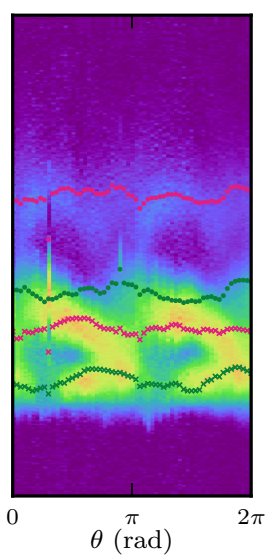

(f)

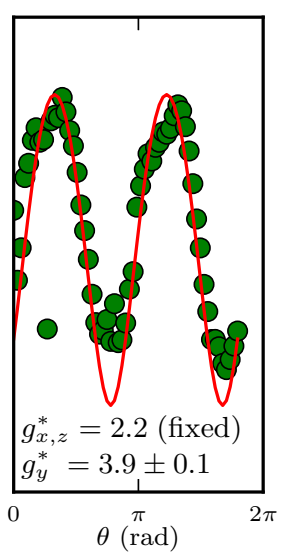

$(\mathrm{g})$

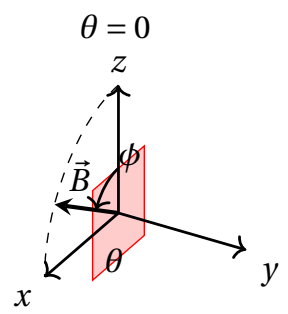

(h)

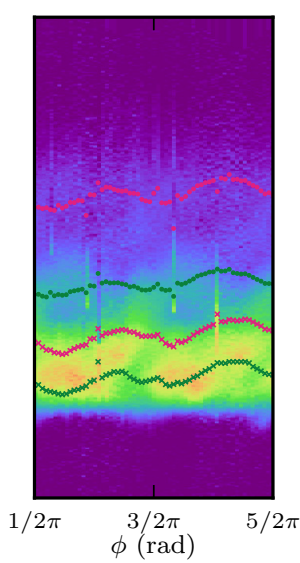

(i)

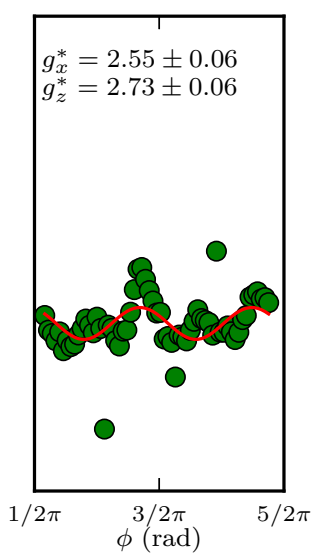

Figure 6.6: Magnetospectroscopy of the last visible transition with magnetic field $|B|=1$ T rotating over the 3 axes of highest symmetry. The positions of the fitted Gaussian centers are indicated by crosses for (b) the zy-plane, (e) the yx-plane, and (h) the zx-plane. (c), (f), (i)] show the respective fit for the Zeeman energy $\Delta E_{z}$ for each magnetic field rotation in a principal plane. 
(a)

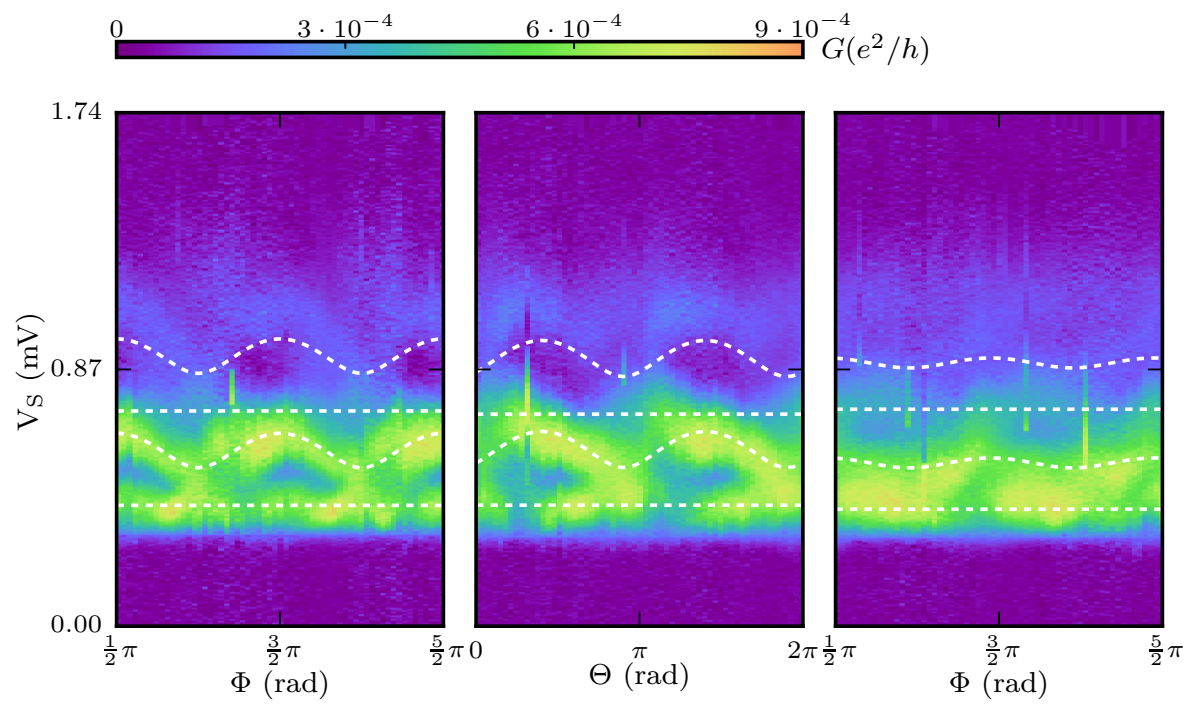

(b)

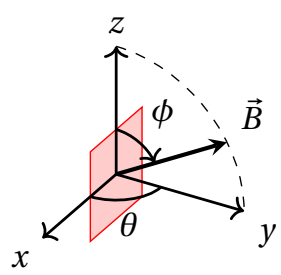

(c)

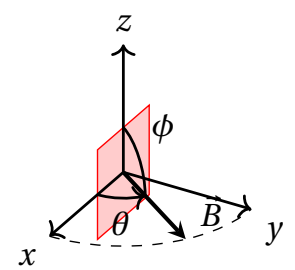

(d)

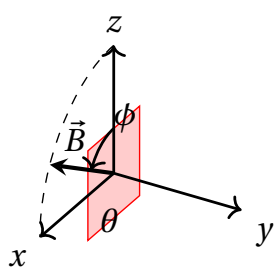

Figure 6.7: Magnetospectroscopy of the last visible transition over the 3 axes of highest symmetry. (b) zy-plane, (c) yx-plane (d) zx-plane. The dashed lines indicate the centers of the Gaussian peaks according to the g-tensor anisotropy model. The model includes a parameter for estimating the misalignment of the sample with respect to the $\mathrm{x}$-y-axis, the angle theta in the model. The in-plane g-factor is fixed to the value extracted in Figure 6.3]. Extracted g-factors are $g_{y}^{*}=4.22 \pm 0.02$ (rotated in zy-plane), $g_{y}^{*}=4.31 \pm 0.02$ (rotated in yx-plane) 
technical difficulties) ${ }^{\square}$, while varying $\phi$ from 0 to $2 \pi$. The added advantage of such a measurement is that any misalignment of the sample on the principal magnetic axes can be reliably accounted for. Fig. 6.7 shows an overview of the entire dataset with the repeated scans in $\phi$ depicted on the x-axis, with $\theta$ changed for each scan in $\phi$. Several zooms of this measurement are given in Fig. 6.7 (c-d) for $\theta=0,0.2 \pi, 0.4 \pi$.

We now fit conductance over this half-sphere. The free parameters in the model are kept the same as previously, with the effective in-plane g-factor fixed at $g_{x, z}^{*}=$ 2.2. Using the parameteric procedure we extract an effective out-of-plane g-factor $g_{y}^{*}=4.74 \pm 0.01$.

A full mapping of the extracted Zeeman level splitting for both the ground- and (orbital) excited-state using the simple Gaussian procedure is given in Fig. 6.9. Here, no g-factors are extracted, but a clear signal is seen indicating that the g-tensor is bigger in the out-of-plane direction. Further anisotropy can also be observed as a ring of increased Zeeman splitting around the out-of-plane direction at approximately $45^{\circ}$ to the y-axis. This could be an artifact of the fitting procedure, or could be be caused by alignment of the magnetic field with e.g. the [111] crystal direction.

Table 6.1: All extracted values for the effective g-factor with relevant parameters for the three different methods: Gaussian fitting, 2D fitting and 2D fitting on the half-sphere. Errors given are the 95\% confidence interval (twice the standard error as reported by the least-square algorithm).

\begin{tabular}{|c|c|c|c|c|c|}
\hline & \multirow[b]{2}{*}{$g_{y}^{*}$} & \multirow[b]{2}{*}{$g_{z}^{*}$} & \multirow[b]{2}{*}{$g_{x}^{*}$} & \multicolumn{2}{|c|}{ Misalignment } \\
\hline & & & & $\theta_{0}(\mathrm{rad})$ & $\phi_{0}(\mathrm{rad})$ \\
\hline simple Gaussian & & & & & \\
\hline zy-plane & $3.91 \pm 0.16$ & $2.2($ fixed) & 2.2(fixed) & - & $0.01 \pm 0.04$ \\
\hline yx-plane & $3.90 \pm 0.10$ & 2.2(fixed) & 2.2(fixed) & $0.42 \pm 0.06$ & - \\
\hline zx-plane & - & $2.73 \pm 0.06$ & $2.55 \pm 0.06$ & - & $0.5 \pm 0.2$ \\
\hline parametric & & & & & \\
\hline zy-plane & $4.22 \pm 0.02$ & 2.2(fixed) & 2.2 (fixed) & - & - \\
\hline yx-plane & $4.31 \pm 0.02$ & 2.2(fixed) & 2.2(fixed) & $0.306 \pm 0.006$ & \\
\hline zx-plane & - & $2.419 \pm 0.016$ & $3.002 \pm 0.012$ & - & $0.16 \pm 0.02$ \\
\hline parametric full & $4.74 \pm 0.01$ & $2.2($ fixed $)$ & $2.2($ fixed $)$ & 0.5 (fixed) & - \\
\hline
\end{tabular}

\subsection{Discussion and conclusion}

We have succesfully created and characterized a hole quantum dot and measured its g-tensor in a single-layer device in intrinsic silicon. A single-layer device is technologically attractive since it has a reduced complexity in fabrication, which

\footnotetext{
${ }^{1}$ This is of course scientist vernacular for when things go entirely pear-shaped - in contrast to our g-tensor which is most likely cigar-shaped.
} 
(a)

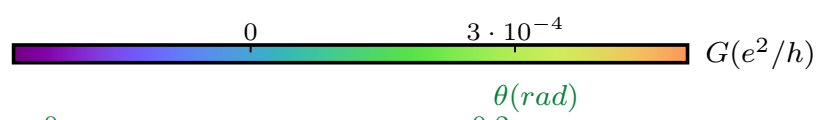

$\theta(\mathrm{rad})$

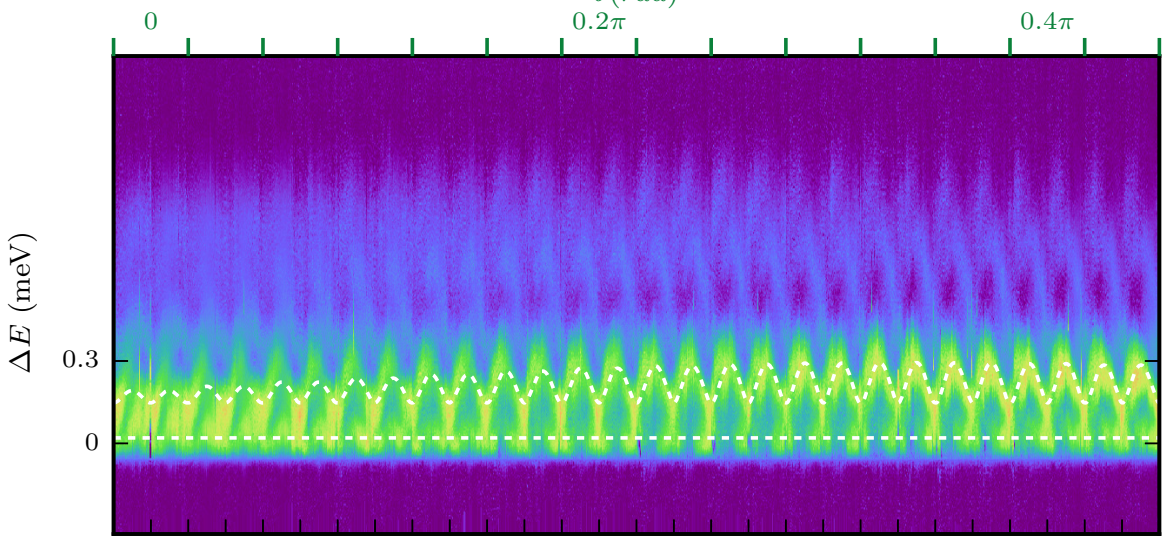

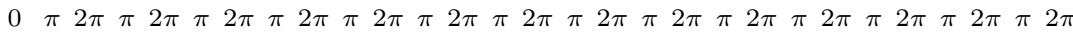
$\Phi(\mathrm{rad})$

(b)

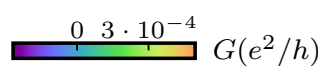

$\theta=0$

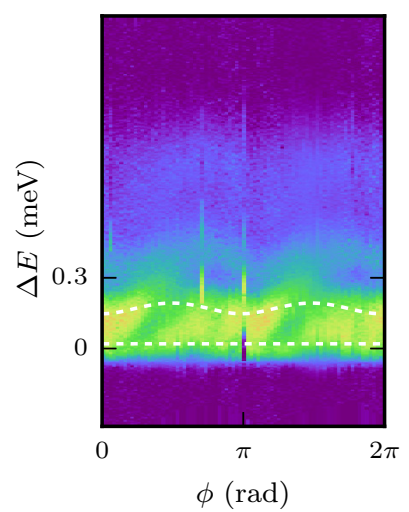

(c)

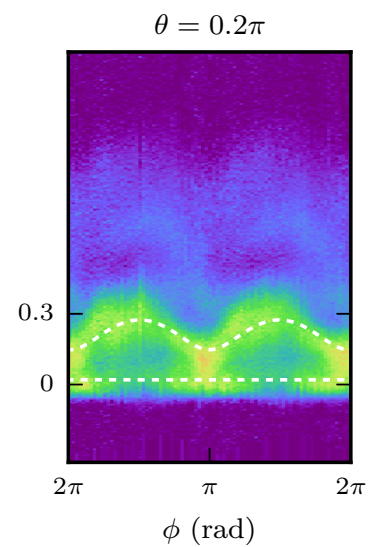

(d)

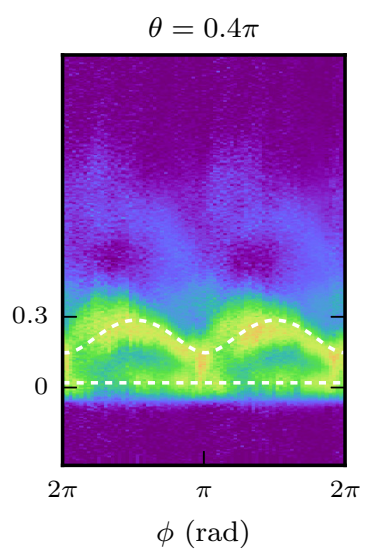

Figure 6.8: g-tensor mapping over the half-sphere (a) Conductance as a function of the offset-corrected energy $\Delta E$ for and $\phi$ and all $\theta$ from $\theta=0$ to $\theta=\pi / 2$. The dashed line is the extracted center of the Gaussian in a fit of the model. The extracted g-factors are $g_{y}^{*}=4.74$ and $g_{z, x}^{*}=2.2$ (fixed) (b-d) shows the zooms of the plots at $\theta=0, \theta=\pi / 4$, and $\theta=\pi / 2$ 
(a)

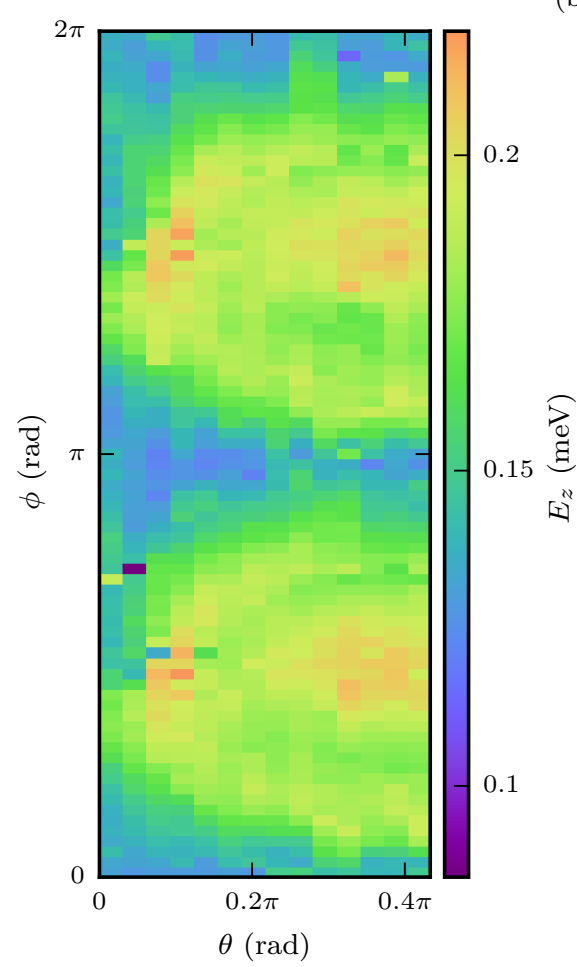

(b)

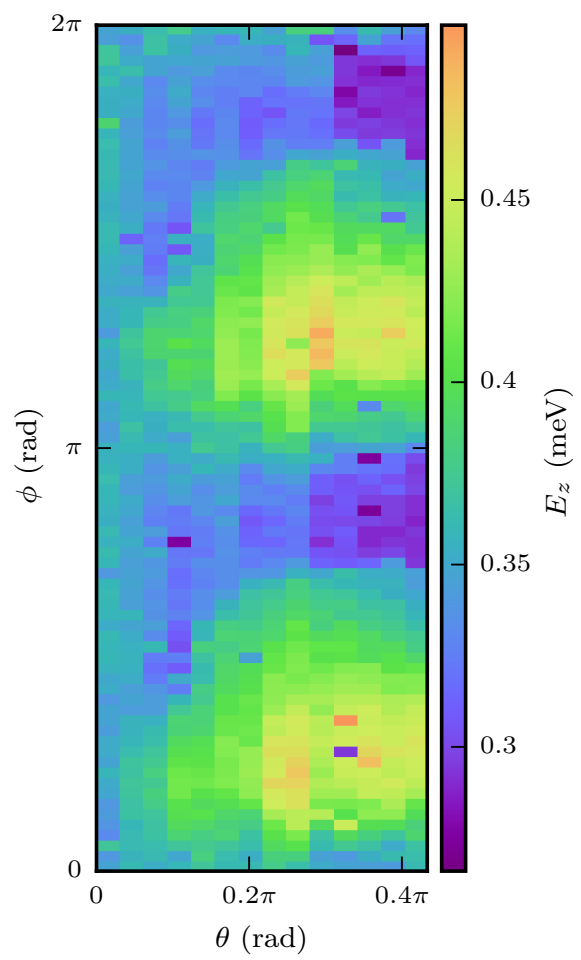

Figure 6.9: g-tensor mapping over the half-sphere using the simple Gaussian procedure (a) The Zeeman energy as extracted for the ground state as a function of vector magnet angle $\theta$ and $\phi$. (b) Same as (a) but for the extracted Zeeman energy of the possible (orbital) excited state.

leads to an increased yield, and reduces any damage which accumulates with each processing step. The quantum dot formed in this device could be a manifestation of several effects. A depletion-type operation might come about due to the existence of fixed charge, which is known to form in ALD-grown $\mathrm{Al}_{2} \mathrm{O}_{3}$ after annealing. $\frac{119,163}{163}$ it is also known that thermally induced strain can cause the formation of unintentional quantum dots, ${ }^{\times 3}$ which the use of $\mathrm{Al}$ as a gate electrode here would exacerbate due to its high thermal expansion coefficient. Another option is that the quantum dot is a $\mathrm{P}_{\mathrm{b}}$ center or other type of charge defect, which was not passivated in the annealing process.

The finite Coulomb gap at the last transition could be an indication that there is a parasitic dot in series or parallel which is preventing transport for low sourcedrain bias. This could be the irregular dot that was observed to form between TL and TR. 
We have shown the behaviour of the ground and (orbital) excited state in a vector magnetic field. We observe no four-fold $(\mathrm{HH} / \mathrm{LH})$ degeneracy of the groundstate as observed previously on an acceptor in $\mathrm{Si} . \stackrel{150}{\square}$

It is known that holes are more sensitive to g-tensor modulation by changing of dot shape, and possibly also electric fields. ${ }^{[2,164}$ This could account for the observed difference in g-factor of the ground state for the last-visible and the following transition, as the shape and size of the dot are not known.

Employing a Zeeman-splitting model based on g-tensor anisotropy we extract a g-factor that is approximately two times higher out-of-plane than it is in-plane. We do this for the principal planes (xy, yx, zx), as well as by mapping the conductance over roughly half the sphere that the vector magnet can cover at $|B|=1 \mathrm{~T}$. The values extracted in all measurements and fitting procedures $\left(g_{\|}^{*} \approx 2.2, g_{\perp}^{*} \approx 4\right)$ show the same trend: a roughly two-fold larger g-factor out-of-plane than in-plane $\left(g_{\perp}^{*} / g_{\|}^{*} \approx 1.8\right)$. This is consistent with the prediction that LH and HHs are oriented preferentially in a $2 \mathrm{D}$ quantum well. ${ }^{\text {W }}$ A full g-tensor map over half of $3 \mathrm{D}$ space shows additional detail for the Zeeman splitting of the OGS in the form of a symmetrical ring. This could indicate additional texture of the g-tensor, but is more likely an artifact of the method of fitting.

Other possible reasons for the observed anisotropy could be a reduction in the tunneling probability for the states, which can be seen to diminish as well for certain magnetic field directions. However, solid tracking of the levels using the simple Gaussian procedure seems to be contrary to this assumption.

The general trend in the data presented here that the g-tensor is anisotropic bodes well for the future of holes as quantum bits. The anisotropy could allow for spin manipulation through EDSR, g-tensor modulation, and allow tuning of the magnetic-field orientation to pick sweet spots to optimize coherence. 



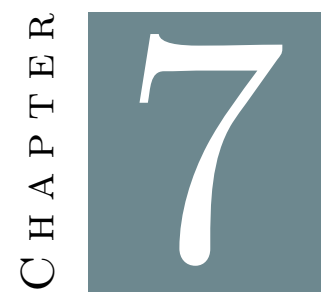

\section{Single-layer depletion-mode hole quantum dots in intrinsic silicon}

We have seen in Chapter [3, that the negative fixed charge present in ALD-grown $\mathrm{Al}_{2} \mathrm{O}_{3}$ after annealing can induce a $2 \mathrm{D}$ hole gas (2DHG) without an applied gate voltage.

This 2DHG, combined with only a single layer of gate electrodes, are all the ingredients necessary to create a depletion-mode type quantum dot, similar to devices created in GaAs/AlGaAs or doped Si/SiGe heterostructures. This chapter will serve as a proof-of-principle on the creation of such devices, and features a gate design that is not optimized yet for dot shape or single-hole occupation.

\subsection{Confinement}

There are several ways to define quantum dots (QDs) in the solid-state. One is the vertically oriented quantum dot, first defined in GaAs nanopillars. ${ }^{\text {[3 }}$ The second is the lateral confinement depletion-mode quantum dot, in which the few-electron limit was reached some years later. ${ }^{\sqrt{65}, \| 66}$ The last is the enhancement mode design, originally motivated by improving the ease with which the few-electron regime can be reached.

Compared to electrons, holes in Si have more stringent confinement requirements, due to their effective mass being higher or at least comparable to those of electrons. Typical values cited for the effective masses are the transverse electron 
mass $m_{t}^{*}=0.19 m_{0}$, and $m_{h h}^{*}=0.53 m_{0}$ for heavy holes, the expected ground state in quantum dot wells. Since the lateral dimensions as defined in enhancement mode devices directly dictate where charge accumulates, this links the dimensions of the dot strongly to the lithographic dimensions. Depletion-mode designs employ a more open design, in which the field lines spread out further than the lithographic dimensions, which could allow for better confinement. The ability to more accurately control dot shape could be used to optimize for maximum sensitivity to spin-manipulation.

A depletion-mode quantum dot relies on the presence of a conducting state when no voltages are applied to electrodes. The already present $2 \mathrm{DHG}$ is locally depleted by applying positive voltages to the gate electrodes to form the tunnel

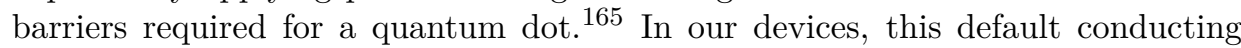
state is provided by the electric field of negative fixed charge in ALD-grown $\mathrm{Al}_{2} \mathrm{O}_{3}$ that has been annealed at $400^{\circ} \mathrm{C}$.

Another aspect of our device is that $\mathrm{Pd}$ is used as the metal for the gate electrodes. TEM studies have shown that the use of $\mathrm{Pd}$ as a gate electrode material does not form an oxide interlayer that is typically present in planar Si quantum dot architectures based on $\mathrm{Al}$ electrodes (see Fig. B.100). To assess the improvement of $\mathrm{Pd}$ on $\mathrm{Al}$, we turn to charge-offset stability as a metric. Charge offset is the variation of background charges in a quantum dot device. In quantum dot heterostructures this is often caused by glassy media such as oxides with two-level fluctuators (TLFs). ${ }^{[34}$ Stability of background charge is very important in quantum dots, since it can shift the electrochemical potential of the quantum dot over time, resulting in unwanted drift. It was shown that metal-based single-electron devices show charge-offset stabilities typically on the order of $0.5 e$, and Al-based on the order of $0.15 e$.

One of the ways to extract the charge offset is by taking the trace of a number of Coulomb oscillations as a function of gate voltage $V_{g}$. The oscillations are modelled as a function of regularly interspaced peaks and fitted using the following general formula: $\frac{\pi 0}{}$

$$
I_{D}\left(V_{g}\right)=A_{0}+A \sin \left(2 \pi V_{g} / \Delta V_{g}+Q_{0}(t) / e\right)+B V_{g}
$$

with $A_{0}$ the base current, $A$ the amplitude of the peaks, $B$ accounts for a linear increase of background current. The charge offset is given as $Q_{0}=\frac{\text { phase }}{\Delta V_{g}} e$, with $\Delta V_{g}$ the periodicity of the Coulomb oscillations. In this manner charge offset is expressed as a shift of the Coulomb oscillations, expressed in the number of (fractional) charge transitions the dot undergoes. The charge-offset stability is expressed as the range over which the charge-offset drifts over the measurement time period.

Usings these two improvements, we show a simple single-layer depletion-mode design that, while still unoptimized, shows single- and double-dot behaviour and a charge-offset stability of $<0.05 e$ measured over a period of 82 hours at a temperature of $T \approx 10 \mathrm{mK}$. 


\subsection{Gate design}

(a)

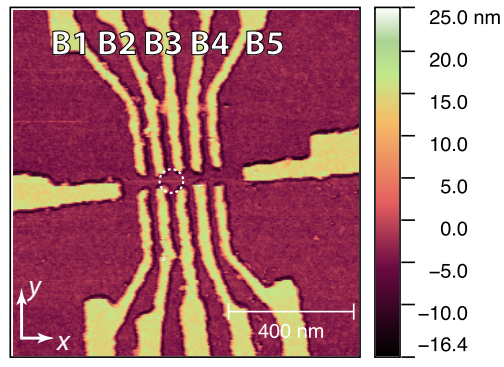

(b)

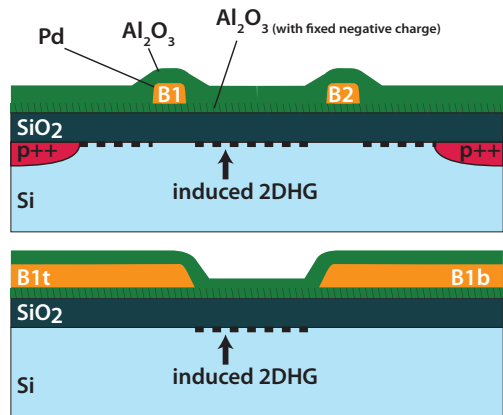

Figure 7.1: Depletion quantum dot design (a) AFM image of a device (b) (top panel) cross-sectional view (as seen looking along the $x$-axis of (a)) with the 2DHG remaining between the electrodes that have depleted the 2DHG underneath them. (bottom panel) cross-sectional view (as seen looking along the $y$-axis of (a) where the conduction path is visible.

The gate design consists of 10 electrodes that come in from the top and bottom (see Fig. [. ald). Electrodes opposite each other have the same voltages applied to them, leading to the designation B1 through B5 for all pairs of gates. If a different voltage is applied to either top or bottom electrode, we will append a " $\mathrm{t}$ " or " $\mathrm{b}$ " designating top or bottom, respectively.

The electrodes at the far-left (L) and far-right (R) (Fig. R.Ja) are present in the design for extra tunability but are not used.

\subsection{Tuning between a single and double quantum-dot}

In the particular device under study, B4 and B5 were not showing any effects on conduction in the channel, therefore we focus on the 3 left-most electrode pairs $\mathrm{B} 1, \mathrm{~B} 2$, and B3.

We will now try to form a quantum dot between B1 and B3, and use B2 as a plunger. The method is as a follows: the voltage on B1 and B3 is tuned to sufficiently high voltages that the $2 \mathrm{DHG}$ underneath them is depleted and conduction in the channel is nearly suppressed. The expectation is that, due to the gate geometry, the gap between the top and bottom electrodes remains sufficiently conducting as to act as the tunnel barrier.

Conduction through the channel could be turned off by applying sufficient voltages on a pair of gates to deplete the $2 \mathrm{DHG}$ underneath and between them. It is expected the $\mathrm{Al}_{2} \mathrm{O}_{3}$ dielectric facilitates an easy coupling of the electric field to the gap between the pair of gates, which is why it can be easily depleted. 
Figure $\left[2.2\right.$ shows the current $I$ as a function of $V_{B 1}$ and $V_{B 3}$, at four values of the plunger barrier $V_{B 2}$. By varying $V_{B 2}$ from $0.2 \mathrm{~V}$ to $0.55 \mathrm{~V}$, we see diagonal lines (coupling equally to B1 and B3) changing into lines more capacitively coupled to B1 and B3 separately. This can be interpreted as the initially formed quantum dot gradually splitting in two by introducing an interdot tunnel barrier which we control by the voltage $V_{B 2}$. As we increase $V_{B 2}$, the interdot coupling gradually decreases, and honeycomb-like patterns are observed, indicative of a double quantum dot.

For the region in gatespace where a single quantum dot is formed $\left(V_{B 2}=0.20 \mathrm{~V}\right)$, we observe discontinuities in the charge transitions which extend vertically, and are coupled strongly to B1. This indicates that there is an unintentional quantum dots present, most likely underneath, or in the vicinity of, B1.

\subsection{Optimizing a single quantum dot}

In order to study the charge-offset stability of this device, we will form a single quantum dot between B2 and B3. We start this process by focusing on the charge transitions belonging to the dot beneath B3, indicated by the dashed line crossing the transitions in the top-left panel of Fig. [.2. By tuning B1 to very negative voltages, the dot that is associated with $\mathrm{B} 1$ (the nearly vertical charge transition lines in Fig. [2:), is eliminated.

Fig. [7.3b shows the current $I_{S D}$ as a function of $V_{B 2 t}$ and $V_{B 3 t}$. The charge transitions observed are equally coupled to both B2t and B3t, which indicates that the dot is formed between B2 and B3. We also observe a line crossing the transitions associated with the dot between B2 and B3, shifting its place in gatespace. This could indicate that the dot we have formed is not fully decoupled from a nearby (unintentional) quantum dot or the dot retains a slight double quantum dot character.

Fig. [.3a shows a bias spectroscopy of the dot between B2 and B3. For optimial stability, only the voltage on the top electrode $V_{B 3 t}$ is varied in this measurement. The charging energy of the last-visible transition is measured to be $E_{C} \approx 15 \mathrm{meV}$, and is an indication of reaching the single-hole regime. This is comparable to the charging energy of previously obtained results for the last electron in Si. 64, ,

\subsection{Charge-offset stability}

Having tuned to a region where we have well-defined Coulomb peaks, we now turn to a measurement of the charge-offset stability. This measurement is performed by measuring the current $I_{S D}$ while varying $V_{B 3 t}$ over the charge transitions of the quantum dot between B2 and B3. The gate voltage $V_{B 3 t}$ is varied in both the forward $(600 \mathrm{mV}$ to $1300 \mathrm{mV})$ and backward $(1300 \mathrm{mV}$ to $600 \mathrm{mV})$ direction, while 


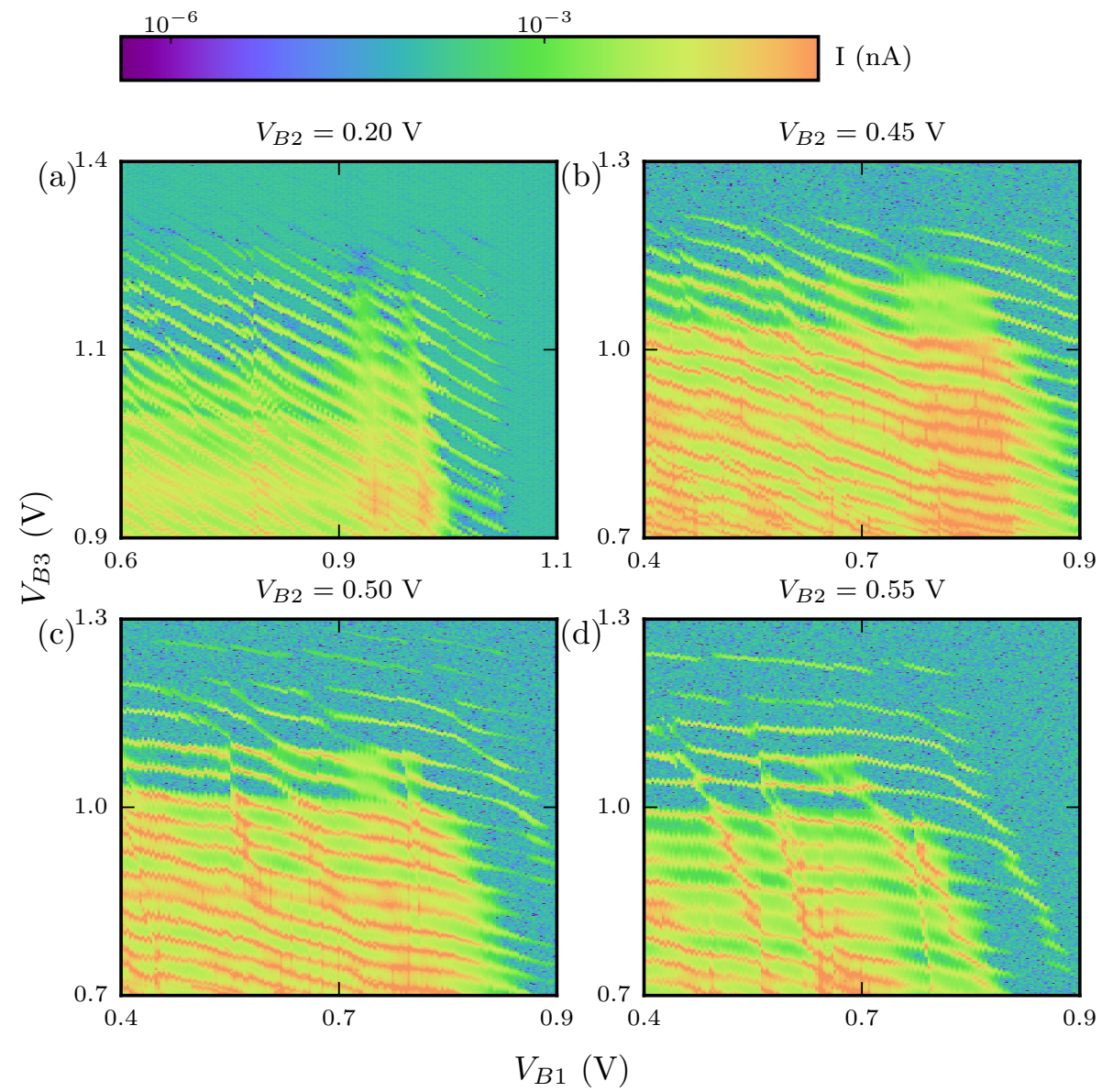

Figure 7.2: Linear transport measurements of current $I_{S D}$ as a function of gate voltage $V_{B 1}$ and $V_{B 3}$. Here, $V_{S D}=1 \mathrm{mV}, V_{B 4}=V_{B 5}=0 \mathrm{mV}$, and $V_{L}=V_{R}=-200 \mathrm{mV}$. As the plunger gate $V_{B 2}$ is varied from $0.20 \mathrm{~V}$ to $0.55 \mathrm{~V}$, the charge transitions change from equally coupled to B1 and B3 (signifying a single quantum dot) to honeycomb-like structures and 2 sets of charge transition lines coupling strongly to B1 and B3 separately (signifying a double quantum dot). 
(a)

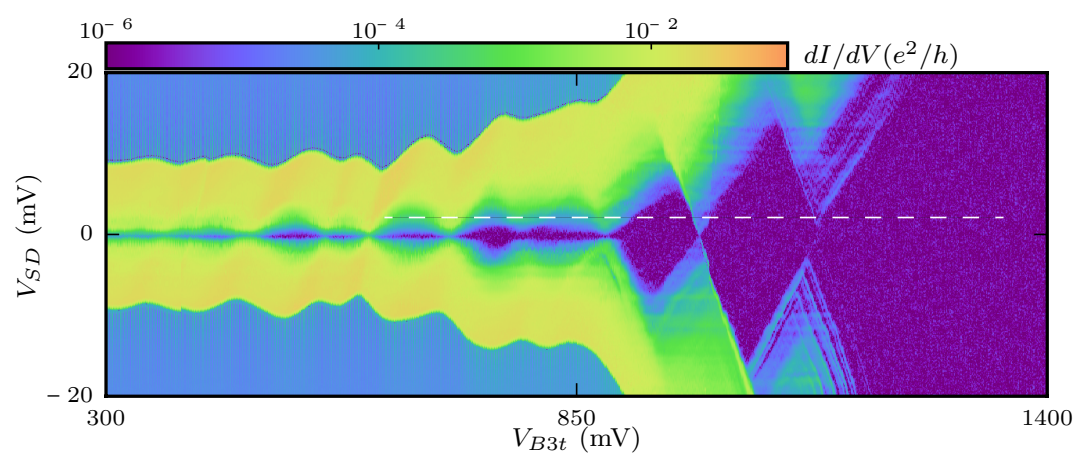

(b)

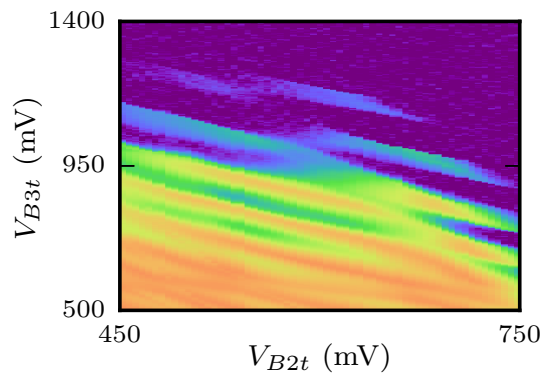

Figure 7.3: Characterization of the quantum dot under consideration for chargeoffset stability. (a) Bias spectroscopy with $V_{B 3 b}=1500 \mathrm{mV}, V_{B 1}=-2000 \mathrm{mV}, V_{B 2}=$ $650 \mathrm{mV}$ (b) Coupling of quantum dot between B2 and B3, where $V_{S D}=-6 \mathrm{mV}$.

the source-drain voltage is set to $V_{S D}=2.5 \mathrm{mV}$ (the dashed line in Fig. [r.3a). This is done continuously over a timespan of 82 hours, resulting in 370 traces of the Coulomb peaks.

To extract the charge-offset stability, instead of fitting a periodic function as in Eq. (ㅁ. $)$, we utilize a cross-correlation method. We analyse each separate transition by taking the first linetrace of $I_{S D}$ as a function of $V_{G}$ and correlating each subsequent linetrace to it. The extracted offset in voltage is simply divided by our Coulomb peak spacing $\Delta V_{g} \approx 139 \mathrm{mV}$. We assume that the lineshape does not change over time. This has the advantage that there is no underlying assumption about the peak-shape, such as is done when utilizing a simple sine-fit as done previously. ${ }^{168}$ We do this separately for all scans taken in the forward and backward direction.

The charge offset for the last two visible charge transitions at $V_{B 3 t} \approx 1152 \mathrm{mV}$ (transition 1) and $V_{B 3 t} \approx 1013 \mathrm{mV}$ (transition 2), are shown in Fig. [.4a. An initial variation of the charge-offset (0-3 hours) followed by a period of bigger stability can be seen. This can be attributed to an initial settling time of the transition after changing the gate voltages to reach the desired transition. A further statistical analysis of this data is shown in the histograms for the last Fig. $7.4 b$ and second- 
to-last transition Fig. 7.4b. We can observe a qualitatively different distribution for the forward and backward sweep direction. The forward direction shows a bimodal distribution of the charge offset, while the backward direction exhibits a decidedly more centered distribution. The bimodal distribution comes about through an apparent bistable pattern in charge offset, that can be observed in the time domain. To extract the distribution of the charge-offset we assume a standard distribution and fit with a Gaussian function. The extracted parameters are given in Table $[$.$] . Transition 1$ has the biggest variation in $Q_{0}$, and from the histogram we can see that $Q_{0}$ varies $0.04 e$ over the course of the measurement. Transition 2 is exceptionally stable in the backward direction, with $Q_{0}$ varying $0.005 e$ over the course of the measurement.

Table 7.1: Extracted distribution of the mean $\mu$ of the charge-offset $Q_{0}$, where \pm indicates the standard deviation $\sigma$ of $Q_{0}$. In case of the bimodal distribution two separate values are given, one for each component in the distribution.

\begin{tabular}{c||l|l}
\multicolumn{1}{c||}{} & \multicolumn{2}{c}{$\left[\mu_{Q_{0}} \pm \sigma_{Q_{0}}\right](e / 100)$} \\
\hline transition 1 & & \\
forward & $0.67 \pm 0.23$ & $2.0 \pm 0.27$ \\
backward & $1.2 \pm 0.2$ & \\
\hline transition 2 & & \\
forward & $0.1 \pm 0.2$ & $-1.4 \pm 0.3$ \\
backward & $0.18 \pm 0.16$ & \\
\hline
\end{tabular}

\subsection{Discussion and conclusion}

We have shown transport behaviour of a single-layer depletion-mode hole quantum dot. Transport measurements indicate that the device can form both single and double quantum dots, by varying the voltage on an interdot coupling barrier. By further optimization we have obtained a quantum dot that shows signs of the few-hole regime, where the last visible charge-transitions have charging energies of $E_{c} \approx 10 \mathrm{meV}$ and $E_{c} \approx 15 \mathrm{meV}$. The equal capacitive coupling of the quantum dot to B2 and B3 is a strong indication that the dot is in fact created between the electrodes, and is an intentional quantum dot made by operating in depletion-mode. Unintentional quantum dots, such as those caused by defects or temperature-dependent strain are expected to couple very strongly to only one electrode.

The charge-offset stability has an upper bound of $Q_{0}=0.04 e$ and a lower bound of $Q_{0}=0.005 e$. The sweep direction had a noticeable influence on the stability of the background charges. This could have to do with the filling of charge traps nearby or TLF stability during that particular measurement sequence. The results compare favourably to the previous known results for Al-based single-electron devices which show a stability of $Q_{0}<0.15 e$, and are on-par with all-Si based devices which 
(a)

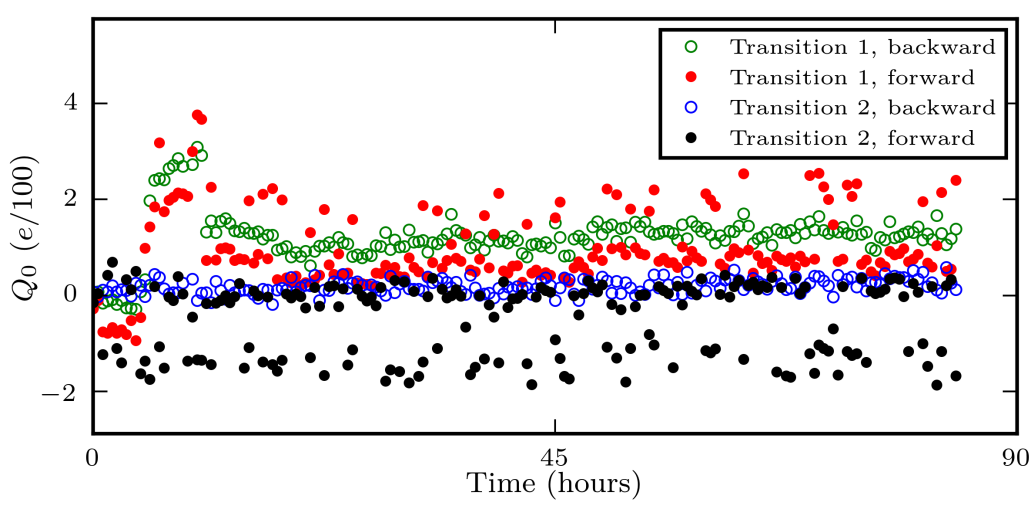

(b)

(c)
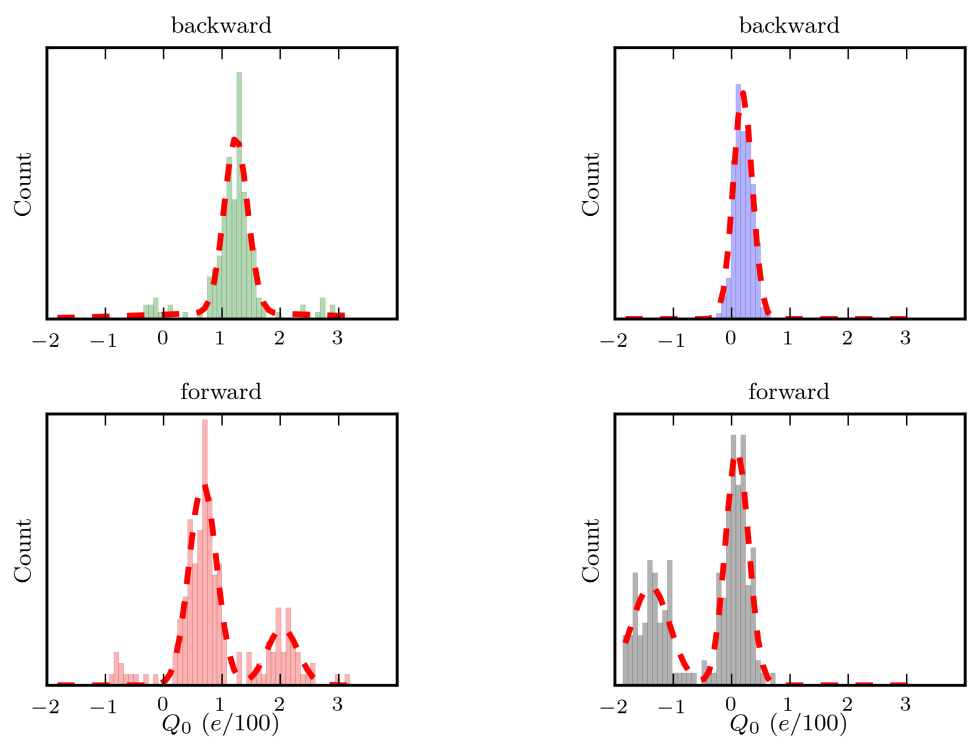

Figure 7.4: Charge-offset stability over 82 hours. (a) Charge offset over time of the two last charge transitions. (b) histogram (60 bins) of the charge offset of transition 1 for both the forward and backward measurement direction. (c) histogram (60 bins) of the charge offset of transition 2 for both the forward and backward measurement direction. 
shows charge stabilities of $Q_{0}<0.01 e$. Both of these values were obtained at $T=2.2 \mathrm{~K}{ }^{\square .78}$ Results on all-Si devices at temperatures near $T=20 \mathrm{mK}$ showed similar results. ${ }^{134}$ Therefore it can be expected that temperature is not the limiting factor in measuring charge-offset stability. For completeness, we mention that the electron temperature of our measurement setup is expected to be $T_{e} \approx 35 \mathrm{mK}, \square[2$ which is lower than the electron temperature of the known reference measurements of $T_{e} \approx 250 \mathrm{mK}$. $\frac{\text { प68 }}{\mathrm{T}}$

These results further corroborate the idea that the interfacial oxide layer, which was not detected for our device in TEM studies of the $\mathrm{Pd} / \mathrm{Al}_{2} \mathrm{O}_{3}$ interface, is largely responsible for long term charge-offset instabilities.

The design and heterostructure, as presented here, make for a very simple system to create depletion-mode quantum dots. The well-defined Coulomb diamonds when a single quantum dot is formed are promising, and optimization can push this design even further. This optimization should take care to absorb the lessons learned in previous designs, tum dots reaching single-hole occupation. A possible additional advantage of a single-layer depletion-mode design is that it avoids damage from any electronbeam lithography in the areas where dots are actively formed. The open nature of the design also means that future experiments could more easily couple external sources of light into the structure. Given these features, the depletion-mode design has its place alongside the enhancement-mode design, and could again prove useful in experiments in Si. 



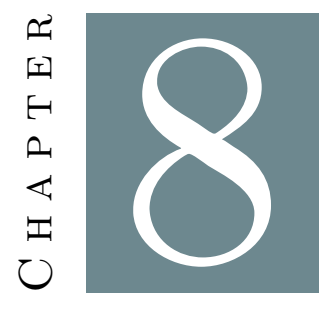

\section{Conclusions and outlook}

In this thesis we have addressed some of the challenges that arise in making hole quantum dots in a planar Si architecture.

\subsection{Hole quantum dots}

We have explored a scalable CMOS compatible architecture capable of single-hole tunneling through hole quantum dots. Without an annealing process, we found that many charge defects are found underneath single barrier electrodes. After developing an annealing process with ALD-grown $\mathrm{Al}_{2} \mathrm{O}_{3}$, we have shown that the majority of these charge defects can be passivated. This enables the creation of clearly defined hole quantum dots, and clears a path to study single holes in silicon.

The annealing process allowed us to determine the anisotropy of the g-tensor, measured by observing Zeeman splitting as function of a directionally varying magnetic field. This behaviour is telling of the rich physics the spin-orbit interaction facilitates in the valence band. Hole-spin dynamics in Si have certainly not been experimentally explored in great detail. The spin-orbit interaction could be exploited for the manipulation of spins on single acceptor atoms, ${ }^{\text {"6] }}$ or be used to manipulate the effective mass as a function of strain. $\underline{\text { Bu }}$

A next step in exploring the spin-orbit coupling can be sought in the confinement potential for holes. For quasi-2D quantum wells, manipulating the confinement potential would alter the g-tensor anisotropy. 1 In the context of quantum computation, this can be exploited in g-tensor modulation resonance. ${ }^{\square-59}$ In our 
architecture, a possible scheme involves introducing a backgate, which is able to change the slope of the potential in the quantum well. This is easily accomplished in a silicon-on-insulator substrate, where a highly-doped substrate can be used as a gate. The influence of lateral confinement, i.e. the shape of the dot, which influences the correlated spin-orbit currents, ${ }^{12}$ can also be investigated by changing the gate layout of our architecture, or by manipulating the voltages on the quantum-dot forming electrodes.

\subsection{Heterostructures}

In creating a structure to form quantum dots in, we cannot escape the influence of the host heterostructure and its many interactions.

We have studied the Si planar quantum dot heterostructure using TEM, and shown that the interfacing a metal and an oxide can lead to formation of interfacial oxide. This oxide layer forms when using $\mathrm{Al}$ and $\mathrm{Al}_{2} \mathrm{O}_{3}$, or $\mathrm{Al}$ and $\mathrm{SiO}_{2}$, but does not form when using a combination of $\mathrm{Al}_{2} \mathrm{O}_{3}$ and $\mathrm{Pd}$. The associated glassy dynamics of oxide that go with it are expected to improve, which should be reflected in charge-offset stability of devices. This is very important when considering reproducibility at scale-up.

A predicted additional effect in heterostructures is also the existence of temperaturedependent strain, resulting in tunnel barriers at opposite ends of metal gate electrodes. ${ }^{83}$ This has cast a new light on the use of Al in Si planar heterostructures. There are two options: exploit the effect, or eliminate it by switching to materials with a smaller expansion coefficient. To eliminate the effect, two candidate materials that have a small expansion coefficient and are technologically most suitable are TiN and poly-Si.

\subsection{1 $\mathrm{P}_{\mathrm{b}}$ centers}

Given the well-known result that hydrogen-annealing passivates $\mathrm{P}_{\mathrm{b}}$ centers, it is very likely that most of the defects seen before our annealing process are $\mathrm{P}_{\mathrm{b}}$ centers. The passivation of $\mathrm{P}_{\mathrm{b}}$ centers using hydrogen is a well known result but not previously studied in great detail for intrinsic silicon, low temperatures, or for individual defects. The architecture used in this work then also affords the study of charge defects in many other systems. The low temperature and ambipolar architecture make possible the detailed study of the local structure of the material and individual defects, in a regime not accessible through other methods.

It is unclear if the remaining few charge defects we have observed are device variations or $\mathrm{P}_{\mathrm{b}}$ centers. Future devices would use poly-Si gates or TiN, in order to exclude strain-related phenomena. If caused by $\mathrm{P}_{\mathrm{b}}$ centers, $\mathrm{P}_{\mathrm{b}}-\mathrm{H}$ complexes, $\mathrm{E}$ ' centers, or heretofore unknown defects, the charging energy can be predicted by theoretical calculations. As these defects are measured in quasi-2D quantum 
wells, with possible additional lateral confinement potentials and electric fields, it is possible the defects behave different from their normal ensemble state in chemical equilibrium. This could be seen as $\mathrm{P}_{\mathrm{b}}$ centers isolated from the rest of their ensemble. In this sense, comparing the $\mathrm{P}_{\mathrm{b}}$ center to isolated dangling bonds on $\mathrm{Si}$ are a good starting point to further the understanding of this defect. $\underline{\text { b7, }, 7.3}$

It is important to realize that $\mathrm{P}_{\mathrm{b}}$ centers cannot be eliminated entirely, due to the passivation kinetics causing a finite equilibrium spatial density of defects. In a scenario where the architecture is scaled up, this can become disastrous, as a fixed percentage of devices will be disturbed by the electrically active defect. Possible improvements could be had in applying a voltage bias during annealing, which would reduce the defect density even further, but still leave us with a finite density.

Further study of this defect will benefit from a correlation of $D_{i t}$ extracted from capacitance-voltage measurements to the charge defects found underneath barriers.

The existence of defects in the $\mathrm{Si} / \mathrm{SiO}_{2}$ architecture is worrying, and the equilibrium densities of $\mathrm{P}_{\mathrm{b}}$ centers after annealing suggest a scaled up version of the architecture would suffer from these. Barring the existence of a phase of $\mathrm{SiO}_{2}$ better suited to the Si, i.e. one that would form no dangling bonds, the best solution would be to resort to a crystalline insulating material. Undoped Si/SiGe heterostructures have already shown much promise and could pave the way of sidestepping the problem associated with these defects altogether. also envision other (crystalline) dielectrics better suited to epitaxially match the Si underneath.

\subsection{Depletion-mode quantum dots}

We have shown that ALD-grown $\mathrm{Al}_{2} \mathrm{O}_{3}$ can prevent dewetting of thin metal films, as well as provide the hydrogen to passivate defects. We have also drawn attention to the fact that this oxide can contain negative fixed charge under certain annealing conditions. The negative fixed charge has the ability to induce hole conductivity in the MOSFET structure through a gating effect. It was also shown that a UVozone treatment can reduce this fixed charge to such a degree that this "induced" conductivity is reduced to $\sigma<10^{-10} \mathrm{~S}$.

The effect of an extra gate that comes essentially "for free" could also be used to our benefit. Given the negative fixed charge, we have created single-layer hole depletion-mode style dots. In this mode of operation, the fixed charge induces a 2DHG across the whole device, and a quantum dot is formed by locally depleting the gas by applying a positive voltage to the electrodes. 


\subsection{Single-electron/single-hole transistors}

The flexibility of gate layout, combined with the ability to conduct both holes and electron in ambipolar quantum dots allows the creation of single hole- and electrontransistors (SET/SHT) in close proximity to one another. One of the quantum dots could be tuned to function as a charge-sensor, while the other quantum dot is being studied. Besides the possibility of charge-sensing this might allow investigation of more fundamental physics in $\mathrm{Si}$, such as phonon-assisted electron-hole recombination.

This could be realized in two ways. First, a depletion/accumulation hybridmode could be imagined. The SHT would be induced by negative fixed charge in ALD-grown $\mathrm{Al}_{2} \mathrm{O}_{3}$ and charge depleted by electrodes, whereas the SET is formed by accumulation of electrons underneath the electrodes. Using UV-ozone, the accumulation-mode operation of both SET and SHT would be recovered.

We can even envision a system where the SHT/SETs are scaled up even further, with a regular grid of closely packed hole and electron quantum dots which, due to the indirect bandgap, would recombine very rarely at cryogenic temperatures. Stimulation of electron-hole recombination in this system, with e.g. surface acoustic waves, would be an interesting extension of this idea.

\subsection{Final thoughts}

The promise of computing using quantum resources has come a long way since its conception in the early 70s. The involvement of more and more industry is a testament to the maturity of the field. One might optimistically estimate that we are halfway there. Luckily, technological progress has a tendency to exponentially increase its speed, so that the final push should not take another 50 odd years. That said, that does not mean there are many more challenges to overcome.

These challenges express themselves among others in quantum dot heterostructures, which remains an arena where material science, fabrication, and fundamental quantum mechanics are in a delicate balance. In this light, the prospects for holes as carriers for spin-information is broad, rich in physics, and very bright. 


\section{References}

1. R. P. Feynman, presented at the annual meeting of the American Physical Society.

2. A. Hagar, M. Cuffaro, in The Stanford Encyclopedia of Philosophy, ed. by E. N. Zalta (Metaphysics Research Lab, Stanford University, Summer 201, 2015).

3. R. P. Feynman, Simulating physics with computers. International journal of theoretical physics 21, 467-488 (1982).

4. D. Deutsch, Quantum Theory, the Church-Turing Principle and the Universal Quantum Computer. Proceedings of the Royal Society A: Mathematical, Physical and Engineering Sciences 400, 97-117 (July 1985).

5. L. K. Grover, Quantum Mechanics Helps in Searching for a Needle in a Haystack. Physical Review Letters 79, 325-328 (July 1997).

6. P. W. Shor, presented at the Foundations of Computer Science, 1994 Proceedings., 35th Annual Symposium on, pp. 124-134.

7. E. Schrödinger, Die gegenwärtige Situation in der Quantenmechanik. Naturwissenschaften 23, 823-828 (1935).

8. T. Ferris, The Whole Shebang (Simon and Schuster, 1998), p. 345.

9. J. I. Cirac, P. Zoller, Quantum computations with cold trapped ions. Physical Review Letters 74, 4091 (1995).

10. D. G. Cory, A. F. Fahmy, T. F. Havel, Proceedings of PhysComp '96. (1996).

11. A. G. Fowler, M. Mariantoni, J. M. Martinis, A. N. Cleland, Surface codes: Towards practical large-scale quantum computation. Physical Review A Atomic, Molecular, and Optical Physics 86 (2012).

12. D. Loss, D. P. DiVincenzo, Quantum computation with quantum dots. Physical Review A 57, 120-126 (Jan. 1998).

13. S. Tarucha, D. Austing, T. Honda, R. van der Hage, L. Kouwenhoven, Shell Filling and Spin Effects in a Few Electron Quantum Dot. Physical Review Letters 77, 3613-3616 (1996). 
14. J. M. Elzerman, R. Hanson, L. H. Willems van Beveren, L. M. K. Vandersypen, L. P. Kouwenhoven, Excited-state spectroscopy on a nearly closed quantum dot via charge detection. Applied Physics Letters 84, 4617-4619 (June 2004).

15. J. R. Petta, A. C. Johnson, J. M. Taylor, E. A. Laird, A. Yacoby, M. D. Lukin, C. M. Marcus, M. P. Hanson, A. C. Gossard, Coherent manipulation of coupled electron spins in semiconductor quantum dots. Science (New York, N.Y.) 309, 2180-4 (Sept. 2005).

16. F. Koppens, C. Buizert, K. Tielrooij, I. Vink, K. Nowack, T. Meunier, L. Kouwenhoven, L. Vandersypen, Driven coherent oscillations of a single electron spin in a quantum dot. Nature 442, 766-771 (2006).

17. M. Steger, K. Saeedi, M. L. W. Thewalt, J. J. L. Morton, H. Riemann, N. V. Abrosimov, P. Becker, H.-. J. Pohl, Quantum Information Storage for over 180 s Using Donor Spins in a ${ }^{28} \mathrm{Si}$ "Semiconductor Vacuum". Science 336 , 1280-1283 (June 2012).

18. J. J. Pla, K. Y. Tan, J. P. Dehollain, W. H. Lim, J. J. L. Morton, F. A. Zwanenburg, D. N. Jamieson, A. S. Dzurak, A. Morello, High-fidelity readout and control of a nuclear spin qubit in silicon. Nature 496, 334-338 (Apr. 2013).

19. S. Goswami, K. A. Slinker, M. Friesen, L. M. McGuire, J. L. Truitt, C. Tahan, L. J. Klein, J. O. Chu, P. M. Mooney, D. W. Van Der Weide, et al., Controllable valley splitting in silicon quantum devices. Nature Physics 3, 41-45 (2007).

20. S. J. Angus, A. J. Ferguson, A. S. Dzurak, R. G. Clark, Gate-Defined Quantum Dots in Intrinsic Silicon. Nano Letters 7, 2051-2055 (July 2007).

21. W. H. Lim, H. Huebl, L. H. Willems van Beveren, S. Rubanov, P. G. Spizzirri, S. J. Angus, R. G. Clark, A. S. Dzurak, Electrostatically defined few-electron double quantum dot in silicon. Applied Physics Letters 94, 173502 (2009).

22. N. S. Lai, W. H. Lim, C. H. Yang, F. A. Zwanenburg, W. A. Coish, F. Qassemi, A. Morello, A. S. Dzurak, Pauli Spin Blockade in a Highly Tunable Silicon Double Quantum Dot. Scientific Reports 1 (2011).

23. M. Veldhorst, J. C. C. Hwang, C. H. Yang, a. W. Leenstra, B. de Ronde, J. P. Dehollain, J. T. Muhonen, F. E. Hudson, K. M. Itoh, A. Morello, a. S. Dzurak, An addressable quantum dot qubit with fault-tolerant controlfidelity. Nature Nanotechnology 9, 981-985 (Oct. 2014).

24. M. Veldhorst, C. H. Yang, J. C. C. Hwang, W. Huang, J. P. Dehollain, J. T. Muhonen, S. Simmons, A. Laucht, F. E. Hudson, K. M. Itoh, A. Morello, A. S. Dzurak, A two-qubit logic gate in silicon. Nature 526, 410-414 (Oct. 2015). 
25. Z. Zhong, Y. Fang, W. Lu, C. M. Lieber, Coherent Single Charge Transport in Molecular-Scale Silicon Nanowires. Nano Letters 5, 1143-1146 (June 2005).

26. F. A. Zwanenburg, C. E. W. M. van Rijmenam, Y. Fang, C. M. Lieber, L. P. Kouwenhoven, Spin States of the First Four Holes in a Silicon Nanowire Quantum Dot. Nano Letters 9, 1071-1079 (Mar. 2009).

27. R. Li, F. E. Hudson, A. S. Dzurak, A. R. Hamilton, Pauli Spin Blockade of Heavy Holes in a Silicon Double Quantum Dot. Nano Letters 15, 7314-7318 (2015).

28. B. Voisin, R. Maurand, S. Barraud, M. Vinet, X. Jehl, M. Sanquer, J. Renard, S. De Franceschi, Electrical control of g-factors in a few-hole silicon nanowire MOSFET. Nano Letters (2015).

29. M. H. Kolodrubetz, J. R. Petta, Coherent Holes in a Semiconductor Quantum Dot. Science 325, 42-43 (2009).

30. T. Guillaume, M. Mouis, Calculations of hole mass in [110]-uniaxially strained silicon for the stress-engineering of p-MOS transistors. Solid-State Electronics 50, 701-708 (Apr. 2006).

31. R. Winkler, D. Culcer, S. J. Papadakis, B. Habib, M. Shayegan, Spin orientation of holes in quantum wells. Semiconductor Science and Technology 23, 114017 (Nov. 2008).

32. J. van Bree, A. Y. Silov, M. L. van Maasakkers, C. E. Pryor, M. E. Flatté, P. M. Koenraad, Anisotropy of electron and hole g tensors of quantum dots: An intuitive picture based on spin-correlated orbital currents. Physical Review B 93, 035311 (2016).

33. A. V. Kuhlmann, J. Houel, A. Ludwig, L. Greuter, D. Reuter, A. D. Wieck, M. Poggio, R. J. Warburton, Charge noise and spin noise in a semiconductor quantum device. Nature Physics 9, 570-575 (2013).

34. N. M. Zimmerman, W. H. Huber, B. Simonds, E. Hourdakis, A. Fujiwara, Y. Ono, Y. Takahashi, H. Inokawa, M. Furlan, M. W. Keller, Why the longterm charge offset drift in Si single-electron tunneling transistors is much smaller (better) than in metal-based ones: Two-level fluctuator stability. Journal of Applied Physics 104 (2008).

35. Y. Nishi, K. Tanaka, A. Ohwada, Study of Silicon-Silicon Dioxide Structure by Electron Spin Resonance II. Japanese Journal of Applied Physics 11, 85-91 (Jan. 1972).

36. P. J. Caplan, E. H. Poindexter, B. E. Deal, R. R. Razouk, ESR centers, interface states, and oxide fixed charge in thermally oxidized silicon wafers. Journal of Applied Physics 50, 5847-5854 (1979).

37. T. Heinzel, Mesoscopic Electronics in Solid State Nanostructures, ed. by T. Heinzel (Wiley-VCH Verlag GmbH, Weinheim, Germany, Nov. 2006), pp. 247-272. 
38. R. Hanson, L. P. Kouwenhoven, J. R. Petta, S. Tarucha, L. M. K. Vandersypen, Spins in few-electron quantum dots. Reviews of Modern Physics 79, 1217-1265 (2007).

39. M. R. A. Shegelski, The chemical potential of an ideal intrinsic semiconductor. American Journal of Physics 72, 676-678 (May 2004).

40. R. Winkler, Spin-Orbit Coupling Effects in Two-Dimensional Electron and Hole Systems (Springer Berlin Heidelberg, Berlin, Heidelberg, 2003), vol. 191.

41. A. Alkauskas, M. D. McCluskey, C. G. Van De Walle, Tutorial: Defects in semiconductors - Combining experiment and theory. Journal of Applied Physics 119 (2016).

42. C. Freysoldt, B. Grabowski, T. Hickel, J. Neugebauer, G. Kresse, A. Janotti, C. G. Van De Walle, First-principles calculations for point defects in solids. Reviews of Modern Physics 86, 253-305 (2014).

43. S. K. Estreicher, Structure and Dynamics of Point Defects in Crystalline Silicon. Physica Status Solidi (B) 217, 513-532 (2000).

44. J. E. Northrup, Effective correlation energy of a Si dangling bond calculated with the local-spin-density approximation. Physical Review B 40, 5875-5878 (Sept. 1989).

45. G. D. Watkins, in Advances in Solid State Physics (Springer Berlin Heidelberg, Berlin, Heidelberg), pp. 163-189.

46. E. H. Poindexter, G. J. Gerardi, M.-E. Rueckel, P. J. Caplan, N. M. Johnson, D. K. Biegelsen, Electronic traps and $\mathrm{P}_{\mathrm{b}}$ centers at the $\mathrm{Si} / \mathrm{SiO}_{2}$ interface: Band-gap energy distribution. Journal of Applied Physics 56, 2844 (1984).

47. E. H. Poindexter, P. J. Caplan, B. E. Deal, R. R. Razouk, Interface states and electron spin resonance centers in thermally oxidized (111) and (100) silicon wafers. Journal of Applied Physics 52, 879-884 (1981).

48. M. Lannoo, The role of dangling bonds in the properties of surfaces and interfaces of semiconductors. Revue de Physique Appliquée 25, 887-894 (1990).

49. J. P. Campbell, P. M. Lenahan, Density of states of $\mathrm{P}_{\mathrm{b} 1} \mathrm{Si} / \mathrm{SiO}_{2}$ interface trap centers. Applied Physics Letters 80, 1945-1947 (2002).

50. R. Entner, Modeling and Simulation of Negative Bias Temperature Instability. 137 (2007).

51. L.- $\AA$. Ragnarsson, P. Lundgren, Electrical characterization of $\mathrm{P}_{\mathrm{b}}$ centers in (100) $\mathrm{Si}_{-} \mathrm{SiO}_{2}$ structures: The influence of surface potential on passivation during post metallization anneal. Journal of Applied Physics 88, 938 (2000).

52. P. Broqvist, A. Alkauskas, A. Pasquarello, Defect levels of dangling bonds in silicon and germanium through hybrid functionals. Physical Review B 78, 075203 (Aug. 2008).

53. A. Alkauskas, P. Broqvist, A. Pasquarello, Defect levels through hybrid density functionals: Insights and applications. Physica Status Solidi (B) Basic Research (2011). 
54. S. R. Schofield, P. Studer, C. F. Hirjibehedin, N. J. Curson, G. Aeppli, D. R. Bowler, Quantum engineering at the silicon surface using dangling bonds. Nature Communications 4, 1649 (Apr. 2013).

55. E. Cartier, J. H. Stathis, D. a. Buchanan, Passivation and depassivation of silicon dangling bonds at the $\mathrm{Si} / \mathrm{SiO}_{2}$ interface by atomic hydrogen. Applied Physics Letters 63, 1510 (1993).

56. A. H. Edwards, Interaction of $\mathrm{H}$ and $\mathrm{H}_{2}$ with the silicon dangling orbital at the $111 \mathrm{Si} / \mathrm{SiO}_{2}$ interface. Physical Review B 44, 1832-1838 (1991).

57. A. Stesmans, Passivation of $\mathrm{P}_{\mathrm{b} 1}$ and $\mathrm{P}_{\mathrm{b} 1}$ interface defects in thermal (100) $\mathrm{Si} / \mathrm{SiO}_{2}$ with molecular hydrogen. Applied Physics Letters 68, 2076 (1996).

58. K. L. Brower, Kinetics of $\mathrm{H}_{2}$ passivation of $\mathrm{P}_{\mathrm{b}}$ centers at the (111) $\mathrm{Si} / \mathrm{SiO}_{2}$ interface. Physical Review B 38, 9657-9666 (1988).

59. J. C. H. Chen, D. Q. Wang, O. Klochan, A. P. Micolich, K. Das Gupta, F. Sfigakis, D. A. Ritchie, D. Reuter, A. D. Wieck, A. R. Hamilton, Fabrication and characterization of ambipolar devices on an undoped AlGaAs/GaAs heterostructure. Applied Physics Letters 100, 2012-2015 (2012).

60. M. G. Borselli, K. Eng, E. T. Croke, B. M. Maune, B. Huang, R. S. Ross, A. A. Kiselev, P. W. Deelman, I. Alvarado-Rodriguez, A. E. Schmitz, M. Sokolich, K. S. Holabird, T. M. Hazard, M. F. Gyure, A. T. Hunter, Pauli spin blockade in undoped $\mathrm{Si} / \mathrm{SiGe}$ two-electron double quantum dots. $A p$ plied Physics Letters 99, 063109 (2011).

61. G. Katsaros, P. Spathis, M. Stoffel, F. Fournel, M. Mongillo, V. Bouchiat, F. Lefloch, A. Rastelli, O. G. Schmidt, S. De Franceschi, Hybrid superconductorsemiconductor devices made from self-assembled SiGe nanocrystals on silicon. Nature Nanotechnology 5, 458-464 (May 2010).

62. M. Brauns, J. Ridderbos, A. Li, W. G. van der Wiel, E. P. A. M. Bakkers, F. A. Zwanenburg, Highly tuneable hole quantum dots in Ge-Si core-shell nanowires. Applied Physics Letters 109, 143113 (2016).

63. K. Horibe, T. Kodera, S. Oda, Lithographically defined few-electron silicon quantum dots based on a silicon-on-insulator substrate. Applied Physics Letters 106, 1-6 (2015).

64. W. H. Lim, F. A. Zwanenburg, H. Huebl, M. Möttönen, K. W. Chan, A. Morello, A. S. Dzurak, Observation of the single-electron regime in a highly tunable silicon quantum dot. Applied Physics Letters 95, 242102 (2009).

65. F. A. Zwanenburg, A. S. Dzurak, A. Morello, M. Y. Simmons, L. C. L. Hollenberg, G. Klimeck, S. Rogge, S. N. Coppersmith, M. A. Eriksson, Silicon quantum electronics. Reviews of Modern Physics 85, 961-1019 (July 2013).

66. R. de Sousa, S. Das Sarma, Theory of nuclear-induced spectral diffusion: Spin decoherence of phosphorus donors in Si and GaAs quantum dots. Physical Review B 68, 115322 (2003). 
67. M. Rashidi, J. A. J. Burgess, M. Taucer, R. Achal, J. L. Pitters, S. Loth, R. A. Wolkow, Time-resolved single dopant charge dynamics in silicon. $\mathrm{Na}$ ture Communications 7, 13258 (2016).

68. A. Laucht, R. Kalra, S. Simmons, J. P. Dehollain, J. T. Muhonen, F. A. Mohiyaddin, S. Freer, F. E. Hudson, K. M. Itoh, D. N. Jamieson, J. C. McCallum, A. S. Dzurak, A. Morello, A dressed spin qubit in silicon. Nature Nanotechnology 12, 61-66 (Oct. 2016).

69. Z. Shi, C. B. Simmons, J. R. Prance, J. K. Gamble, T. S. Koh, Y.-P. Shim, X. Hu, D. E. Savage, M. G. Lagally, M. A. Eriksson, M. Friesen, S. N. Coppersmith, Fast Hybrid Silicon Double-Quantum-Dot Qubit. Physical Review Letters 108, 140503 (Apr. 2012).

70. M. Stewart, N. Zimmerman, Stability of Single Electron Devices: Charge Offset Drift. Applied Sciences 6, 187 (June 2016).

71. B. Van Zeghbroeck, Principles of semiconductor devices. Colorado University (2004).

72. F. Mueller, G. Konstantaras, P. C. Spruijtenburg, W. G. van der Wiel, F. a. Zwanenburg, Electron-Hole Confinement Symmetry in Silicon Quantum Dots. Nano Letters 15, 5336-5341 (Aug. 2015).

73. A. C. Betz, M. F. Gonzalez-Zalba, G. Podd, A. J. Ferguson, Ambipolar quantum dots in intrinsic silicon. Applied Physics Letters 105, 153113 (Oct. 2014).

74. P. Jarillo-Herrero, S. Sapmaz, C. Dekker, L. P. Kouwenhoven, H. S. J. Van Der Zant, Electron-hole symmetry in a semiconducting carbon nanotube quantum dot. Nature 429, 389-392 (2004).

75. J. Güttinger, C. Stampfer, F. Libisch, T. Frey, J. Burgdörfer, T. Ihn, K. Ensslin, Electron-hole crossover in graphene quantum dots. Physical Review Letters 103, 1-4 (2009).

76. H. Pfleiderer, Elementary ambipolar field-effect transistor model. IEEE Transactions on Electron Devices 33, 145-147 (1986).

77. A. Lakhtakia, R. Messier, Sculptured thin films: nanoengineered morphology and optics (SPIE press, 2005), vol. 143.

78. F. Müller, $\mathrm{PhD}$ thesis, University of Twente, June 2015.

79. B. Amin-Ahmadi, H. Idrissi, M. Galceran, M. Colla, J. Raskin, T. Pardoen, S. Godet, D. Schryvers, Effect of deposition rate on the microstructure of electron beam evaporated nanocrystalline palladium thin films. Thin Solid Films 539, 145-150 (July 2013).

80. K. Bordo, H.-G. Rubahn, Effect of Deposition Rate on Structure and Surface Morphology of Thin Evaporated Al Films on Dielectrics and Semiconductors. Materials Science 18, 313-317 (Dec. 2012). 
81. R. L. Puurunen, A Short History of Atomic Layer Deposition: Tuomo Suntola's Atomic Layer Epitaxy. Chemical Vapor Deposition 20, 332-344 (Dec. 2014).

82. G. Dingemans, W. Beyer, M. C. M. van de Sanden, W. M. M. Kessels, Hydrogen induced passivation of $\mathrm{Si}$ interfaces by $\mathrm{Al}_{2} \mathrm{O}_{3}$ films and $\mathrm{SiO}_{2} / \mathrm{Al}_{2} \mathrm{O}_{3}$ stacks. Applied Physics Letters 97, 152106 (Oct. 2010).

83. T. Thorbeck, N. M. Zimmerman, Formation of strain-induced quantum dots in gated semiconductor nanostructures. AIP Advances 5, 087107 (Aug. 2015).

84. H. Kageshima, K. Shiraishi, M. Uematsu, Universal theory of Si oxidation rate and importance of interfacial Si emission. Japanese Journal of Applied Physics, Part 2: Letters 38 (1999).

85. M. Uematsu, H. Kageshima, K. Shiraishi, Microscopic mechanism of thermal silicon oxide growth. Computational Materials Science 24, 229-234 (May 2002).

86. M. Uematsu, H. Kageshima, K. Shiraishi, Interfacial Silicon Emission in Dry Oxidation-the Effect of $\mathrm{H}$ and $\mathrm{Cl}$. Japanese Journal of Applied Physics 41, 2455-2458 (Apr. 2002).

87. K. Yamabe, K. Ohsawa, Y. Hayashi, R. Hasunuma, presented at the ECS Transactions, vol. 19, pp. 427-442.

88. N. T. Yamabe, M. Murata, D. Hojo, Kikuo, $\mathrm{SiO}_{2}$ Surface and $\mathrm{SiO}_{2} / \mathrm{Si}$ Interface Topography Change by Thermal Oxidation. Japanese Journal of Applied Physics 40, 4763 (2001).

89. N. M. Ravindra, J. Narayan, Silicon oxidation and $\mathrm{Si}_{-} \mathrm{SiO}_{2}$ interface of thin oxides. 2 (1987).

90. T. Ohmi, M. Hirayama, A. Teramoto, New era of silicon technologies due to radical reaction based semiconductor manufacturing. Journal of Physics D: Applied Physics 39, R1-R17 (2006).

91. T. Aratani, M. Higuchi, S. Sugawa, E. Ikenaga, J. Ushio, H. Nohira, T. Suwa, A. Teramoto, T. Ohmi, T. Hattori, Angle-resolved photoelectron study on the structures of silicon nitride films and $\mathrm{Si}_{3} \mathrm{~N}_{4} / \mathrm{Si}$ interfaces formed using nitrogen-hydrogen radicals. Journal of Applied Physics 104, 114112 (2008).

92. R. Kuroda, T. Suwa, A. Teramoto, R. Hasebe, S. Sugawa, T. Ohmi, Atomically Flat Silicon Surface and Silicon/Insulator Interface Formation Technologies for (100) Surface Orientation Large-Diameter Wafers Introducing High Performance and Low-Noise Metal-Insulator-Silicon FETs. IEEE Transactions on Electron Devices 56, 291-298 (Feb. 2009).

93. L. Tsetseris, S. T. Pantelides, Oxygen migration, agglomeration, and trapping: Key factors for the morphology of the $\mathrm{Si}_{-} \mathrm{SiO}_{2}$ interface. Physical Review Letters 97, 1-4 (2006). 
94. Y. Schiele, G. Hahn, B. Terheiden, presented at the EU PVSEC Proceedings, pp. 1068-1072.

95. F. Messina, S. Agnello, M. Cannas, A. Parlato, Room Temperature Instability of E' $\gamma$ Centers Induced by $\gamma$ Irradiation in Amorphous $\mathrm{SiO}_{2}$. The Journal of Physical Chemistry A 113, 1026-1032 (Feb. 2009).

96. E. J. Kobetich, R. Katz, Energy Deposition by Electron Beams and $\delta$ Rays. Physical Review 170, 391-396 (June 1968).

97. K. Kanaya, S. Okayama, Penetration and energy-loss theory of electrons in solid targets. Journal of Physics D: Applied Physics 5, 308 (Jan. 1972).

98. C. V. Thompson, Solid-State Dewetting of Thin Films. Annual Review of Materials Research 42, 399-434 (2012).

99. S. Dutta, J. M. Biser, R. P. Vinci, H. M. Chan, Solid State Annealing Behavior of Aluminum Thin Films on Sapphire. 830, 823-830 (2012).

100. D. S. Gardner, P. A. Flinn, Mechanical stress as a function of temperature in aluminum films. IEEE Transactions on Electron Devices 35, 2160-2169 (1988).

101. A. Bierhals, A. G. Aberle, R. Hezel, Improved understanding of thermally activated structural changes in $\mathrm{Al} / \mathrm{SiO}_{x}$ p-Si tunnel diodes by means of infrared spectroscopy. Journal of Applied Physics 83, 1371 (1998).

102. K. Hinode, I. Asano, Y. Homma, Void formation mechanism in VLSI aluminum metallization. IEEE Transactions on Electron Devices 36, 10501055 (1989).

103. Y. A. Bychkov, E. I. Rashba, Oscillatory effects and the magnetic susceptibility of carriers in inversion layers. JETP lett 39, 78 (Nov. 1984).

104. A. Ohtomo, H. Y. Hwang, A high-mobility electron gas at the $\mathrm{LaAlO}_{3} / \mathrm{SrTiO}_{3}$ heterointerface. Nature 427, 423-426 (Jan. 2004).

105. S. T. Pantelides, R. Buczko, M. Ramamoorthy, S. Rashkeev, G. Duscher, S. J. Pennycook, in, ed. by Y. J. Chabal (Springer Berlin Heidelberg, Berlin, Heidelberg, 2001), pp. 193-218.

106. N. H. Thoan, K. Keunen, V. V. Afanas'ev, A. Stesmans, Interface state energy distribution and $\mathrm{P}_{\mathrm{b}}$ defects at $\mathrm{Si}(110) / \mathrm{SiO}_{2}$ interfaces: Comparison to (111) and (100) silicon orientations. Journal of Applied Physics 109, 013710 (2011).

107. T. Mishima, P. Lenahan, A spin-dependent recombination study of radiationinduced $\mathrm{P}_{\mathrm{b} 1}$ centers at the $[001] \mathrm{Si} / \mathrm{SiO}_{2}$ interface. IEEE Transactions on Nuclear Science 47, 2249-2255 (2000).

108. D. K. Schroder, J. A. Babcock, Negative bias temperature instability: Road to cross in deep submicron silicon semiconductor manufacturing. Journal of Applied Physics 94, 1 (2003). 
109. J. P. Campbell, P. M. Lenahan, C. J. Cochrane, A. T. Krishnan, S. Krishnan, Atomic-Scale Defects Involved in the Negative-Bias Temperature Instability. IEEE Transactions on Device and Materials Reliability 7, 540-557 (Dec. 2007).

110. A. R. Stegner, C. Boehme, H. Huebl, M. Stutzmann, K. Lips, M. S. Brandt, Electrical detection of coherent ${ }^{31} \mathrm{P}$ spin quantum states. Nature Physics 2, 835-838 (Dec. 2006).

111. S. Y. Paik, S. Y. Lee, W. J. Baker, D. R. McCamey, C. Boehme, $\mathrm{T}_{1}$ and $\mathrm{T}_{2}$ spin relaxation time limitations of phosphorous donor electrons near crystalline silicon to silicon dioxide interface defects. Physical Review B Condensed Matter and Materials Physics 81, 19-25 (2010).

112. M. Xiao, I. Martin, H. W. Jiang, Probing the spin state of a single electron trap by random telegraph signal. Physical review letters 91, 078301 (2003).

113. M. Xiao, I. Martin, E. Yablonovitch, H. W. Jiang, Electrical detection of the spin resonance of a single electron in a silicon field-effect transistor. Nature 430, 435-439 (July 2004).

114. E. Prati, M. Fanciulli, G. Ferrari, M. Sampietro, Effect of the triplet state on the random telegraph signal in Si n-MOSFETs. Physical Review B 74, 033309 (July 2006).

115. B. S. Aldinger, M. A. Hines, Si(100) Etching in Aqueous Fluoride Solutions: Parallel Etching Reactions Lead to pH-Dependent Nanohillock Formation or Atomically Flat Surfaces. The Journal of Physical Chemistry C 116, 21499-21507 (Oct. 2012).

116. A. Svizhenko, P. W. Leu, K. Cho, Effect of growth orientation and surface roughness on electron transport in silicon nanowires. Physical Review B Condensed Matter and Materials Physics 75, 1-7 (2007).

117. D. M. Fleetwood, S. T. Pantelides, R. D. Schrimpf, Eds., Defects in Microelectronic Materials and Devices (CRC Press, 2008).

118. J. Kim, J.-Y. Ji, J. Kline, J. Tucker, T.-C. Shen, Preparation of atomically clean and flat $\mathrm{Si}(100)$ surfaces by low-energy ion sputtering and lowtemperature annealing. Applied Surface Science 220, 293-297 (Dec. 2003).

119. G. Dingemans, W. M. M. Kessels, Status and prospects of $\mathrm{Al}_{2} \mathrm{O}_{3}$-based surface passivation schemes for silicon solar cells. Journal of Vacuum Science E Technology A: Vacuum, Surfaces, and Films 30, 040802 (July 2012).

120. D. K. Simon, P. M. Jordan, T. Mikolajick, I. Dirnstorfer, On the Control of the Fixed Charge Densities in $\mathrm{Al}_{2} \mathrm{O}_{3}$-Based Silicon Surface Passivation Schemes. ACS Applied Materials and Interfaces 7, 28215-28222 (2015).

121. S. Roberts, P. J. Dobson, Evidence for reaction at the $\mathrm{Al}_{-} \mathrm{SiO}_{2}$ interface. Journal of Physics D: Applied Physics 14, L17-L22 (1981). 
122. I. G. Neizvestnyi, V. A. Gridchin, The use of stressed silicon in MOS transistors and CMOS structures. Russian Microelectronics 38, 71-86 (Mar. 2009).

123. M. Horstmann, A. Wei, T. Kammler, J. Hntschel, H. Bierstedt, T. Feudel, K. Frohberg, M. Gerhardt, A. Hellmich, K. Hempel, J. Hohage, P. Javorka, J. Klais, G. Koerner, M. Lenski, A. Neu, R. Otterbach, P. Press, C. Reichel, M. Trentsch, B. Trui, H. Salz, M. Schaller, H.-J. Engelmann, O. Herzog, H. Ruelke, P. Hubler, R. Stephan, D. Greenlaw, M. Raab, N. Kepler, presented at the IEEE InternationalElectron Devices Meeting, 2005. IEDM Technical Digest. Vol. 00, pp. 233-236.

124. B. Voisin, V.-H. Nguyen, J. Renard, X. Jehl, S. Barraud, F. Triozon, M. Vinet, I. Duchemin, Y.-M. Niquet, S. de Franceschi, M. Sanquer, FewElectron Edge-State Quantum Dots in a Silicon Nanowire Field-Effect Transistor. Nano Letters 14, 2094-2098 (2014).

125. A. Stesmans, Interaction of $\mathrm{P}_{\mathrm{b}}$ defects at the (111) $\mathrm{Si} / \mathrm{SiO}_{2}$ interface with molecular hydrogen: Simultaneous action of passivation and dissociation. Journal of Applied Physics 88, 489 (2000).

126. G. Dingemans, F. Einsele, W. Beyer, M. C. M. van de Sanden, W. M. M. Kessels, Influence of annealing and $\mathrm{Al}_{2} \mathrm{O}_{3}$ properties on the hydrogen-induced passivation of the $\mathrm{Si} / \mathrm{SiO}_{2}$ interface. Journal of Applied Physics 111, 093713 (2012).

127. G. Dingemans, W. M. M. Kessels, Status and prospects of $\mathrm{Al}_{2} \mathrm{O}_{3}$-based surface passivation schemes for silicon solar cells Status and prospects of $\mathrm{Al}_{2} \mathrm{O}_{3}$-based surface passivation schemes for silicon solar cells. 040802, 127 (2015).

128. P. C. Spruijtenburg, J. Ridderbos, F. Mueller, A. W. Leenstra, M. Brauns, A. A. I. Aarnink, W. G. van der Wiel, F. A. Zwanenburg, Single-hole tunneling through a two-dimensional hole gas in intrinsic silicon. Applied Physics Letters 102, 192105 (May 2013).

129. T. D. Ladd, F. Jelezko, R. Laflamme, Y. Nakamura, C. Monroe, J. L. O'Brien, Quantum computers. Nature 464, 45-53 (Mar. 2010).

130. F. Kuemmeth, S. Ilani, D. C. Ralph, P. L. McEuen, Coupling of spin and orbital motion of electrons in carbon nanotubes. Nature 452, 448-452 (Mar. 2008).

131. C. B. Simmons, M. Thalakulam, N. Shaji, L. J. Klein, H. Qin, R. H. Blick, D. E. Savage, M. G. Lagally, S. N. Coppersmith, M. A. Eriksson, Singleelectron quantum dot in $\mathrm{Si} / \mathrm{SiGe}$ with integrated charge sensing. Applied Physics Letters 91, 213103 (2007). 
132. M. G. Borselli, K. Eng, E. T. Croke, B. M. Maune, B. Huang, R. S. Ross, A. A. Kiselev, P. W. Deelman, I. Alvarado-Rodriguez, A. E. Schmitz, M. Sokolich, K. S. Holabird, T. M. Hazard, M. F. Gyure, A. T. Hunter, Pauli spin blockade in undoped $\mathrm{Si} / \mathrm{SiGe}$ two-electron double quantum dots. $A p$ plied Physics Letters 99, 063109 (2011).

133. G. P. Lansbergen, R. Rahman, C. J. Wellard, I. Woo, J. Caro, N. Collaert, S. Biesemans, G. Klimeck, L. C. L. Hollenberg, S. Rogge, Gate-induced quantum-confinement transition of a single dopant atom in a silicon FinFET. Nature Physics 4, 656-661 (2008).

134. M. Fuechsle, J. A. Miwa, S. Mahapatra, H. Ryu, S. Lee, O. Warschkow, L. C. L. Hollenberg, G. Klimeck, M. Y. Simmons, A single-atom transistor. Nature Nanotechnology 7, 242-246 (2012).

135. E. Prati, M. De Michielis, M. Belli, S. Cocco, M. Fanciulli, D. KotekarPatil, M. Ruoff, D. P. Kern, D. a. Wharam, J. Verduijn, G. C. Tettamanzi, S. Rogge, B. Roche, R. Wacquez, X. Jehl, M. Vinet, M. Sanquer, Few electron limit of n-type metal oxide semiconductor single electron transistors. Nanotechnology 23, 215204 (June 2012).

136. J. J. L. Morton, D. R. McCamey, M. A. Eriksson, S. A. Lyon, Embracing the quantum limit in silicon computing. Nature 479, 345-353 (Nov. 2011).

137. J. J. Pla, K. Y. Tan, J. P. Dehollain, W. H. Lim, J. J. L. Morton, D. N. Jamieson, A. S. Dzurak, A. Morello, A single-atom electron spin qubit in silicon. Nature 489, 541-545 (Sept. 2012).

138. K. C. Nowack, F. H. L. Koppens, Y. V. Nazarov, L. M. K. Vandersypen, Coherent Control of a Single Electron Spin with Electric Fields. Science 318, 1430-1433 (Nov. 2007).

139. E. A. Laird, C. Barthel, E. I. Rashba, C. M. Marcus, M. P. Hanson, A. C. Gossard, Hyperfine-Mediated Gate-Driven Electron Spin Resonance. Physical Review Letters 99, 246601 (Dec. 2007).

140. M. Pioro-Ladrière, T. Obata, Y. Tokura, Y.-S. Shin, T. Kubo, K. Yoshida, T. Taniyama, S. Tarucha, Electrically driven single-electron spin resonance in a slanting Zeeman field. Nature Physics 4, 776-779 (Oct. 2008).

141. S. Nadj-Perge, S. M. Frolov, E. P. A. M. Bakkers, L. P. Kouwenhoven, Spin-orbit qubit in a semiconductor nanowire. Nature 468, 1084-1087 (Apr. 2011).

142. V. S. Pribiag, S. Nadj-Perge, S. M. Frolov, J. W. G. van den Berg, I. van Weperen, S. R. Plissard, E. P. A. M. Bakkers, L. P. Kouwenhoven, Electrical control of single hole spins in nanowire quantum dots. Nature Nanotechnology 8, 170-174 (Feb. 2013).

143. R. Li, F. E. Hudson, A. S. Dzurak, A. R. Hamilton, Single hole transport in a silicon metal-oxide-semiconductor quantum dot. Applied Physics Letters 103, 163508 (Apr. 2013). 
144. C. C. Escott, F. A. Zwanenburg, A. Morello, Resonant tunnelling features in quantum dots. Nanotechnology 21, 274018 (July 2010).

145. P. C. Spruijtenburg, S. V. Amitonov, F. Mueller, W. G. van der Wiel, F. A. Zwanenburg, Passivation and characterization of charge defects in ambipolar silicon quantum dots. Scientific Reports 6, 38127 (2016).

146. W. G. van der Wiel, S. De Franceschi, J. M. Elzerman, T. Fujisawa, S. Tarucha, L. P. Kouwenhoven, Electron transport through double quantum dots. Rev. Mod. Phys. 75, 1-22 (Dec. 2002).

147. A. P. Higginbotham, F. Kuemmeth, M. P. Hanson, A. C. Gossard, C. M. Marcus, Coherent Operations and Screening in Multielectron Spin Qubits. Physical Review Letters 112, 026801 (Jan. 2014).

148. C. B. Simmons, M. Thalakulam, B. M. Rosemeyer, B. J. Van Bael, E. K. Sackmann, D. E. Savage, M. G. Lagally, R. Joynt, M. Friesen, S. N. Coppersmith, M. A. Eriksson, Charge Sensing and Controllable Tunnel Coupling in a Si/SiGe Double Quantum Dot. Nano Letters 9, 3234-3238 (Sept. 2009).

149. M. G. Borselli, K. Eng, R. S. Ross, T. M. Hazard, K. S. Holabird, B. Huang, A. A. Kiselev, P. W. Deelman, L. D. Warren, I. Milosavljevic, A. E. Schmitz, M. Sokolich, M. F. Gyure, A. T. Hunter, Undoped accumulationmode Si/SiGe quantum dots. Nanotechnology 26, 375202 (Sept. 2015).

150. J. van der Heijden, J. Salfi, J. A. Mol, J. Verduijn, G. C. Tettamanzi, A. R. Hamilton, N. Collaert, S. Rogge, Probing the Spin States of a Single Acceptor Atom. Nano Letters 14, 1492-1496 (Mar. 2014).

151. M. Pierre, R. Wacquez, X. Jehl, M. Sanquer, M. Vinet, O. Cueto, Singledonor ionization energies in a nanoscale CMOS channel. Nature Nanotechnology 5, 133-137 (Feb. 2010).

152. D. Jovanovic, J. P. Leburton, H. Chang, R. Grundbacher, I. Adesida, Disorderinduced resonant tunneling in planar quantum-dot nanostructures. Physical Review B 50, 5412-5419 (Aug. 1994).

153. M. Hofheinz, X. Jehl, M. Sanquer, G. Molas, M. Vinet, S. Deleonibus, Individual charge traps in silicon nanowires. The European Physical Journal B 54, 299-307 (Dec. 2006).

154. E. Prati, M. Hori, F. Guagliardo, G. Ferrari, T. Shinada, Anderson - Mott transition in arrays of a few dopant atoms in a silicon transistor. Nature Nanotechnology 7, 443-447 (2012).

155. F. Mueller, G. Konstantaras, P. C. Spruijtenburg, W. G. van der Wiel, F. A. Zwanenburg, Electron-Hole Confinement Symmetry in Silicon Quantum Dots. Nano Letters 15, 5336-5341 (Aug. 2015).

156. Y. Y. Kim, P. M. Lenahan, Electron-spin-resonance study of radiationinduced paramagnetic defects in oxides grown on (100) silicon substrates. Journal of Applied Physics 64, 3551 (1988). 
157. E. A. Laird, J. M. Taylor, D. P. Divincenzo, C. M. Marcus, M. P. Hanson, A. C. Gossard, Coherent spin manipulation in an exchange-only qubit. Physical Review B - Condensed Matter and Materials Physics 82, 1-6 (2010).

158. E. Kawakami, T. Jullien, P. Scarlino, D. R. Ward, D. E. Savage, M. G. Lagally, V. V. Dobrovitski, M. Friesen, S. N. Coppersmith, M. A. Eriksson, L. M. K. Vandersypen, Gate fidelity and coherence of an electron spin in an Si/SiGe quantum dot with micromagnet. Proceedings of the National Academy of Sciences 113, 11738-11743 (Oct. 2016).

159. Y. Kato, R. C. Myers, D. C. Drisoll, a. C. Gossard, J. Levy, D. D. Awschalom, Gigahertz Electron Spin Manipulation Using Voltage-Controlled g-Tensor Modulation. Science 299, 1201 (2003).

160. R. Maurand, X. Jehl, D. Kotekar-Patil, A. Corna, H. Bohuslavskyi, R. Laviéville, L. Hutin, S. Barraud, M. Vinet, M. Sanquer, S. De Franceschi, A CMOS silicon spin qubit. Nature Communications 7, 13575 (Nov. 2016).

161. J. Salfi, M. Tong, S. Rogge, D. Culcer, Quantum computing with acceptor spins in silicon. Nanotechnology 27, 244001 (June 2016).

162. M. Brauns, J. Ridderbos, A. Li, E. P. A. M. Bakkers, F. A. Zwanenburg, Electric-field dependent g-factor anisotropy in Ge-Si core-shell nanowire quantum dots. Phys. Rev. B 93, 121408 (Mar. 2016).

163. J. Buckley, B. De Salvo, D. Deleruyelle, M. Gely, G. Nicotra, S. Lombardo, J. Damlencourt, P. Hollinger, F. Martin, S. Deleonibus, Reduction of fixed charges in atomic layer deposited $\mathrm{Al}_{2} \mathrm{O}_{3}$ dielectrics. Microelectronic Engineering 80, 210-213 (June 2005).

164. O. Malkoc, P. Stano, D. Loss, Optimal geometry of lateral GaAs and Si/SiGe quantum dots for electrical control of spin qubits. Physical Review B - Condensed Matter and Materials Physics 93, 1-12 (2016).

165. M. Ciorga, A. S. Sachrajda, P. Hawrylak, C. Gould, P. Zawadzki, S. Jullian, Y. Feng, Z. Wasilewski, Addition spectrum of a lateral dot from Coulomb and spin-blockade spectroscopy. Physical Review B 61, R16315 (2000).

166. C. Gould, P. Hawrylak, A. Sachrajda, Y. Feng, P. Zawadzki, Z. Wasilewski, Correlations effects in few-electron quantum dots between $\mathrm{n}=2$ and 1 . Physica E: Low-dimensional Systems and Nanostructures 6, 461-465 (2000).

167. G. M. Jones, B. H. Hu, C. H. Yang, M. J. Yang, Y. B. Lyanda-Geller, Enhancement-mode quantum transistors for single electron spin. Physica E: Low-Dimensional Systems and Nanostructures 34, 612-615 (2006).

168. N. M. Zimmerman, C.-H. Yang, N. Shyan Lai, W. Han Lim, A. S. Dzurak, Charge offset stability in Si single electron devices with Al gates. Nanotechnology 25, 405201 (2014).

169. W. G. van der Wiel, S. De Franceschi, J. M. Elzerman, T. Fujisawa, S. Tarucha, L. P. Kouwenhoven, Electron transport through double quantum dots. Reviews of Modern Physics 75, 1-22 (Dec. 2002). 
170. W. H. Lim, C. H. Yang, F. A. Zwanenburg, A. S. Dzurak, Spin filling of valley-orbit states in a silicon quantum dot. Nanotechnology 22, 335704 (Aug. 2011).

171. C. H. Yang, A. Rossi, R. Ruskov, N. S. Lai, F. A. Mohiyaddin, S. Lee, C. Tahan, G. Klimeck, A. Morello, A. S. Dzurak, Spin-valley lifetimes in a silicon quantum dot with tunable valley splitting. Nature Communications 4, 1-8 (1).

172. F. Mueller, R. N. Schouten, M. Brauns, T. Gang, W. H. Lim, N. S. Lai, A. S. Dzurak, W. G. van der Wiel, F. A. Zwanenburg, Printed circuit board metal powder filters for low electron temperatures. Review of scientific instruments 84, 44706 (2013).

173. S. R. Schofield, P. Studer, C. F. Hirjibehedin, N. J. Curson, G. Aeppli, D. R. Bowler, Quantum engineering at the silicon surface using dangling bonds. Nature communications 4, 1649 (2013).

174. D. M. Zajac, T. M. Hazard, X. Mi, K. Wang, J. R. Petta, D. M. Zajac, T. M. Hazard, X. Mi, K. Wang, J. R. Petta, A reconfigurable gate architecture for $\mathrm{Si} / \mathrm{SiGe}$ quantum dots A reconfigurable gate architecture for $\mathrm{Si} / \mathrm{SiGe}$ quantum dots. 223507, 1-5 (2016).

175. P. C. Spruijtenburg, cryo-attenuation, https ://github.com/wakass/ cryo-attenuation, 2017. 
Appendices 



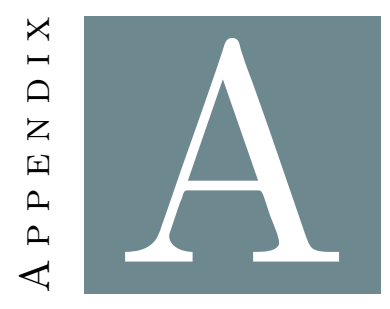

\section{Design of high-frequency transmission lines for low electron temperature}

The goal of doing experiments in a cryogenic environment is to reduce thermal broadening of the Fermi-Dirac distribution. The cryostat bath (phonon) temperature $T_{\text {bath }}$ can be as low as several $\mathrm{mK}$ in modern dilution refrigerators. The thermal energy of the electron gas $E_{e}=k_{B} T_{e}$, often used in transported measurements, does not have to be equal to $T_{b a t h}$ and in fact can be much larger than $T_{\text {bath }}$ if proper care is not taken to thermalise the electrons. For low-frequency DC transport measurements the electrons can be thermalised using metal powder filters.

Thermalising the high-frequency signals to achieve an as low as possible electron temperature $T_{e}$ and obtain as little thermal broadening as possible is the goal of this section. For high-frequency measurements, the signals are transported along coaxial transmission lines to the device under test (DUT). Thermalizing the electrons in coaxial lines can, at present, only be done by attenuating the signal using a chain of attenuators. These elements are placed at a cryogenic stage (which is at temperature $T_{n}$ ) in a cryogenic refrigerator and attenuate the microwaves using a resistive network.

The thermal noise generated at resistive elements is called Johnson-Nyquist noise and can essentially be considered as black-body radiation. It can be modelled as an ideal resistor with an average noise voltage $\bar{v}_{n}^{2}=4 k_{B} T R$ in series or by 
considering an ideal resistor with a current noise current generated in parallel with a noise power $P=k_{B} T \Delta f$.

The high-frequency input at the start of the chain can be split up into two power components, the signal power $P_{0}^{s}$ and the noise power $P_{0}=k_{B} T_{0}$. Passing an high-frequency signal through an attenuator has the following effects. The signal and the noise are both attenuated, but at each stage thermal noise of $P=k_{B} T_{n}$ is also added. This leads to the following relations:

$$
\begin{aligned}
& P_{n}^{s}=P_{n-1}^{s} G_{n} \\
& P_{n}^{n}=P_{n-1}^{n} G_{n}+k_{B} T_{n} .
\end{aligned}
$$

The electron temperature at the DUT is then easily calculated using $T_{e}(N)=$ $P_{N}^{n} / k_{B}$

How noise and signal powers are attenuated is schematically depicted for a 3stage problem in Fig. A.1.

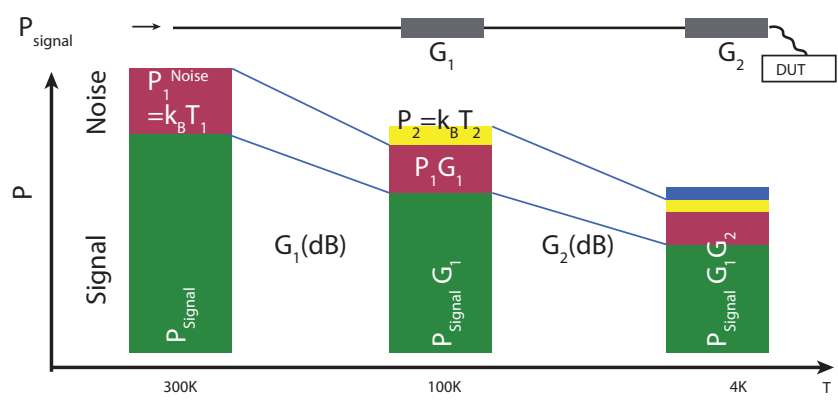

Figure A.1: Schematic representation of attenuation of a signal and thermal noise in a two-attenuator coaxial line.

The dissipation of power at each stage is determined by the power arriving at that stage and the attenuation $G$ present there: $P_{\text {diss }}=\left(P_{n-1}^{s}+P_{n-1}^{n}\right) *\left(1-G_{n}\right)$. The dissipated power ideally does not exceed the cooling power of that cryogenic stage, which would lead to heating.

Designing an attenuation chain for high-frequency in cryogenic setups then becomes a minimization problem with constraints. The constraints being:

1. A maximum input power for each stage due to dissipation.

2. A maximum electron temperature desired, thus keeping the attenuation of the signal also as low as possible.

The optimized values for the attenuators for the particular setup in a Triton 200 dilution refrigerator is found in Appendix A. One coaxial line was optimized for an electron temperature of $T_{e}=53 \mathrm{mK}$, the other for $T_{e}=152 \mathrm{mK}$. 
Table A.1: Chosen attenuator values for the Triton 200 dilution refrigerator, for two separate resultant electron temperatures $T_{e}$.

\begin{tabular}{c|c|c}
\hline Stage & $T_{e} 53 \mathrm{mK}$ & $T_{e} 152 \mathrm{mK}$ \\
\hline PT1, 46 K & $-1 \mathrm{~dB}$ & $-1 \mathrm{~dB}$ \\
PT2, 3.2 K & $-9 \mathrm{~dB}$ & $-10 \mathrm{~dB}$ \\
still, $0.8 \mathrm{~K}$ & $-25 \mathrm{~dB}$ & $-25 \mathrm{~dB}$ \\
100mk, 100 mK & $-6 \mathrm{~dB}$ & $-3 \mathrm{~dB}$ \\
mixing chamber, $22 \mathrm{mK}$ & $-10 \mathrm{~dB}$ & $-6 \mathrm{~dB}$
\end{tabular}

(a)

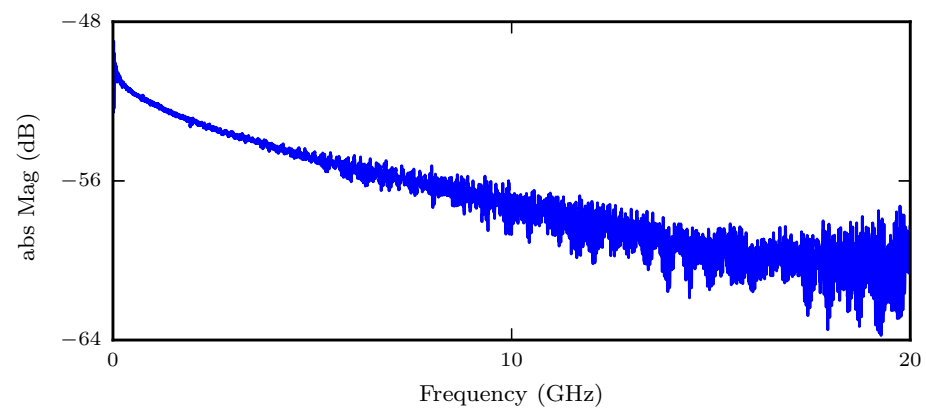

(b)

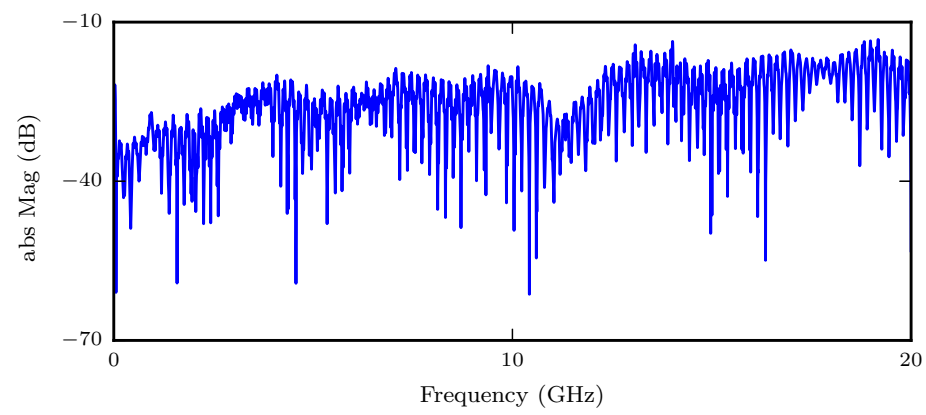

Figure A.2: Characterization of installed microwave tranmission line with design temperature of $53 \mathrm{mK}$. (a) shows the transmittance $S_{21}$. (b) shows the reflectance $S_{11}$.

The optimization problem is solved in a minimization algorithm, and is available online. 



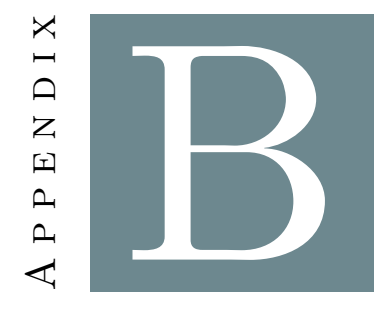

\section{g-tensor anisotropy}

\section{B.1 simple Gaussian fitting method}

Shown in Fig. B.] is an example of the simple Gaussian fitting method. The final conductance curve consists of 4 separate Gaussians, each representing a level as it transitions the bias window. The separate Gaussians are constrained to facilitate better convergence. Specifically, the width of each Gaussian of the first peak-pair is constrained to be equal and varied, while the amplitude is separately defined and varied. Meanwhile, the width of each Gaussian of the second peak-pair is fixed to separate values and not varied, while their amplitudes are linked and varied. Further constraints fix each peak-pair to a specific region in $V_{S D}$.

\section{B.2 parametric fitting method}

Shown in Appendix $\mathbb{B . 2}($ top left panel) is the conductance, as predicted by the model for the initial parameters. Lines in Appendix $\mathbb{B} .2$ (top right panel) indicate the center of the constituent Gaussian peaks of these initial parameters through the data. Appendix B.2(bottom left panel) shows the conductance, as given by the model with the fitted parameters. The solid lines through the data in Appendix B.2(bottom right panel) again indicate the centers of the constituent gaussian peaks. 
(a)

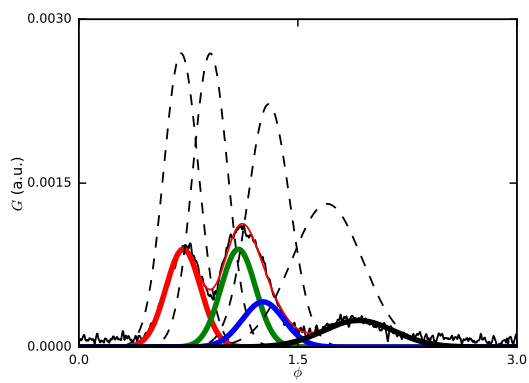

(b)

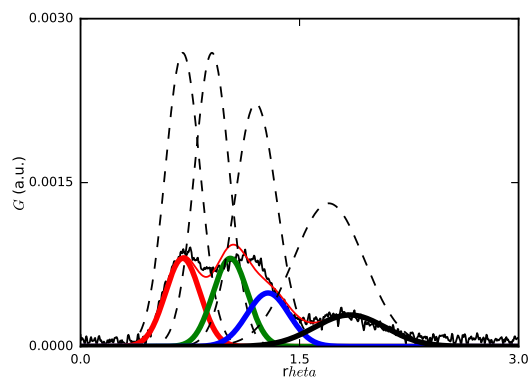

(c)

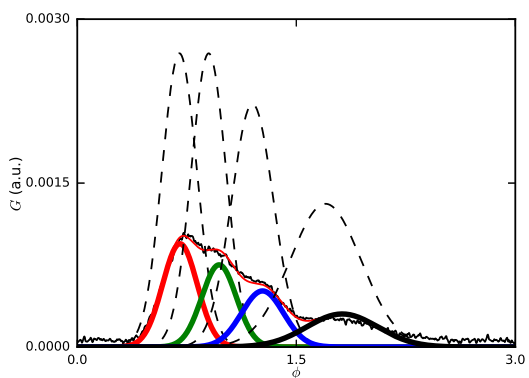

(d)

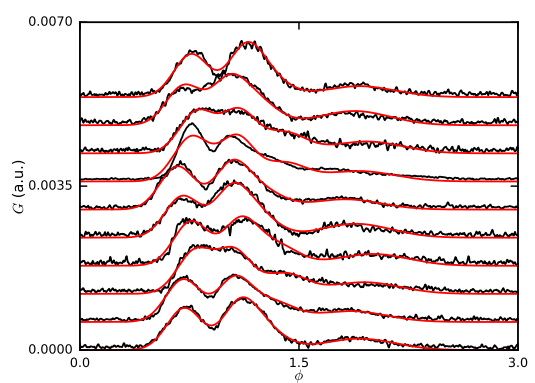

(e)

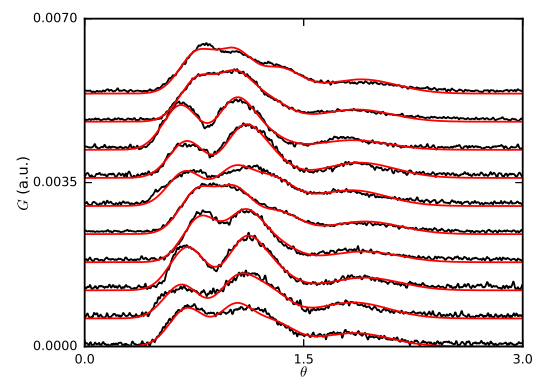

(f)

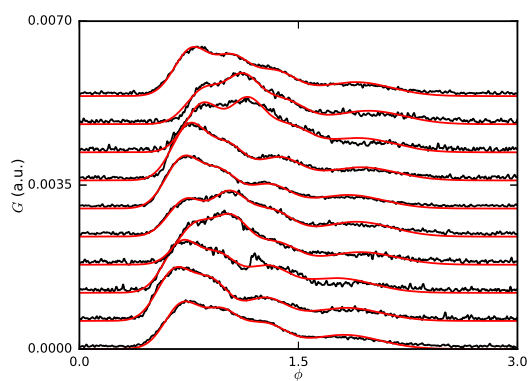

Figure B.1: Gaussian procedure fit for all 3 high-symmetry directions. (a), (d) the zy-plane, (b)(e) the yx-plane, and (c) (f) the zx-plane.

\section{B.3 Uncorrected anisotropy data accross principal planes}




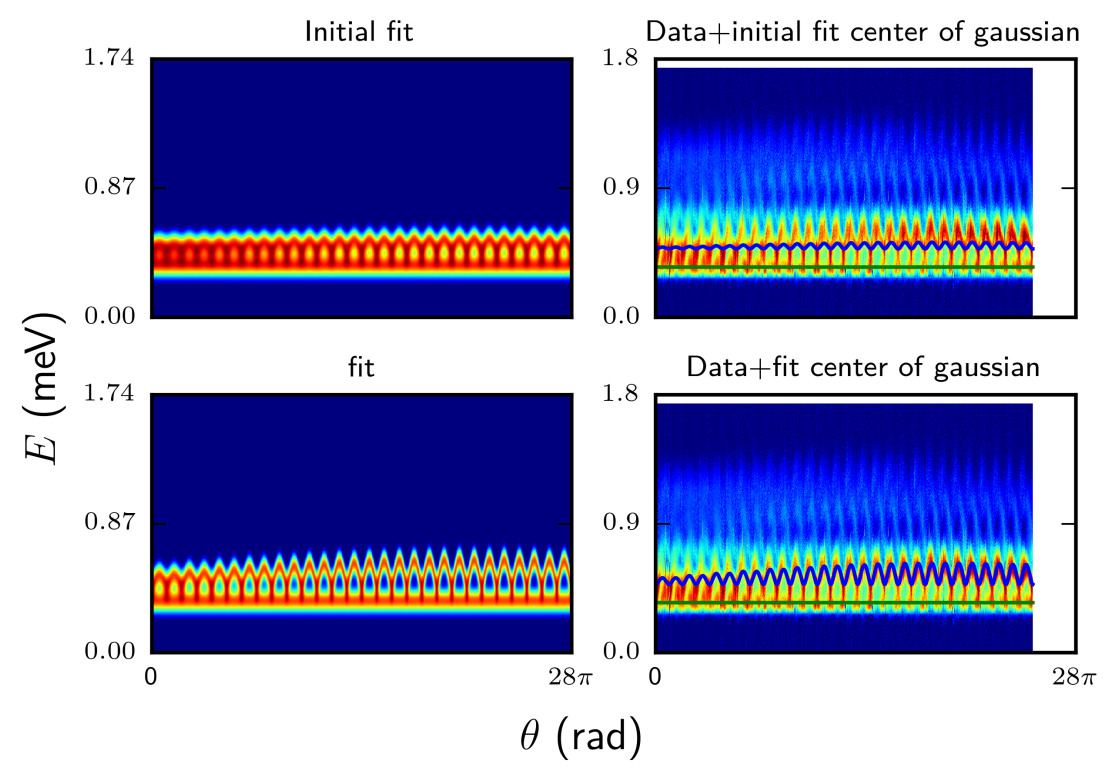

Figure B.2: Example of full parametric fitting procedure. 

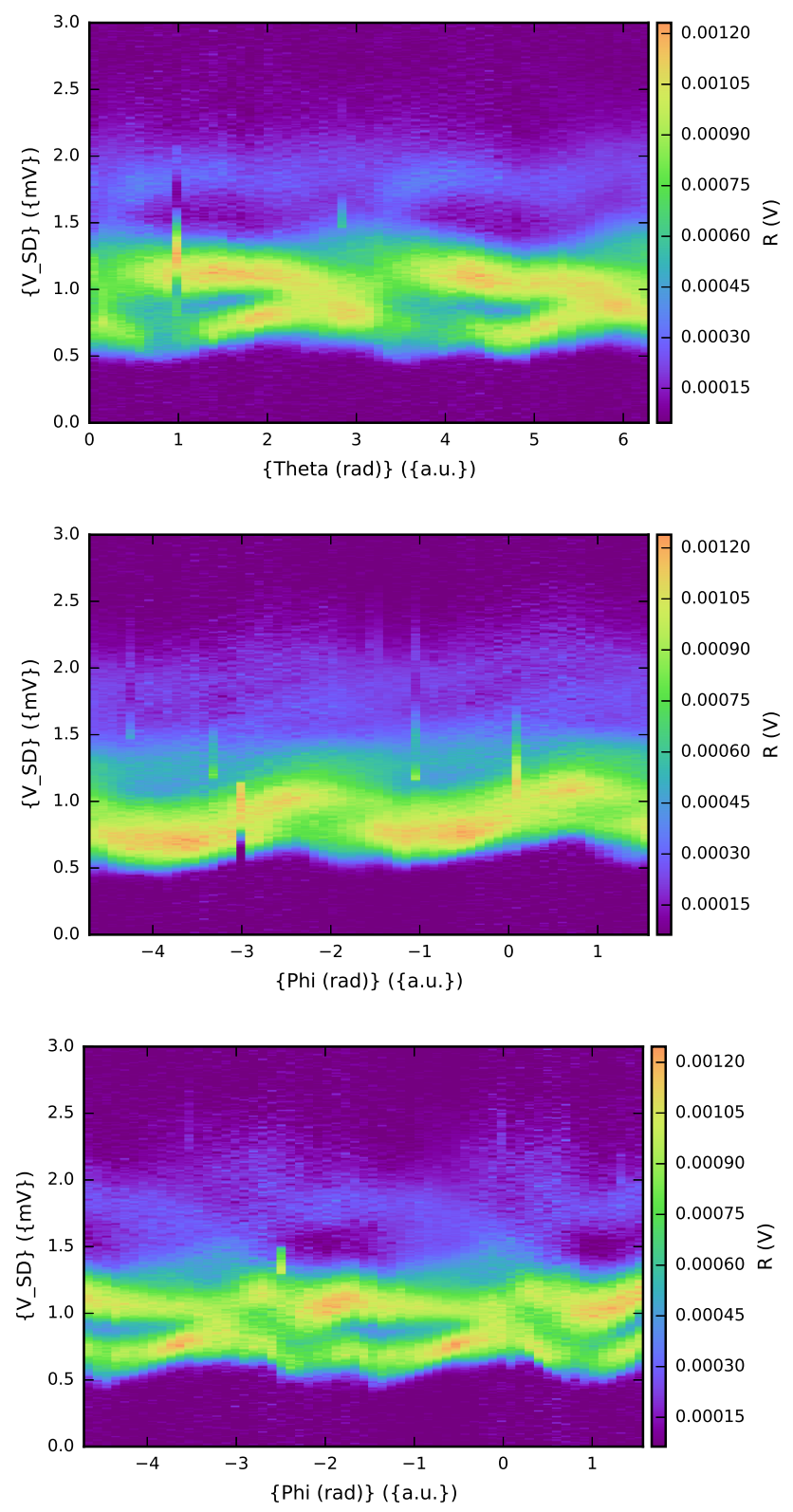

Figure B.3: Conductivity of last-visible transition as a function of rotation of the magnetic field in (a) the yx-plane (a) the zx-plane and (a) in the zy-plane. 


\section{Summary}

Spurred on by the promise of a quantum "speed-up" for certain classes of computational problems, interest in quantum computation has seen a great surge over the past decades. Many approaches have been tried, with a couple of systems vying for a place as a scalable universal quantum computer. One of these approaches is confining carriers to such a small region in a semiconductor, that quantum properties become observable. These "quantum dots" have their best properties in a material with little to no hyperfine-interaction. To this end, silicon has been at the forefront as a very interesting material, as it can be isotopically purified to contain a very low concentration of non-zero nuclear spin isotopes. This has garnered a lot of success in achieving long spin lifetimes. Work thusfar has primarily been focused on electrons as the carriers of spin and charge. However, spin and charge can also be carried by holes, which can be typified as the absence of a single electron in a sea of electrons. In this thesis then, we focus on holes.

The first step in creating quantum dots in semiconductors is the necessity of control over the confinement potential. However, defects, coming about by disorder or other fabricational issues are of great influence on quantum dot formation. This particular thesis concerns itself with the properties of hole quantum dots under the influence of, and the charge transport through, these defects. We explore the origin of these defects, and devise methods of eliminating them.

Chapter 『 concerns the theoretical concepts governing quantum dot transport measurements. We posit single-electron tunneling and the constant interaction model. We discuss what the band diagram in silicon tells us about the behaviour of holes and deduce how the spin-orbit interaction leads to heavy-hole and lighthole characteristics. For holes in quantum wells we find that holes are predicted to have an anisotropic g-tensor. We then discuss the theoretical concepts underlying point defects such as $\mathrm{P}_{\mathrm{b}}$ centers.

Chapter $\mathbf{3}$ examines the entire heterostructure, as it is used in creating electrostatically defined quantum dots. We relate three properties of these heterostructures to the performance of quantum dots: materials, interfaces, and morphology. We find that the ability to passivate defects at the $\mathrm{Si} / \mathrm{SiO} 2$ interface hinges primarily on controlling the dewetting at higher temperatures. We introduce an ALD-grown $\mathrm{Al}_{2} \mathrm{O}_{3}$ layer, which strikes two birds with one stone. The layer both provides hydrogen for the annealing process and prevents dewetting. We also find 
that by supplanting $\mathrm{Al}$ by $\mathrm{Pd}$ as the electrode material, the formation of an interfacial layer between the metal and the oxide is prevented. This can be seen in TEM images. The amount of two-level fluctuators, present in glassy systems accompanying this interface layer, should therefore also be diminished. We further show that the introduction of $\mathrm{Al}_{2} \mathrm{O}_{3}$ introduces a layer of negative fixed charge, which can be eliminated or controlled by exposure to a UV-ozone oxidation process.

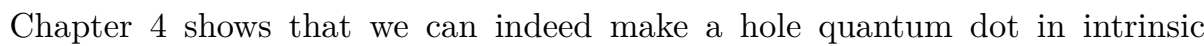
silicon, by demonstrating single-hole tunneling through a two-dimensional hole gas. We show resonant tunneling features of Coulomb islands highly coupled to single controlling gates. The Coulomb islands are caused by local potential fluctuations, most likely due to impurities or charge traps in the $\mathrm{SiO}_{2}$, or at the interface of $\mathrm{Si}$ and $\mathrm{SiO}_{2}$. Silicon is known for being extremely sensitive to disorder, owing to the large effective mass of the charge carriers, which is even higher for holes than for electrons.

Chapter $\mathbf{5}$ sets out to improve upon these results and we investigate defects in the heterostructure. Using $\mathrm{Al}_{2} \mathrm{O}_{3}$ grown by atomic-layer deposition in an annealing process, we passivate the majority of the electrically active defects. Using this oxide, and the passivating properties of the hydrogen contained therein, we are able to create intentional quantum dots of at least $180 \mathrm{~nm}$. These quantum dots show many charge transitions, indicating the low level of disorder in the devices. The versatility of our ambipolar architecture allows us to investigate the amphoteric behaviour of a remaining charge defect, which has states $\approx 10 \mathrm{meV}$ above the valence band, and below the conduction band.

Chapter [ $\mathbf{0}$ extends on our new-found ability to anneal defects and create lowdisorder devices. In this chapter, the spin-orbit interaction starts to play a more important role, as it is responsible for the prediction that hole spins are expected to be preferentially oriented in $2 \mathrm{D}$ quantum wells. We focus on the behaviour of the g-tensor in a rotating magnetic field, and extract the anisotropy of the gtensor. We do this by studying the Zeeman splitting in a magnetic field capable of rotating $360^{\circ}$ over all 3 degrees of freedom. Using two methods of fitting the data to our model, we extract similar anisotropies for the g-tensor $\left(g_{\|}^{*} \approx 2.2, g_{\perp}^{*} \approx 4\right)$, indicating that the g-factor is roughly twice as strong out-of-plane than in-plane. This is consistent with the prediction that light-holes and heavy-holes are oriented preferentially in a 2D quantum well.

In Chapter $\square$ a proof-of-principle of a single-layer depletion-mode hole quantum dot is demonstrated. The depletion architecture has several advantages, such as optical accessibility and having areas of the device free from potentially damaging exposure to electron beam lithography. The negative fixed charge in ALD-grown $\mathrm{Al}_{2} \mathrm{O}_{3}$ enables the operation in depletion-mode by inducing a $2 \mathrm{D}$ hole gas. Equal coupling of charge transitions to adjacent gates indicates that an intentional quantum dot is formed in between them. We are then able to tune the single quantum dot to a double-quantum dot regime by using an interdot coupling gate. Characterization of the charge-offset stability of this device indicates that the device 
is extremely stable, with the charge-offset stability having an upper bound at $Q_{0}=0.04 e$ and a lower bound of $Q_{0}=0.005 e$. This compares favourably to previously known results for all-Si based devices $\left(Q_{0}<0.01 e\right)$ and Al-based devices $\left(Q_{0}<0.15 e\right)$.

While holes are promising candidates for quantum computing, several hurdles have to be overcome in order to exploit their properties. The $\mathrm{Si} / \mathrm{SiO} 2$ interface is known to have many types of defects. These have to be dealt with or avoided altogether. This can be done by annealing, or by switching to a different insulator material. In this regard the $\mathrm{Si} / \mathrm{SiGe}$ system appears to be a good candidate. These, and certainly many other improvements which this thesis has touched upon, could lead to one day possibly making a truly scalable quantum technology based on silicon. 



\section{Samenvatting}

Aangewakkerd door de belofte van een exponentiële "versnelling" voor bepaalde klassen van computationele problemen heeft kwantumcomputatie een grote opleving gezien in de laatste paar decennia. Veel methodes zijn al geprobeerd, met een paar die nog in de race zijn voor een schaalbare kwantumcomputer. Een van deze methodes benadert het probleem door ladingsdragers tot zulk een klein stukje halfgeleider te beperken, dat kwantumverschijnselen meetbaar worden. Deze "kwantum dots" hebben hun beste eigenschappen in een materiaal met weinig tot geen hyperfijn-interacties. Teneinde dit te bereiken heeft silicium veel voordelen, gezien dit isotopisch kan worden verrijkt zodat het een zeer lage concentratie van isotopen met nucleaire spin bevat. Dit heeft geleid tot succesvolle experimenten met zeer lange levensduur voor spin. Tot dusver heeft het veld zich primair gericht op elektronen als de dragers van ladings en spin. Echter kan lading en spin ook gedragen worden door gaten, die zich het beste laten typeren als de absentie van een elektron in een zee van elektronen. In dit proefschrift leggen we ons dan ook toe op gaten.

Om de eerste stap in het creëren van kwantum dots in halfgeleiders te zetten, is het noodzakelijk de opsluitings-potentiaal te beheersen. Echter zijn defecten, die zich manifesteren als gevolg van wanorde en andere factoren in fabricage van grote invloed op de vorming van kwantum dots. Dit proefschrift legt de nadruk verder op de eigenschappen van gaten onder invloed van, en transport door, deze defecten. We verkennen de oorsprong van deze defecten, en bedenken methodes om deze weg te nemen.

Hoofdstuk $\nabla$ beschrijft de theoretische concepten die ten grondslag liggen aan het meten van transport door kwantum dots. We poneren enkel-elektron tunnelgedrag en het constante-interactie model. Daarna behandelen we hoe het bandendiagram het gedrag van gaten in silicium beschrijft en leiden we af hoe de spin-baan koppeling er voor zorgt dat zware- en lichte-gaten ontstaan. Hierna beschrijven we hoe voor gaten in kwantum putten een anisotrope g-factor wordt voorspeld. Vervolgens bespreken we de theoretische concepten die ten grondslag liggen aan puntdefecten, zoals $\mathrm{P}_{\mathrm{b}}$ centers.

Hoofdstuk [ bekijkt de gehele heterostructuur, zoals die wordt gebruikt in het creëren van elektrostatisch gedefinieerde kwantum dots. We relateren drie eigenschappen in deze heterostructuren aan de prestaties van kwantum dots: materi- 
alen, grensvlakken, en morfologie. We ontdekken dat het vermogen defecten aan het $\mathrm{Si} / \mathrm{SiO}_{2}$ grensvlak te passiveren voornamelijk afhangt van het in toom houden van het verliezen van cohesie in de laag bij hogere temperatuur. We introduceren een $\mathrm{Al}_{2} \mathrm{O}_{3}$ laag gegroeid met atomaire-laag depositie, welke twee vliegen in een klap slaat. De laag levert waterstof voor het uitgloeings-proces en voorkomt dat de onderliggende laag zijn cohesie verliest. Ook komen we tot de conclusie dat door het aluminium van de bestaande elektrodes door palladium te vervangen, de vorming van een grensvlak-laag tussen het metaal en het onderliggende oxide wordt voorkomen. Dit wordt waargenomen in transmissie elektronen microscopie. Hiermee zou ook de frequentie waarmee twee-niveau fluctuatoren voorkomen, dat gepaard gaat met glasachtige grensvlaklagen, moeten zijn verminderd. We laten verder in dit hoofdstuk zien dat de $\mathrm{Al}_{2} \mathrm{O}_{3}$ vaste negative lading bevat, welke we kunnen controleren en uiteindelijk weghalen door middel van een UV-ozon oxidatieproces.

Hoofdstuk 四laat zien dat we daadwerkelijk gaten kwantum dots in intrinsiek silicium kunnen maken. We laten tunnelgedrag van enkele gaten zien door een tweedimensionaal gatengas, en resonant tunnelgedrag door Coulomb-eilanden die sterk gekoppeld zijn aan specifieke elektrodes. De Coulomb-eilanden worden veroorzaakt door fluctuaties in de potentiaal, wat op zijn beurt zeer waarschijnlijk wordt veroorzaakt door onzuiverheden of ladingsvallen in het $\mathrm{SiO}_{2}$, of aan het grensvlak tussen $\mathrm{Si}$ en $\mathrm{SiO}_{2}$. Het is bekend van silicium dat het gevoelig is voor wanorde, gezien de hoge effective massa van de ladingsdragers. Voor gaten is deze zelfs hoger dan voor elektronen.

Hoofdstuk gaat vervolgens erop uit om deze resultaten te verbeteren, en we onderzoeken defecten in de heterostructuur. Door het gebruik van $\mathrm{Al}_{2} \mathrm{O}_{3}$ dat gegroeid is met atomaire-laag depositie in een uitgloeings-proces, kunnen we de meerderheid van elektrisch actieve defecten passiveren. Met dit oxide en de passiverende eigenschappen van het waterstof dat daarin aanwezig is, konden we kwantum dots maken van minstens $180 \mathrm{~nm}$. Deze kwantum dots laten vele ladingsovergangen zien wat de lage mate van wanorde aantoont. De veelzijdigheid van onze ambipolaire architectuur stelt ons vervolgens in staat de amfoterische aard van een overgebleven ladingsdefect te onderzoeken. Deze had energieniveaus $\approx 10 \mathrm{meV}$ boven de valentieband, en even zo ver onder de conductieband.

Hoofdstuk [ bast ons nieuwe vermogen om defecten te passiveren toe om kwantum dots met lage wanorde te maken. In dit hoofdstuk speelt de spin-baan koppeling een belangrijke rol, omdat het verantwoordelijk is voor de voorspelling dat gaten een bepaalde orientatie in 2D kwantum putten prefereren. We richten ons op het gedragen van de g-tensor in een roterend magnetisch veld, en onttrekken de anisotropie van de g-tensor. We doen dit door de Zeeman splitsing in een magnetisch veld te bekijken, welke 360 graden over alle 3 vrijheidsgraden kan roteren. Door middel van twee verschillende methodes passen we ons model aan de data en ontrekken vergelijkbare waardes voor de anisotropie voor beide methodes $\left(g_{\|}^{*} \approx 2.2\right.$, $\left.g_{\perp}^{*} \approx 4\right)$. De g-factor loodrecht op het vlak is grofweg twee keer zo groot dan in het vlak. Dit is consistent met de voorspelling dat lichte- en zware-gaten een voorkeur 
hebben voor hoe ze georiënteerd zijn.

In hoofdstuk $\mathbf{0}$ demonstreren we het concept van een enkel-laags gaten kwantum dot welke opereert op basis van depletie. Deze depletie-architectuur heeft verschillende voordelen, zoals het makkelijk toegankelijk zijn voor optische doeleinden en het bezitten van gebieden waar het vrij is van eventuele schade veroorzaakt door elektronenstraal lithografie. De vaste negatieve lading in ALD-gegroeide lagen maakt deze architectuur mogelijk omdat het een 2D gatengas induceert. Gezien de gelijke koppeling van de ladingsovergangen naar naburige elektrodes, kunnen we stellen dat een enkele kwantum dot zich vormt tussen deze elektrodes. Vervolgens kunnen we deze enkele kwantum dot naar een dubbel kwantumdot regime sturen door een interdot-koppelingselektrode te gebruiken. Karakterisatie van de ladingsverschuiving laat een zeer hoge stabiliteit zien, waarbij de stabiliteit een bovengrens van $Q_{0}=0.04 e$ en een ondergrens van $Q_{0}=0.005 e$ heeft. Vergelijking met andere architecturen zoals die gebaseerd op alleen silicium $\left(Q_{0}<0.01 e\right)$ en die op aluminium $\left(Q_{0}<0.15 e\right)$ laat zien dat de resultaten zeer competitief zijn.

Tot slot kunnen we stellen dat gaten een veelbelovende kandidaat zijn voor kwantumcomputatie. Echter voor we zover zijn moeten er nog veel horden worden genomen. Zo hebben we gezien dat het $\mathrm{Si} / \mathrm{SiO}_{2}$ grensvlak veel welhaast intrinsieke defecten bevat. Deze zullen moeten worden weggehaald of vermeden in het streven naar schaling. We hebben laten zien dat dit in ieder geval deels mogelijk is door passivatie, maar een andere mogelijkheid zou zijn door het omschakelen naar een andere isolerende laag. In dit opzicht is het $\mathrm{Si} / \mathrm{SiGe}$ systeem een goede kandidaat. Deze, en zeker vele andere verbeteringen waarvan we slechts een paar hebben aangestipt in dit proefschrift zullen leiden tot het schalen van een echt schaalbare kwantumtechnologie gebaseerd op silicum. 